\title{
IUCrJ
}

Volume 5 (2018)

Supporting information for article:

Do the basic crystal chemistry principles agree with a plethora of recent quantum chemistry data?

Elena Levi, Doron Aurbach and Carlo Gatti 


\title{
Supporting Information
}

\section{Do the Basic Crystal Chemistry Principles Agree with a Plethora of Recent Quantum Chemistry Data?}

\author{
Elena Levi*, Doron Aurbach and Carlo Gatti*
}

\section{Table of Contents}

1. Figure S1. Direct correlation between bond order and the average bond energy for three

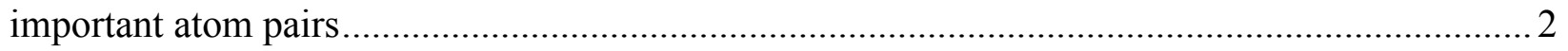

2. Figure S2. Exponential correlations between electron density, $\rho_{c} i j$, and the interatomic distances. The points are quantum chemistry data available in literature.

3. Figure S3. Exponential correlations between effective bond order, $B O_{i j}$, and the interatomic distances. The points are the quantum chemistry data available in literature. The crystal chemistry curves marked in red are presented for comparison. $7-10$

4. Figure S4. Correlation between the $B O$ parameter $R_{0} i j$ and electron density constant $C i j . . \quad 11$

5. Figure S5. Bond order parameter $R_{0} i j$ (a) and electron density constant $C i j$ (b) $v s$. the sum of the covalent radii of $i$ and $\mathrm{j}$ atoms

6. Figure S6. Relationship between electron density and bond order for some atom pairs. 12

7. Figure S7. Additional parameters of the ED topology, which are not related to the Pauling's principles. A - Laplacian of the electron density as a function of the interatomic distance for the $\mathrm{C}-\mathrm{C}, \mathrm{Fe}-\mathrm{C}, \mathrm{Cr}-\mathrm{Cr}$ and $\mathrm{Fe}-\mathrm{O}$ bonds. B - Energy densities of the $\mathrm{Cr}-\mathrm{Cr}$ bonds as a function of their BOs. 12

8. Figure S8. Linear correlation between the BOs of the Mo-O bonds calculated by Eq. 1 and the square of the stretching frequencies of various molybdates obtained in Raman spectroscopy... 13

9. Table S1. The results of the exponential fitting of the quantum chemistry data by Eqs 1 and 3: Bond valence parameters and electron density constants for typical atomic pairs. $n$ is a number of data points. 14-17

10. Table S2. The results of the $B O$ calculations for the $\operatorname{Re}_{6}$-cluster complexes 18

11. Table S3. Bond valence parameters used in the $B O$ calculations for the $\operatorname{Re}_{6}$-cluster complexes... 
Figure S1. Direct correlation between bond order and the average bond energy for three important atom pairs (The data from ref. ${ }^{[1]}$ ).

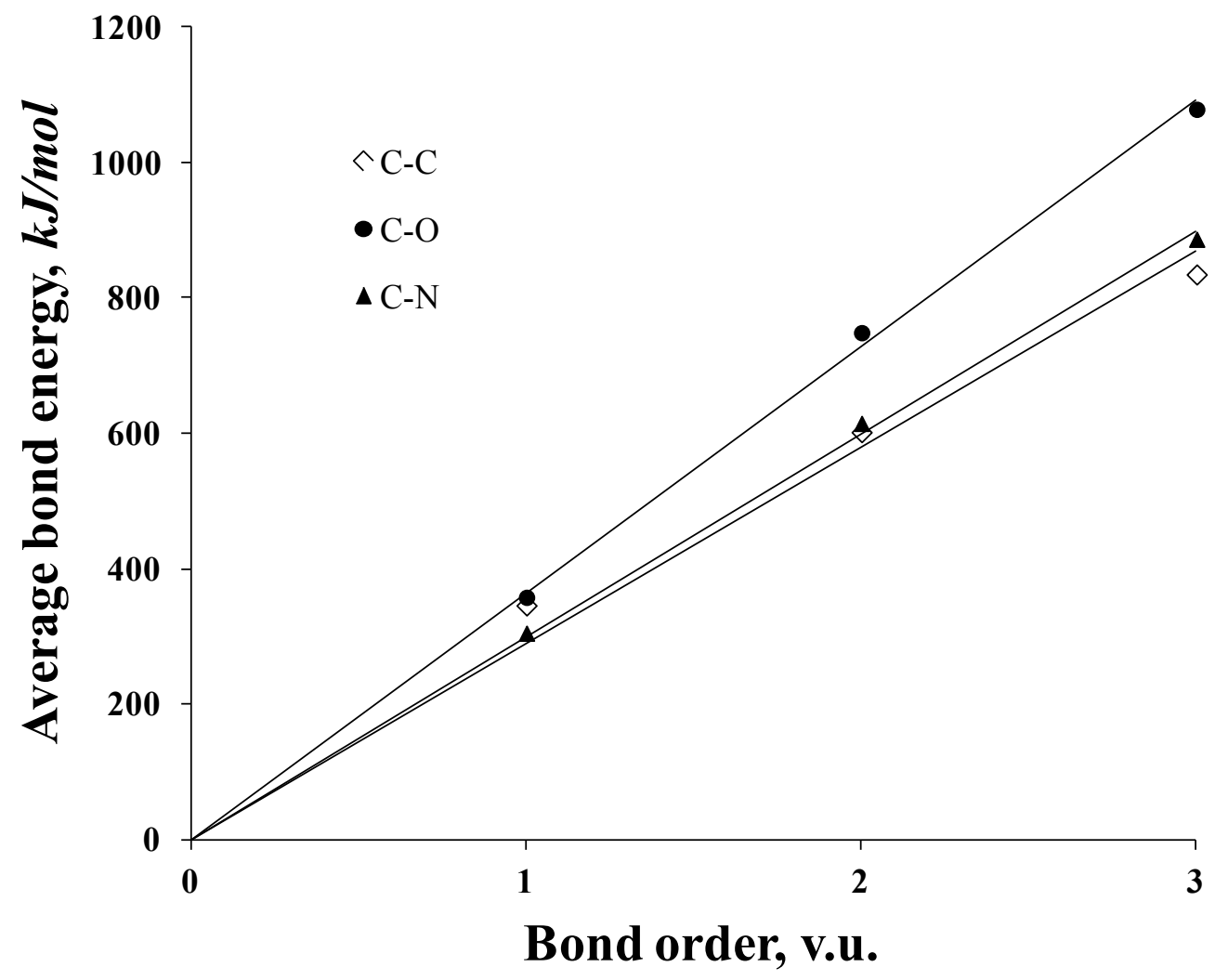


Figure S2. Exponential correlations between electron density, $\rho_{c} i j$, and the interatomic distances. The points are quantum chemistry data available in literature.
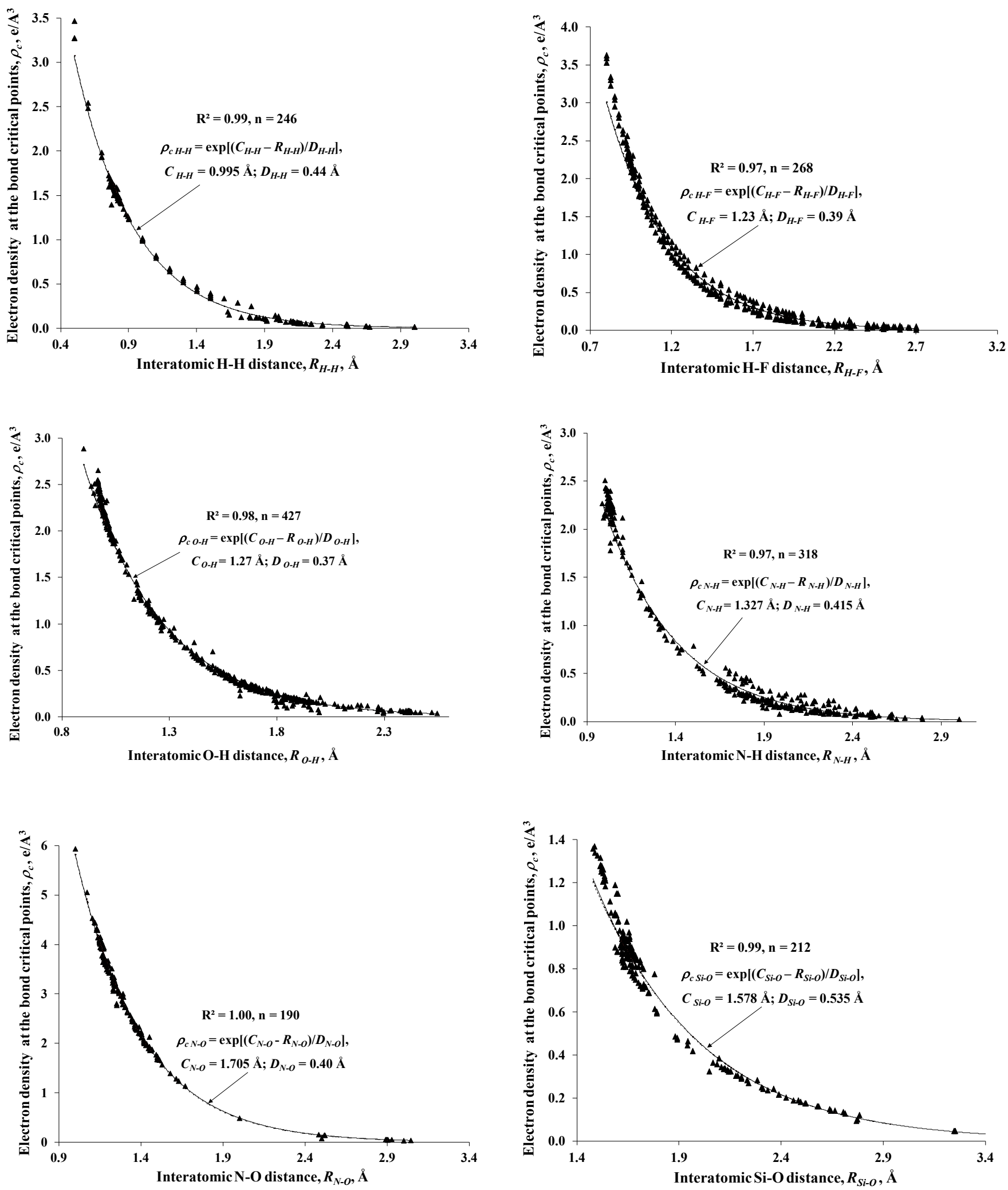

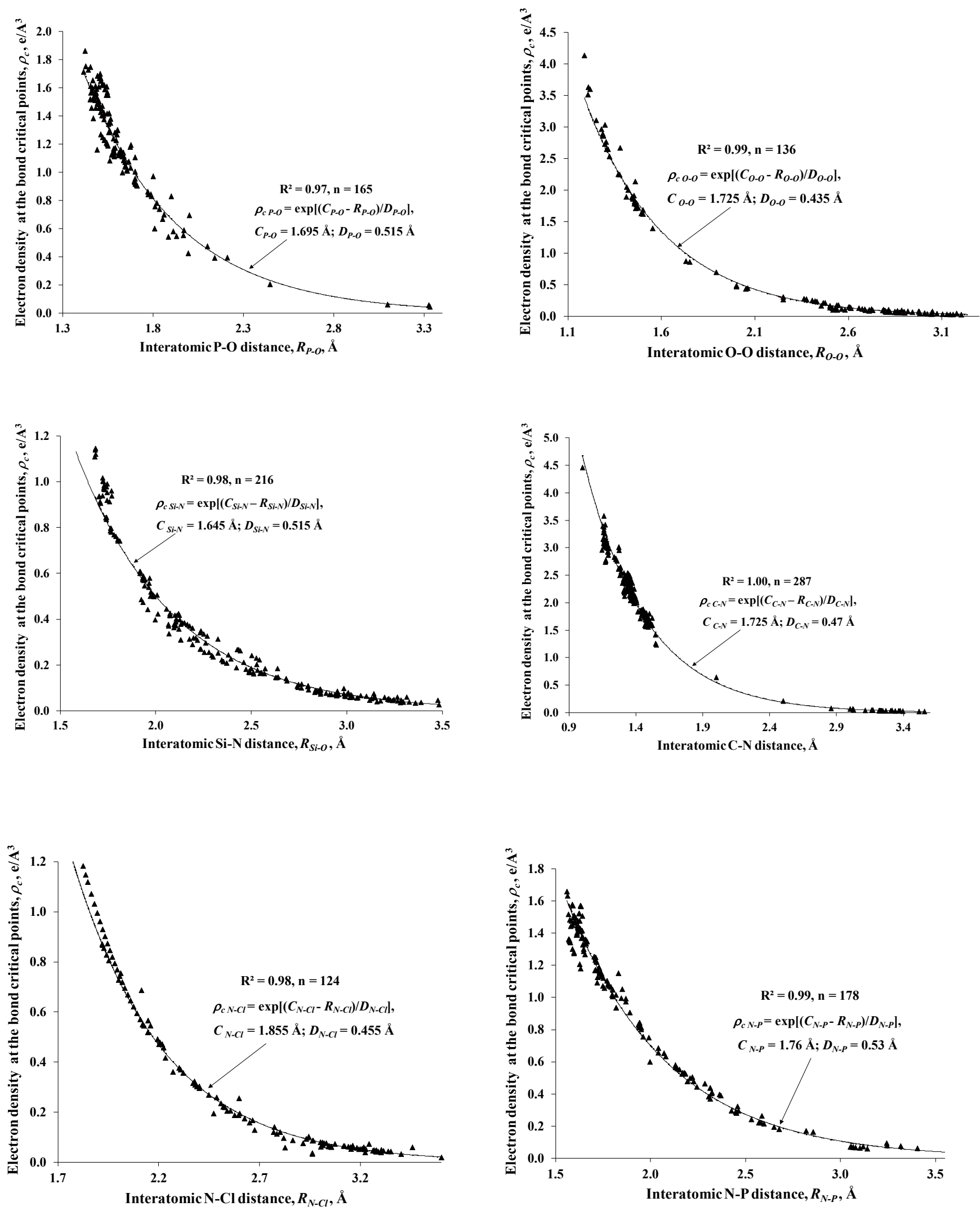

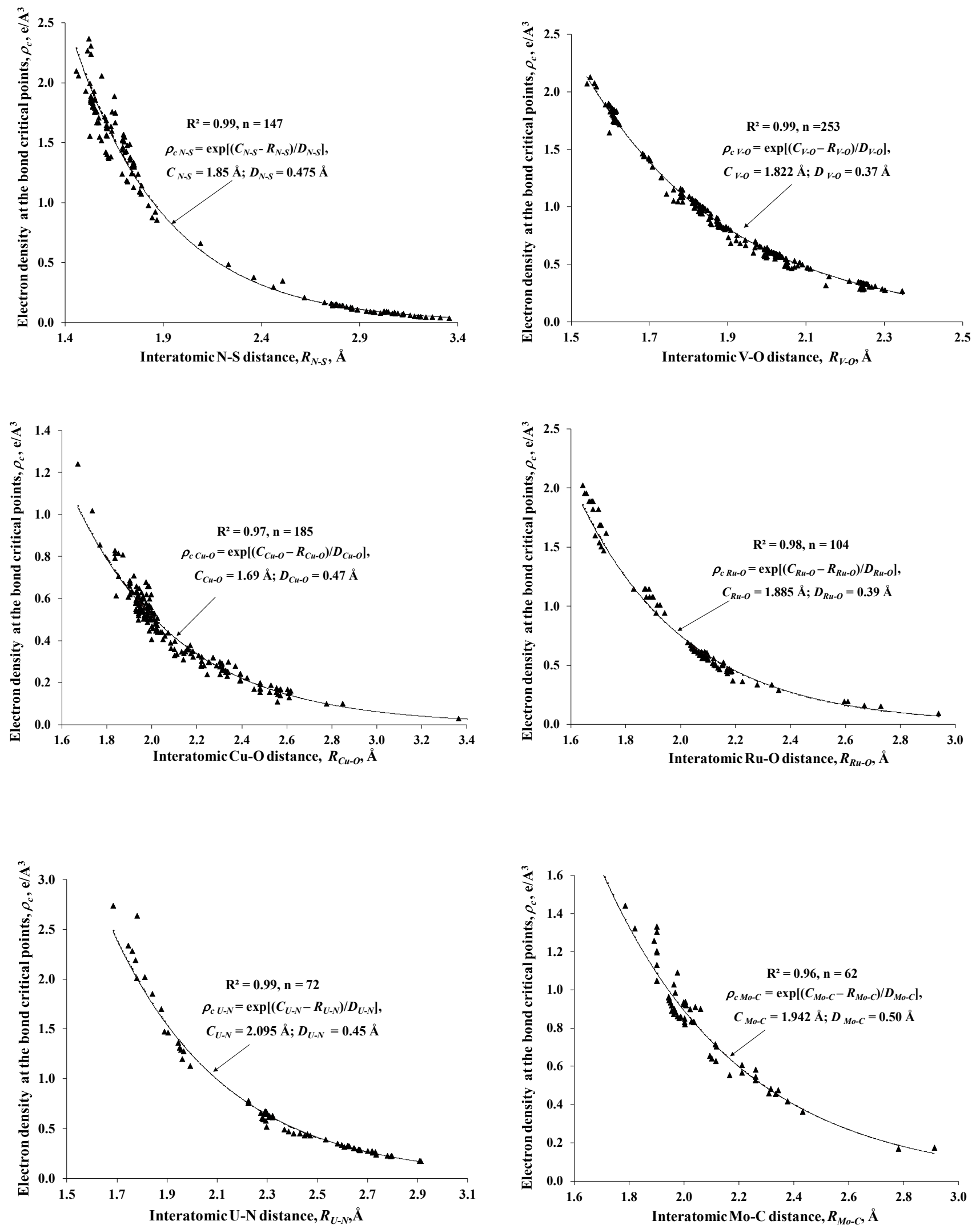

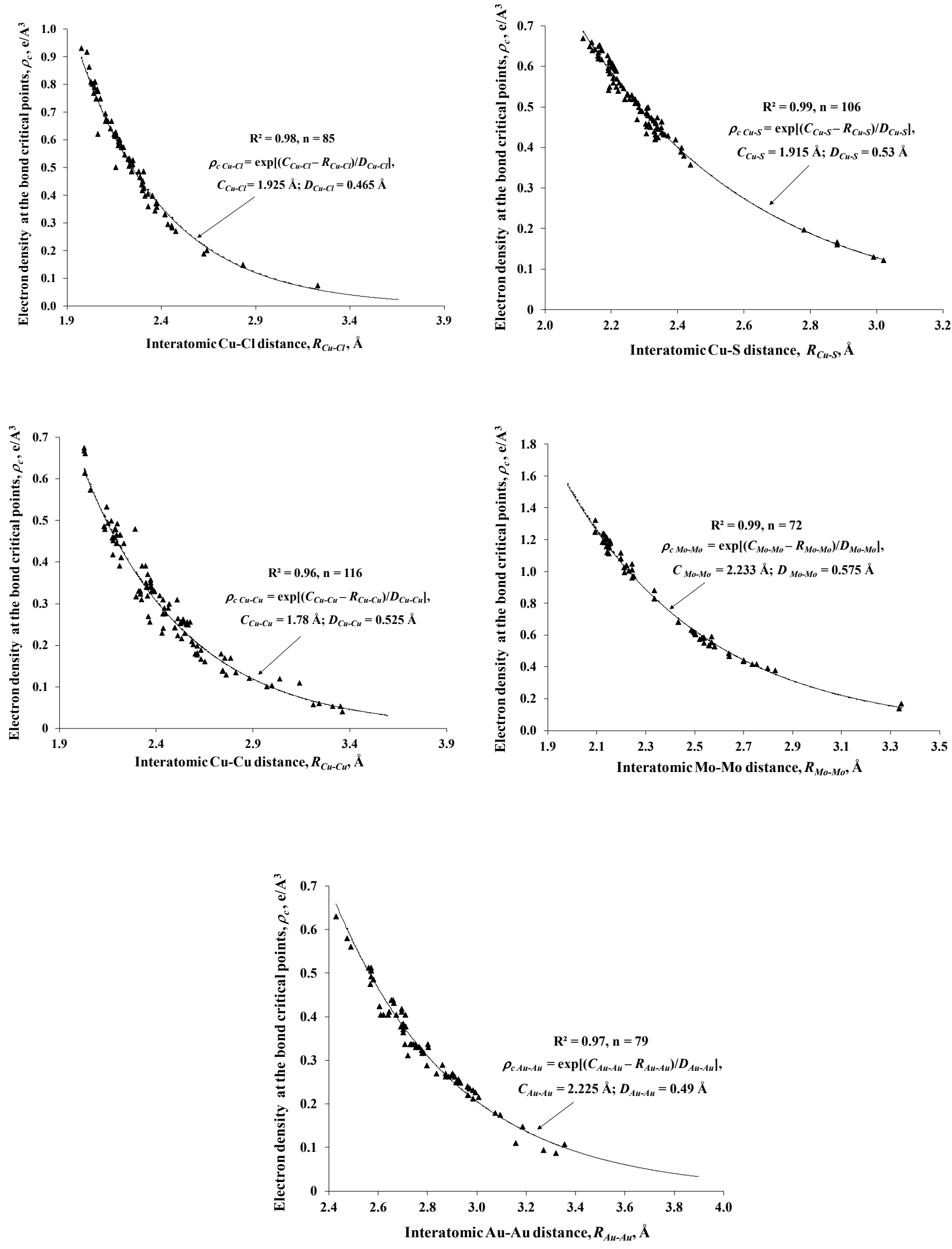
Figure S3. Exponential correlations between effective bond order, $B O_{i j}$, and the interatomic distances. The points are the quantum chemistry data available in literature. The crystal chemistry curves marked in red are presented for comparison.
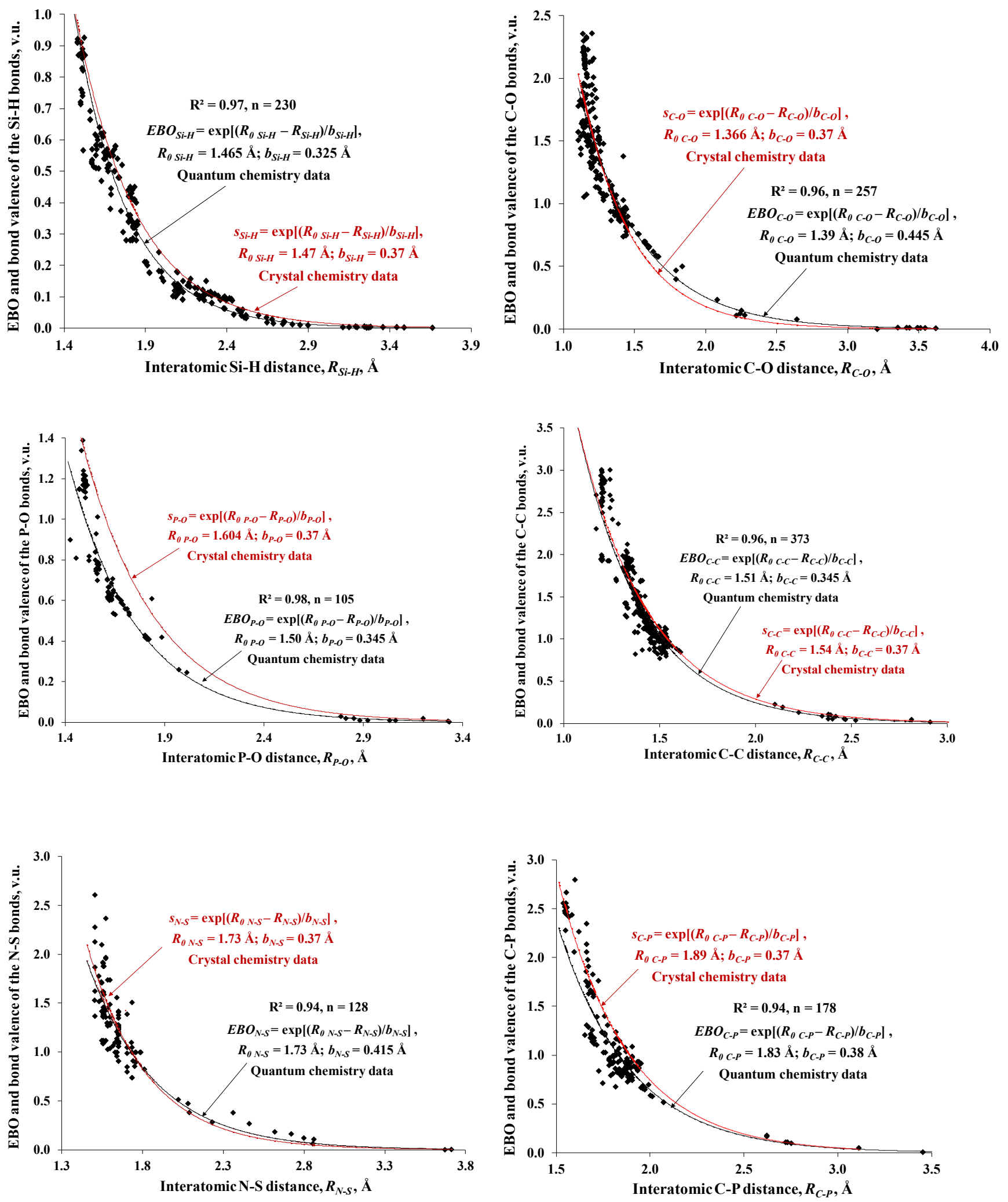

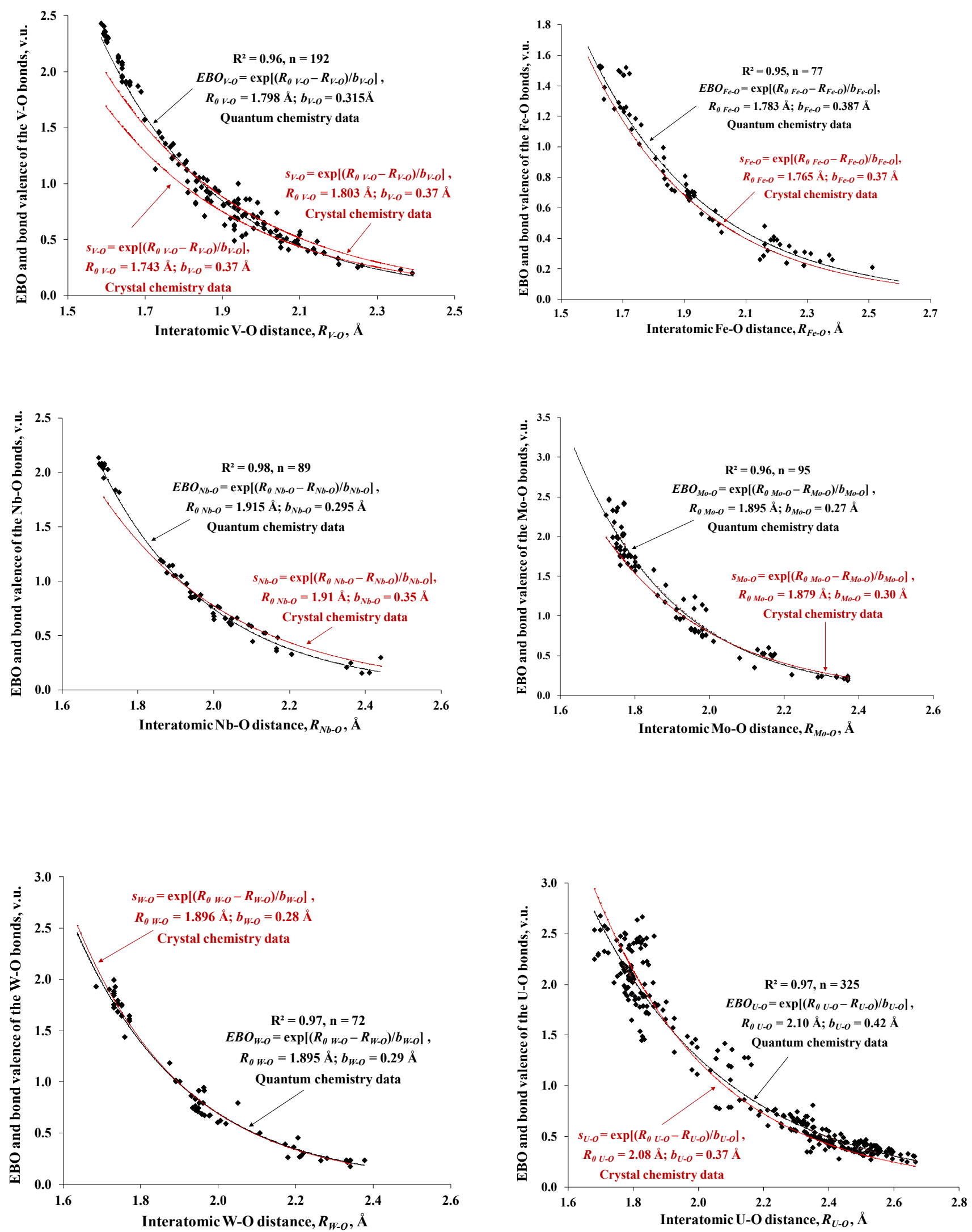

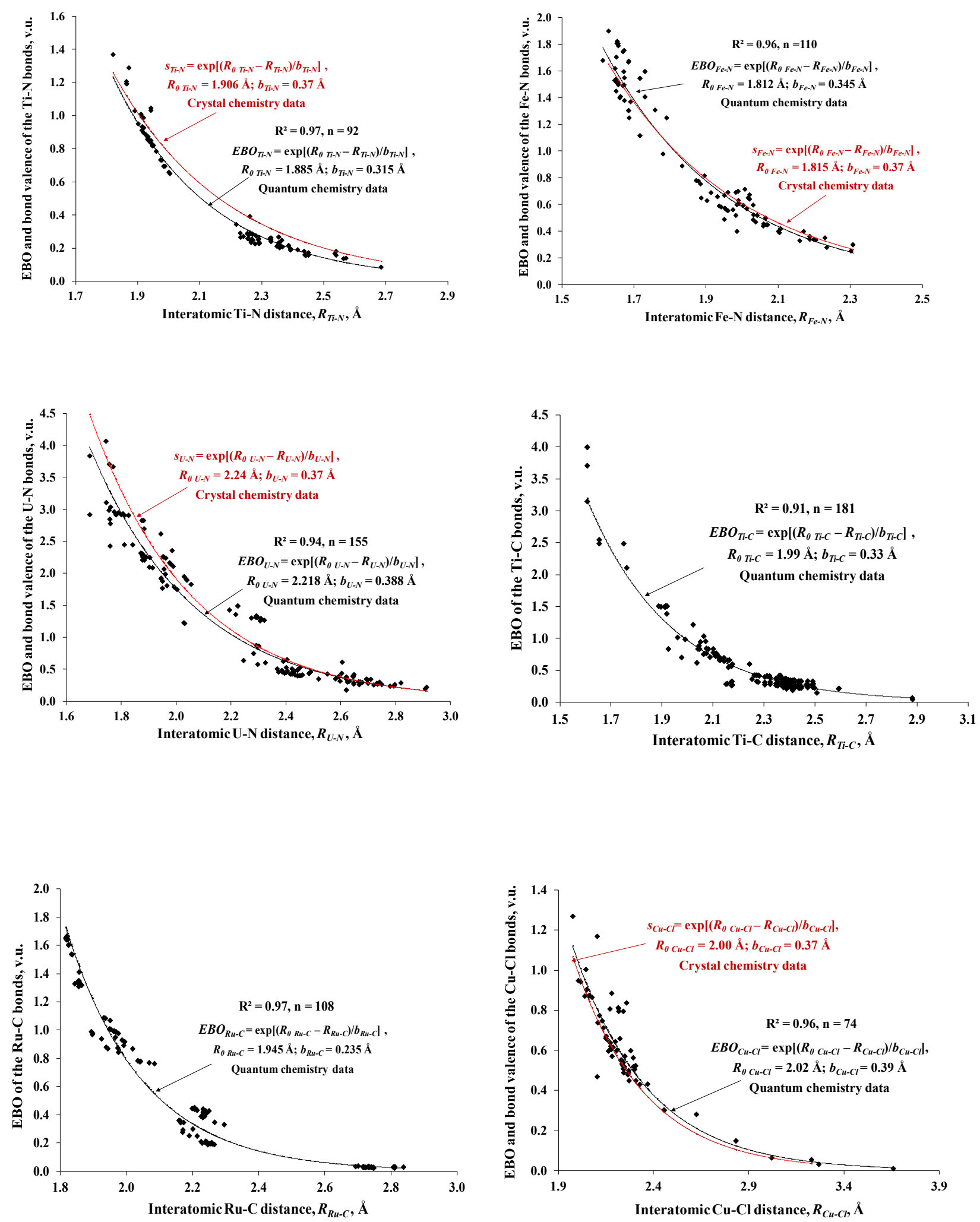

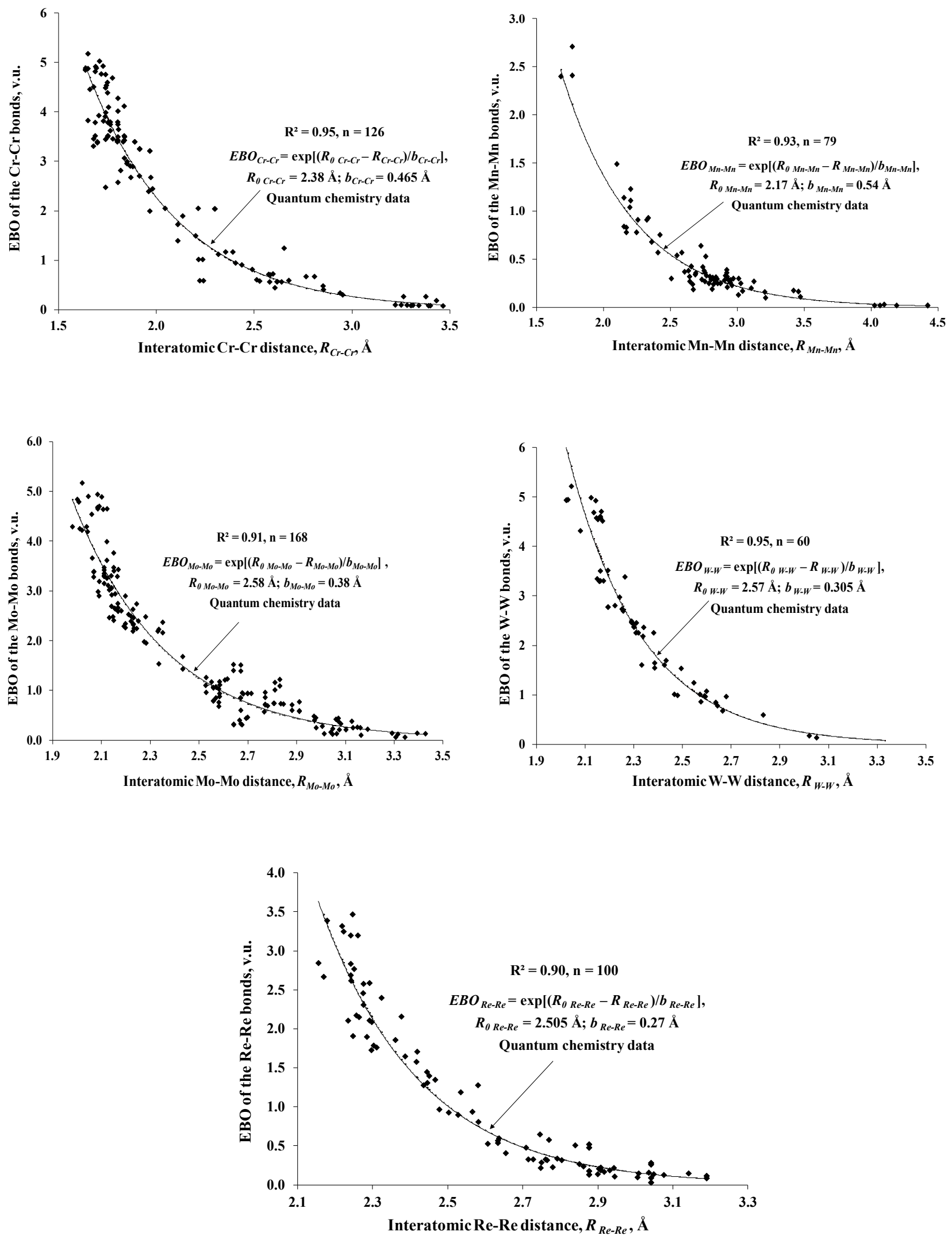
Figure S4. Correlation between the $B O$ parameter $R_{0} i j$ and electron density constant $C i j$.



Figure S5. Bond order parameter $R_{0}(\mathrm{~A})$ and electron density constant $C i j(\mathrm{~B}) v s$. the sum of the covalent radii of $i$ and $\mathrm{j}$ atoms.
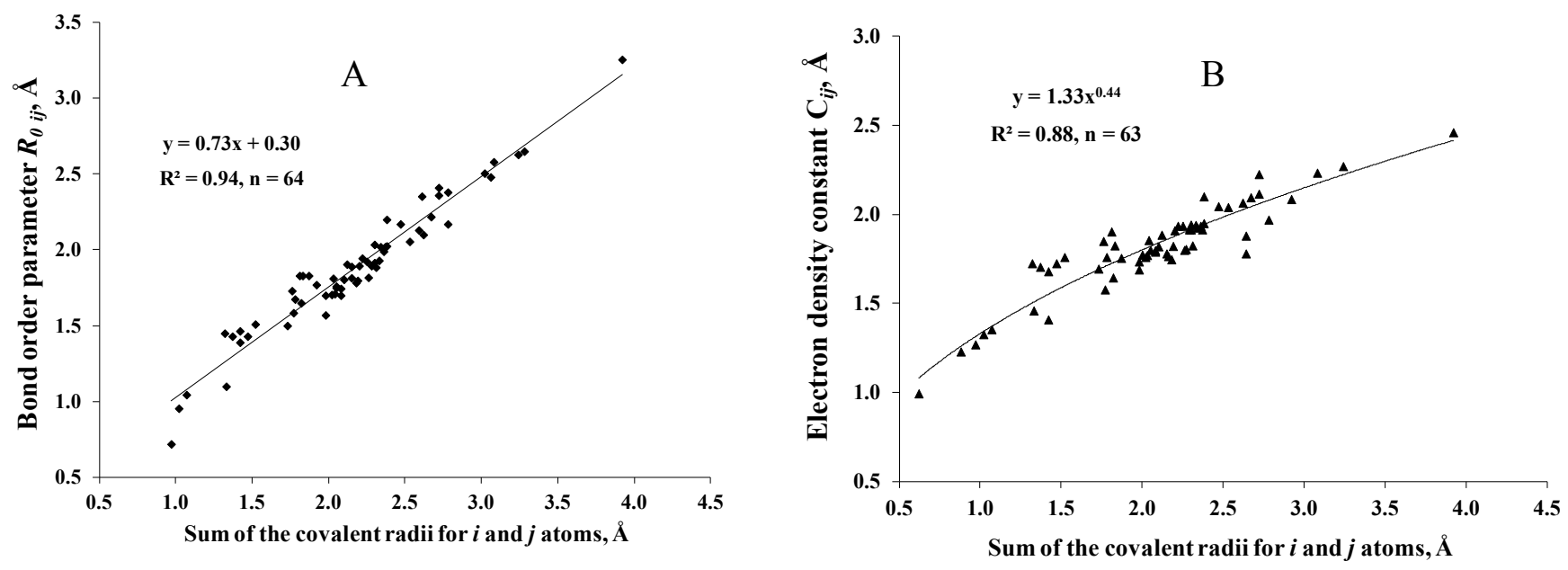
Figure S6. Relationship between electron density and bond order for some atom pairs.

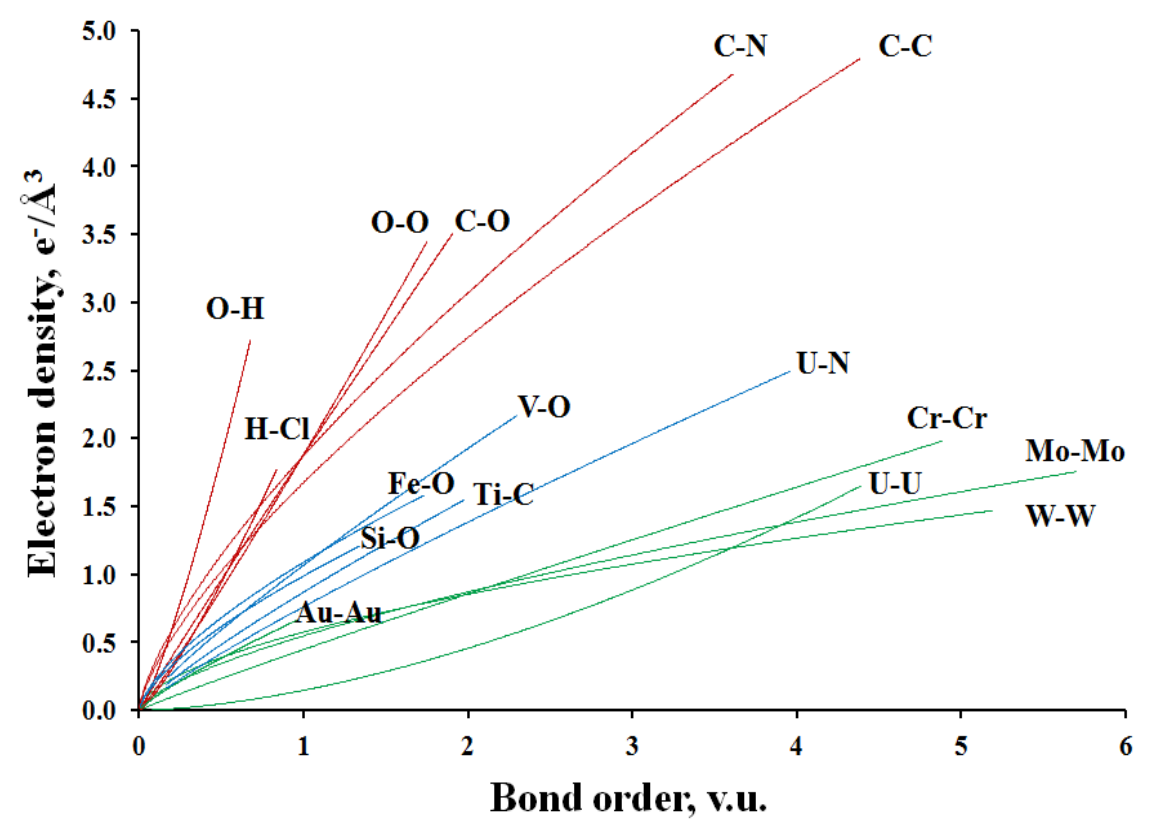

Figure S7. Additional parameters of the ED topology, which are not related to the Pauling's principles. A - Laplacian of the electron density as a function of the interatomic distance for the $\mathrm{C}-\mathrm{C}, \mathrm{Fe}-\mathrm{C}, \mathrm{Cr}-\mathrm{Cr}$ and $\mathrm{Fe}-\mathrm{O}$ bonds. B - Energy densities of the $\mathrm{Cr}-\mathrm{Cr}$ bonds as a function of their BOs.
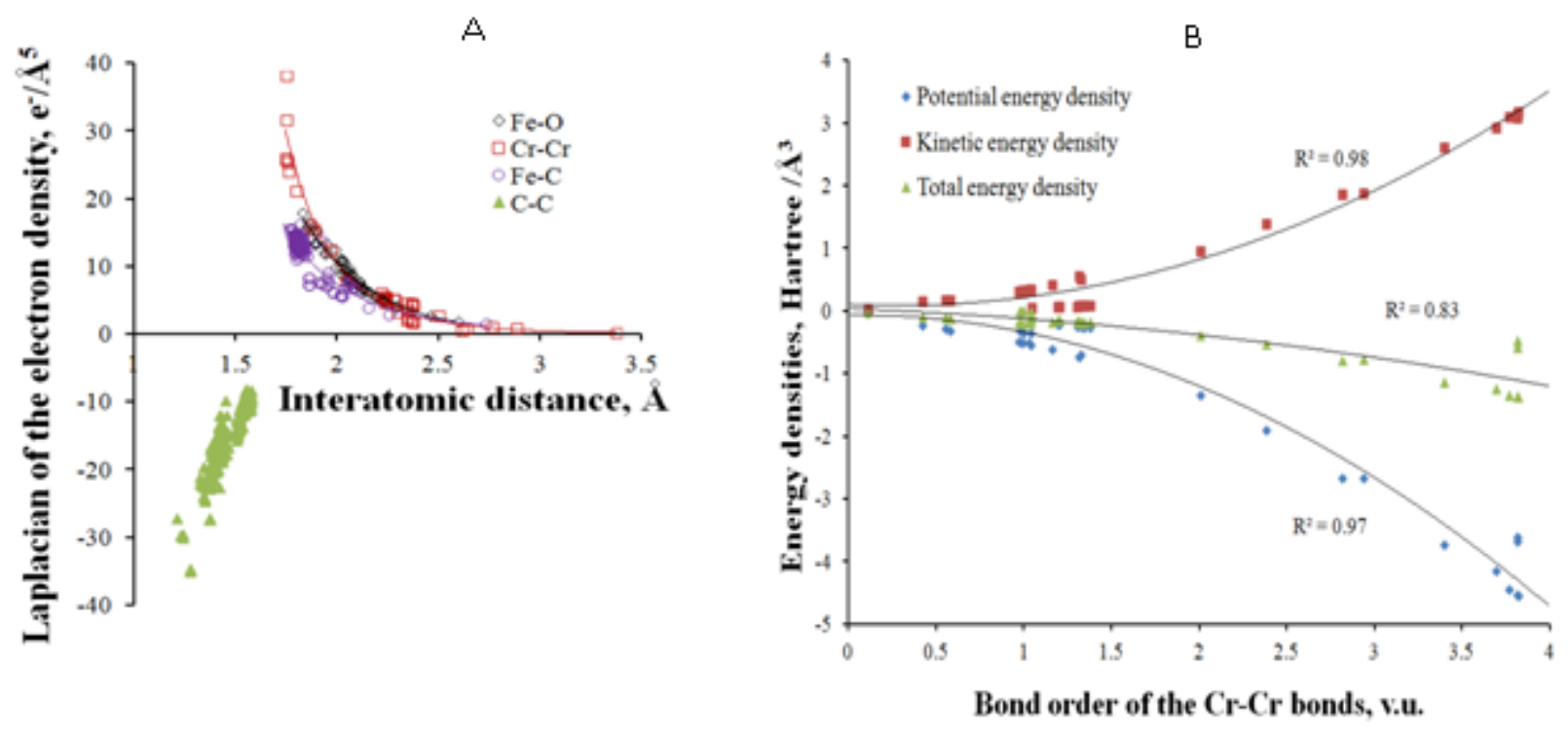

These parameters are widely used to describe the differences in the nature of chemical bonds. For example, as can be seen in Fig. S7A, in contrast to ionic or mixed ionic/covalent interactions, the Laplacian of the pure covalent $\mathrm{C}-\mathrm{C}$ bonds is clearly negative. The key parameter to define the bond character is then the ratio between absolute value of the potential and kinetic energy 
densities at the bond critical point ${ }^{[2]}$, which is in turn related to the Laplacian value at this same position by the local expression of the quantum virial theorem ${ }^{[3]}$. For the ionic bonds this ratio is smaller then 1 (Laplacian $>0$ ), for the pure covalent bonds the ratio is larger than 2 (Laplacian $<0$ ), while the mixed interactions have the ratio between 1 and 2 (Laplacian $>0$ ). According to this parameter, the $\mathrm{Cr}-\mathrm{Cr}$ interactions can be characterized as mixed ionic/covalent ones (Fig. S7B).

Figure S8. Linear correlation between the BOs of the Mo-O bonds calculated by Eq. 1 and the square of the stretching frequencies of various molybdates obtained in Raman spectroscopy. (The interatomic distances and stretching frequencies are taken from ref. ${ }^{[4]}$ )

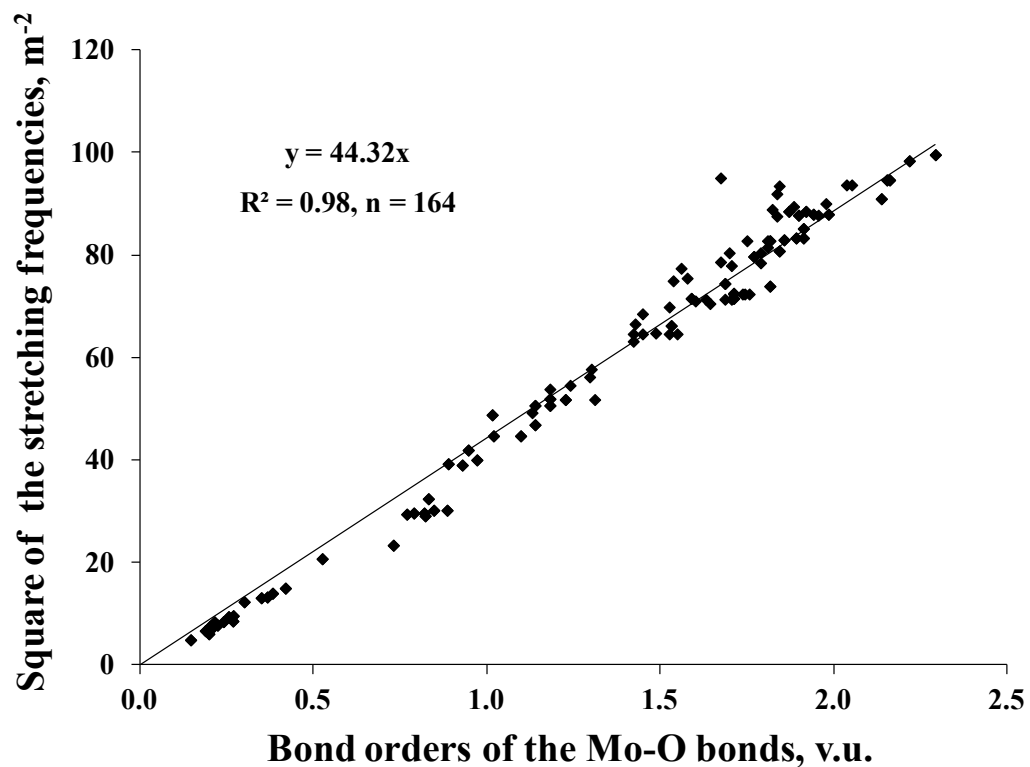


Table S1. The results of the exponential fitting of the quantum chemistry data by Eqs 1 and 3: Bond valence parameters and electron density constants for typical atomic pairs. $n$ is a number of data points.

\begin{tabular}{|c|c|c|c|c|c|c|c|c|c|c|}
\hline \multirow[t]{2}{*}{$\mathrm{N}$} & \multirow[t]{2}{*}{ Bond } & \multicolumn{2}{|c|}{$\begin{array}{c}\text { Bond valence } \\
\text { parameters, } \\
\AA\end{array}$} & \multicolumn{2}{|c|}{$\begin{array}{l}\text { Quality of } \\
\text { exponential } \\
\text { fitting }\end{array}$} & \multicolumn{2}{|c|}{$\begin{array}{c}\text { Electron } \\
\text { density } \\
\text { constants, } \\
\mathrm{e} / \AA^{3}\end{array}$} & \multicolumn{2}{|c|}{$\begin{array}{l}\text { Quality of } \\
\text { exponential } \\
\text { fitting }\end{array}$} & \multirow[t]{2}{*}{ Ref. } \\
\hline & & $R_{0 i j}$ & $b_{i j}$ & $R^{2}$ & $n$ & $C_{i j}$ & $D_{i j}$ & $R^{2}$ & $n$ & \\
\hline 1 & $\mathrm{H}-\mathrm{H}$ & & & & & 0.995 & 0.44 & 0.99 & 246 & [5] \\
\hline 2 & $\mathrm{H}-\mathrm{F}$ & & & & & 1.23 & 0.39 & 0.97 & 268 & {$[2 \mathrm{~b}, 5 \mathrm{~b}, 5 \mathrm{o}, 6]$} \\
\hline 3 & $\mathrm{O}-\mathrm{H}$ & 0.72 & 0.46 & 0.96 & 187 & 1.27 & 0.37 & 0.98 & 427 & {$[5 b, 5 f, 7]$} \\
\hline 4 & $\mathrm{~N}-\mathrm{H}$ & 0.955 & 0.41 & 0.96 & 126 & 1.327 & 0.415 & 0.97 & 318 & $\begin{array}{c}{[5 \mathrm{~b}, 5 \mathrm{e}, 7 \mathrm{a}, 7 \mathrm{e}, 7 \mathrm{~g}, 7 \mathrm{k}, 7 \mathrm{~m}, 7 \mathrm{p}, 7 \mathrm{r}} \\
\mathrm{u}, 7 \mathrm{x}, 7 \mathrm{ae}, 8]\end{array}$ \\
\hline 5 & C-H & 1.045 & 0.365 & 0.98 & 75 & 1.355 & 0.435 & 0.99 & 398 & {$[5 \mathrm{~b}, 5 \mathrm{~d}-\mathrm{f}, 5 \mathrm{o}, 7 \mathrm{~s}, 8 \mathrm{a}, 8 \mathrm{~b}, 8 \mathrm{i}, 9]$} \\
\hline 6 & $\mathrm{Si}-\mathrm{H}$ & 1.465 & 0.325 & 0.97 & 230 & 1.41 & 0.475 & 0.98 & 147 & {$[8 \mathrm{u}, 10]$} \\
\hline 7 & $\mathrm{H}-\mathrm{Cl}$ & 1.10 & 0.56 & 0.93 & 125 & 1.46 & 0.455 & 0.98 & 198 & $\begin{array}{c}{[5 \mathrm{~m}, 6 \mathrm{~b}, 6 \mathrm{~d}, 7 \mathrm{~m}, 7 \mathrm{~s}, 8 \mathrm{n}, 8 \mathrm{p}, 8 \mathrm{~s}} \\
8 \mathrm{u}, 11]\end{array}$ \\
\hline 8 & $\mathrm{C}-\mathrm{O}$ & 1.39 & 0.445 & 0.96 & 257 & 1.68 & 0.46 & 0.99 & 345 & $\begin{array}{c}\text { [5b, 5d, 5f, 7h, 7i, 7s, 8a, 8e, 8i, } \\
\text { 9b, 9e, 9f, 9j, 9m, 12] }\end{array}$ \\
\hline 9 & $\mathrm{~N}-\mathrm{O}$ & 1.43 & 0.37 & 0.86 & 240 & 1.705 & 0.40 & 1.00 & 187 & $\begin{array}{c}{[5 \mathrm{~b}, 7 \mathrm{~g}, 7 \mathrm{w}, 7 \mathrm{z}, 8 \mathrm{j}, 8 \mathrm{u}, 9 \mathrm{~d}, 9 \mathrm{l}, 11 \mathrm{r}} \\
12 \mathrm{z}, 12 \mathrm{ae}, 13]\end{array}$ \\
\hline 10 & $\mathrm{Si}-\mathrm{O}$ & 1.585 & 0.365 & 0.90 & 103 & 1.578 & 0.535 & 0.99 & 212 & $\begin{array}{c}{[6 \mathrm{e}, 8 \mathrm{p}, 8 \mathrm{u}, 10 \mathrm{f}, 10 \mathrm{q}, 10 \mathrm{u}, 10 \mathrm{v}} \\
131,14]\end{array}$ \\
\hline 11 & $\mathrm{P}-\mathrm{O}$ & 1.50 & 0.345 & 0.98 & 105 & 1.695 & 0.515 & 0.98 & 165 & {$[8 \mathrm{j}, 12 \mathrm{~s}, 13 \mathrm{l}, 14 \mathrm{k}, 14 \mathrm{v}, 15]$} \\
\hline 12 & $\mathrm{O}-\mathrm{O}$ & 1.45 & 0.47 & 0.92 & 83 & 1.725 & 0.435 & 0.99 & 136 & {$[5 \mathrm{~b}, 5 \mathrm{~d}, 7 \mathrm{j}, 7 \mathrm{ab}, 13 \mathrm{~m}, 14 \mathrm{~d}, 16]$} \\
\hline 13 & $\mathrm{Si}-\mathrm{N}$ & 1.65 & 0.375 & 0.97 & 170 & 1.645 & 0.515 & 0.98 & 216 & $\begin{array}{c}{[8 \mathrm{q}, 10 \mathrm{q}, 10 \mathrm{t}, 10 \mathrm{u}, 14 \mathrm{e}, 14 \mathrm{i}, 14 \mathrm{j},} \\
14 \mathrm{o}, 14 \mathrm{u}, 14 \mathrm{w}, 14 \mathrm{y}, 17]\end{array}$ \\
\hline 14 & $\mathrm{~N}-\mathrm{C}$ & 1.43 & 0.335 & 0.97 & 200 & 1.725 & 0.47 & 1.00 & 287 & $\begin{array}{l}\text { [5b, 5d, 7g-i, 7s, 8a-g, 8i, 8j, 9b, } \\
\text { 9d, 9i, 91, 9o, 12k, 12n, 12r, 12t, } \\
\text { 12aj, 12am, 13e, 13k, 13o-q, 16n, } \\
\text { 17f, 171, 18] }\end{array}$ \\
\hline 15 & $\mathrm{C}-\mathrm{C}$ & 1.51 & 0.345 & 0.96 & 373 & 1.76 & 0.485 & 0.99 & 786 & $\begin{array}{l}{[5 \mathrm{~b}-\mathrm{d}, 5 \mathrm{f}, 7 \mathrm{~h}, 7 \mathrm{i}, 7 \mathrm{p}, 7 \mathrm{~s}, 8 \mathrm{a}, 8 \mathrm{e}, 8 \mathrm{i}} \\
9 \mathrm{~d}-\mathrm{g}, 9 \mathrm{i}-\mathrm{m}, 12 \mathrm{~d}, 12 \mathrm{j}, 12 \mathrm{k}, 12 \mathrm{n}, \\
12 \mathrm{r}, 12 \mathrm{t}, 12 \mathrm{al}, 18 \mathrm{c}, 18 \mathrm{e}-\mathrm{g}, 18 \mathrm{x}, \\
19]\end{array}$ \\
\hline 16 & $\mathrm{Si}-\mathrm{C}$ & 1.83 & 0.37 & 0.90 & 263 & 1.755 & 0.495 & 0.96 & 185 & $\begin{array}{l}\text { [10f, 10i, 10o, 10p, 10u-w, 10z, } \\
\text { 14a, 14f, 14i, 14j, 17j, 17p, 17t, }\end{array}$ \\
\hline
\end{tabular}




\begin{tabular}{|c|c|c|c|c|c|c|c|c|c|c|}
\hline & & & & & & & & & & $18 \mathrm{p}, 19 \mathrm{o}, 20]$ \\
\hline 17 & $\mathrm{~N}-\mathrm{Cl}$ & 1.71 & 0.585 & 0.93 & 66 & 1.855 & 0.455 & 0.98 & 124 & {$[11 \mathrm{~d}, 11 \mathrm{n}, 11 \mathrm{r}, 13 \mathrm{~m}, 21]$} \\
\hline 18 & N-P & 1.675 & 0.335 & 0.95 & 157 & 1.76 & 0.53 & 0.99 & 178 & $\begin{array}{c}{[8 \mathrm{k}, 8 \mathrm{o}, 12 \mathrm{aj}, 14 \mathrm{i}, 15 \mathrm{c}, 15 \mathrm{e}, 15 \mathrm{l}} \\
15 \mathrm{p}, 17 \mathrm{~g}, 17 \mathrm{~m}, 18 \mathrm{~m}, 18 \mathrm{t}, 22]\end{array}$ \\
\hline 19 & $\mathrm{~N}-\mathrm{S}$ & 1.73 & 0.415 & 0.94 & 128 & 1.85 & 0.475 & 0.99 & 147 & {$[5 \mathrm{~d}, 8 \mathrm{~b}, 8 \mathrm{j}, 14 \mathrm{i}, 15 \mathrm{e}, 16 \mathrm{j}, 23]$} \\
\hline 20 & C-P & 1.83 & 0.38 & 0.94 & 178 & 1.825 & 0.58 & 0.94 & 225 & $\begin{array}{c}8 \mathrm{j}, 8 \mathrm{k}, 9 \mathrm{~g}, 9 \mathrm{i}, 9 \mathrm{~m}, 9 \mathrm{o}, 9 \mathrm{q}, 12 \mathrm{n}, \\
12 \mathrm{o}, 15 \mathrm{l}-\mathrm{o}, 18 \mathrm{j}, 18 \mathrm{~m}, 18 \mathrm{t}, 18 \mathrm{v} \\
20 \mathrm{ac}, 22 \mathrm{o}, 22 \mathrm{r}, 24]\end{array}$ \\
\hline 21 & $\mathrm{C}-\mathrm{S}$ & 1.83 & 0.325 & 0.97 & 203 & 1.903 & 0.533 & 0.98 & 220 & $\begin{array}{c}{[5 \mathrm{~d}, 5 \mathrm{~g}, 7 \mathrm{~g}, 7 \mathrm{~h}, 8 \mathrm{~b}, 8 \mathrm{u}, 12 \mathrm{n}, 12 \mathrm{ac}} \\
12 \mathrm{al}, 14 \mathrm{k}, 18 \mathrm{~g}, 18 \mathrm{i}, 18 \mathrm{l}, 18 \mathrm{~s}, 18 \mathrm{v} \\
19 \mathrm{~m}, 23 \mathrm{k}, 25]\end{array}$ \\
\hline 22 & Ti-O & 1.818 & 0.29 & 0.93 & 141 & 1.80 & 0.385 & 0.95 & 51 & {$[9 \mathrm{~g}, 11 \mathrm{f}, 12 \mathrm{~g}, 12 \mathrm{~s}, 16 \mathrm{p}, 26]$} \\
\hline 23 & $\mathrm{~V}-\mathrm{O}$ & 1.798 & 0.315 & 0.96 & 192 & 1.822 & 0.37 & 0.99 & 253 & $\begin{array}{c}{[7 \mathrm{i}, 7 \mathrm{w}, 12 \mathrm{ak}, 13 \mathrm{~b}, 16 \mathrm{e}, 16 \mathrm{p}, 23 \mathrm{~d},} \\
26 \mathrm{k}, 27]\end{array}$ \\
\hline 24 & $\mathrm{Cr}-\mathrm{O}$ & 1.76 & 0.315 & 0.93 & 164 & 1.80 & 0.38 & 0.97 & 61 & {$[16 \mathrm{p}, 26 \mathrm{~h}, 26 \mathrm{j}, 26 \mathrm{k}, 28]$} \\
\hline 25 & $\mathrm{Mn}-\mathrm{O}$ & 1.75 & 0.34 & 0.95 & 53 & 1.805 & 0.38 & 0.99 & 71 & {$[12 \mathrm{t}, 16 \mathrm{p}, 26 \mathrm{~h}, 26 \mathrm{k}, 29]$} \\
\hline 26 & $\mathrm{Fe}-\mathrm{O}$ & 1.783 & 0.387 & 0.95 & 77 & 1.747 & 0.41 & 0.94 & 156 & $\begin{array}{c}12 \mathrm{~m}, 16 \mathrm{p}, 26 \mathrm{~h}, 28 \mathrm{i}, 28 \mathrm{l}, 29 \mathrm{~b}, 29 \mathrm{f}, \\
29 \mathrm{n}, 30]\end{array}$ \\
\hline 27 & $\mathrm{Co}-\mathrm{O}$ & 1.77 & 0.315 & 0.97 & 42 & 1.765 & 0.385 & 0.96 & 99 & $\begin{array}{c}{[7 \mathrm{u}, 13 \mathrm{w}, 16 \mathrm{p}, 26 \mathrm{k}, 29 \mathrm{c}, 29 \mathrm{f}, 30 \mathrm{i},} \\
30 \mathrm{n}, 31]\end{array}$ \\
\hline 28 & $\mathrm{Cu}-\mathrm{O}$ & 1.57 & 0.47 & 0.87 & 149 & 1.69 & 0.47 & 0.97 & 185 & $\begin{array}{c}\text { [7u, 9d, 12t, 16a, 16b, 16p, 26k, } \\
27 \mathrm{~g}, 29 \mathrm{~b}, 29 \mathrm{~h}, 31 \mathrm{c}, 32]\end{array}$ \\
\hline 29 & $\mathrm{Nb}-\mathrm{O}$ & 1.915 & 0.295 & 0.98 & 89 & 1.915 & 0.39 & 0.95 & 34 & {$[191,26 \mathrm{k}, 27 \mathrm{a}, 27 \mathrm{~h}, 33]$} \\
\hline 30 & Mo-O & 1.895 & 0.27 & 0.96 & 95 & 1.91 & 0.37 & 0.99 & 58 & {$[12 \mathrm{x}, 15 \mathrm{a}, 26 \mathrm{k}, 27 \mathrm{a}, 28 \mathrm{~h}, 33 \mathrm{c}, 34]$} \\
\hline 31 & $\mathrm{Ru}-\mathrm{O}$ & 1.905 & 0.355 & 0.95 & 46 & 1.885 & 0.39 & 0.98 & 104 & [26k, 30k, 34c, 35] \\
\hline 32 & $\mathrm{~W}-\mathrm{O}$ & 1.895 & 0.29 & 0.97 & 72 & & & & & $\begin{array}{c}{[26 \mathrm{j}, 26 \mathrm{k}, 27 \mathrm{a}, 28 \mathrm{l}, 33 \mathrm{c}, 34 \mathrm{~h}, 34 \mathrm{~m},} \\
36]\end{array}$ \\
\hline 33 & U-O & 2.10 & 0.42 & 0.97 & 325 & 2.065 & 0.42 & 0.97 & 151 & {$[8 \mathrm{~b}, 25 \mathrm{e}, 261,27 \mathrm{a}, 37]$} \\
\hline 34 & $\mathrm{Pu}-\mathrm{O}$ & 2.055 & 0.385 & 0.98 & 38 & 2.04 & 0.39 & 0.99 & 34 & {$[7 \mathrm{f}, 37 \mathrm{~m}, 37 \mathrm{q}, 37 \mathrm{v}, 37 \mathrm{aa}, 38]$} \\
\hline 35 & Ti-N & 1.885 & 0.315 & 0.97 & 92 & 1.825 & 0.47 & 0.98 & 86 & [26i-k, 39] \\
\hline 36 & $\mathrm{Cr}-\mathrm{N}$ & 1.805 & 0.285 & 0.92 & 109 & 1.82 & 0.42 & 0.97 & 93 & {$[26 \mathrm{j}, 26 \mathrm{k}, 28 \mathrm{e}, 28 \mathrm{~g}, 28 \mathrm{l}, 40]$} \\
\hline 37 & $\mathrm{Fe}-\mathrm{N}$ & 1.812 & 0.345 & 0.96 & 110 & 1.771 & 0.45 & 0.96 & 83 & $\begin{array}{c}{[13 \mathrm{c}, 13 \mathrm{~d}, 13 \mathrm{u}, 26 \mathrm{k}, 30 \mathrm{~d}, 30 \mathrm{~g}, 30 \mathrm{i}} \\
30 \mathrm{k}, 30 \mathrm{o}, 30 \mathrm{p}, 41]\end{array}$ \\
\hline
\end{tabular}




\begin{tabular}{|c|c|c|c|c|c|c|c|c|c|c|}
\hline 38 & Co-N & & & & & 1.76 & 0.46 & 0.95 & 139 & $\begin{array}{c}{[12 \mathrm{j}, 19 \mathrm{k}, 26 \mathrm{k}, 281,30 \mathrm{i}, 31 \mathrm{~b}-\mathrm{e},} \\
31 \mathrm{i}-1,40 \mathrm{f}, 41 \mathrm{n}, 42]\end{array}$ \\
\hline 39 & $\mathrm{Cu}-\mathrm{N}$ & 1.70 & 0.395 & 0.94 & 134 & 1.735 & 0.475 & 0.91 & 158 & $\begin{array}{c}{[9 \mathrm{~d}, 12 \mathrm{t}, 18 \mathrm{c}, 18 \mathrm{~h}, 29 \mathrm{~h}, 31 \mathrm{c}, 32 \mathrm{~g},} \\
32 \mathrm{~h}, 32 \mathrm{~m}, 32 \mathrm{r}-\mathrm{u}, 32 \mathrm{z}, 32 \mathrm{ac}-\mathrm{ah}, \\
\text { 32ak-am, 34c, 40f, 41n, 43] }\end{array}$ \\
\hline 40 & Mo-N & 1.923 & 0.325 & 0.92 & 116 & 1.935 & 0.43 & 0.99 & 69 & $\begin{array}{c}{[12 \mathrm{x}, 12 \mathrm{z}, 19 \mathrm{c}, 26 \mathrm{j}, 26 \mathrm{k}, 34 \mathrm{c}, 34 \mathrm{~g}} \\
34 \mathrm{l}, 36 \mathrm{a}, 44]\end{array}$ \\
\hline 41 & $\mathrm{~W}-\mathrm{N}$ & 1.93 & 0.275 & 0.95 & 140 & 1.94 & 0.43 & 0.98 & 53 & {$[12 \mathrm{ag}, 18 \mathrm{~b}, 23 \mathrm{f}, 26 \mathrm{k}, 36 \mathrm{a}, 44 \mathrm{f}, 45]$} \\
\hline 42 & U-N & 2.218 & 0.388 & 0.94 & 155 & 2.095 & 0.45 & 0.99 & 72 & $\begin{array}{c}{[8 \mathrm{~b}, 8 \mathrm{~g}, 37 \mathrm{f}, 37 \mathrm{~h}, 37 \mathrm{n}-\mathrm{q}, 37 \mathrm{w}} \\
37 \mathrm{x}, 37 \mathrm{ac}, 37 \mathrm{ad}, 46]\end{array}$ \\
\hline 43 & Ti-C & 1.99 & 0.33 & 0.91 & 181 & 1.935 & 0.39 & 0.95 & 96 & \begin{tabular}{|}
$9 \mathrm{a}, 9 \mathrm{~g}, 9 \mathrm{~h}, 11 \mathrm{f}, 12 \mathrm{~g}, 19 \mathrm{i}, 19 \mathrm{p}$ \\
$26 \mathrm{e}, 26 \mathrm{i}, 26 \mathrm{j}, 37 \mathrm{n}, 39 \mathrm{c}, 39 \mathrm{e}, 47]$
\end{tabular} \\
\hline 44 & $\mathrm{Cr}-\mathrm{C}$ & 1.89 & 0.345 & 0.90 & 157 & & & & & $\begin{array}{c}9 \mathrm{a}, 12 \mathrm{~d}, 12 \mathrm{f}, 12 \mathrm{j}, 12 \mathrm{w}, 12 \mathrm{aa}, \\
12 \mathrm{ah}, 19 \mathrm{e}, 19 \mathrm{f}, 26 \mathrm{j}, 28 \mathrm{~g}, 40 \mathrm{e}, 47 \mathrm{a}, \\
48]\end{array}$ \\
\hline 45 & $\mathrm{Mn}-\mathrm{C}$ & 1.815 & 0.315 & 0.95 & 47 & 1.78 & 0.485 & 0.94 & 126 & $\begin{array}{c}\text { [9j, 9k, 9m, 12i, 12u, 12x, 19f, } \\
191,47 \mathrm{a}, 49]\end{array}$ \\
\hline 46 & $\mathrm{Fe}-\mathrm{C}$ & 1.745 & 0.36 & 0.90 & 146 & 1.79 & 0.53 & 0.95 & 188 & $\begin{array}{c}\text { [9c, 9k, 12d, 12f, 12h, 12i, 12m, } \\
\text { 12aa, 19f, 22b, 41i, 47a, 47b, 47g, } \\
47 \mathrm{j}, 48 \mathrm{f}, 50]\end{array}$ \\
\hline 47 & $\mathrm{Co}-\mathrm{C}$ & 1.705 & 0.46 & 0.89 & 70 & & & & & $\begin{array}{c}{[9 \mathrm{f}, 12 \mathrm{c}, 12 \mathrm{~d}, 31 \mathrm{e}, 42 \mathrm{i}, 47 \mathrm{a}, 47 \mathrm{i}} \\
47 \mathrm{j}, 49 \mathrm{~d}, 50 \mathrm{~g}, 51]\end{array}$ \\
\hline 48 & $\mathrm{Ni}-\mathrm{C}$ & & & & & 1.773 & 0.48 & 0.97 & 77 & [10i, 12aa, 48f, 52] \\
\hline 49 & $\mathrm{Cu}-\mathrm{C}$ & 1.70 & 0.347 & 0.82 & 122 & 1.798 & 0.46 & 0.98 & 42 & $\begin{array}{c}{[12 \mathrm{~d}, 18 \mathrm{c}, 18 \mathrm{~d}, 32 \mathrm{ac}, 43 \mathrm{~b}, 43 \mathrm{~d},} \\
43 \mathrm{~h}, 43 \mathrm{~m}, 48 \mathrm{i}, 49 \mathrm{~d}, 53]\end{array}$ \\
\hline 50 & Mo-C & 2.035 & 0.365 & 0.94 & 109 & 1.942 & 0.50 & 0.96 & 62 & $\begin{array}{c}{[12 \mathrm{v}-\mathrm{z}, 12 \mathrm{ah}, 19 \mathrm{c}, 26 \mathrm{j}, 34 \mathrm{~b}, 44 \mathrm{e},} \\
47 \mathrm{~g}, 48 \mathrm{~g}, 54]\end{array}$ \\
\hline 51 & $\mathrm{Ru}-\mathrm{C}$ & 1.945 & 0.235 & 0.97 & 108 & 1.935 & 0.45 & 0.93 & 95 & {$[12 \mathrm{ab}, 22 \mathrm{~b}, 35 \mathrm{c}, 49 \mathrm{e}, 55]$} \\
\hline 52 & $\mathrm{~W}-\mathrm{C}$ & 2.025 & 0.29 & 0.91 & 230 & 1.95 & 0.465 & 0.92 & 85 & $\begin{array}{l}12 \mathrm{ag}, 12 \mathrm{ah}, 26 \mathrm{j}, 28 \mathrm{~g}, 36 \mathrm{~b}, 45 \mathrm{c} \\
48 \mathrm{~g}, 50 \mathrm{c}, 54 \mathrm{f}, 541,54 \mathrm{~m}, 55 \mathrm{f}, 56]\end{array}$ \\
\hline 53 & $\mathrm{U}-\mathrm{C}$ & 2.36 & 0.475 & 0.91 & 162 & 2.115 & 0.46 & 0.88 & 52 & $\begin{array}{c}{[37 \mathrm{c}, 37 \mathrm{n}, 46 \mathrm{~g}, 46 \mathrm{~h}, 46 \mathrm{k}, 46 \mathrm{q}} \\
47 \mathrm{c}, 54 \mathrm{~b}, 57]\end{array}$ \\
\hline 54 & $\mathrm{Cu}-\mathrm{Cl}$ & 2.02 & 0.39 & 0.96 & 74 & 1.925 & 0.465 & 0.98 & 85 & [32ag, 43f, 43h, 43k, 53j, 58] \\
\hline 55 & $\mathrm{Ag}-\mathrm{Cl}$ & 2.17 & 0.43 & 0.96 & 41 & 2.045 & 0.475 & 0.99 & 41 & {$[53 \mathrm{j}, 58 \mathrm{~b}, 58 \mathrm{~d}]$} \\
\hline 56 & $\mathrm{Au}-\mathrm{Cl}$ & 2.20 & 0.42 & 0.97 & 55 & 2.10 & 0.52 & 1.00 & 53 & {$[11 \mathrm{q}, 53 \mathrm{j}, 58 \mathrm{~b}, 58 \mathrm{~d}, 58 \mathrm{e}, 59]$} \\
\hline 57 & $\mathrm{Fe}-\mathrm{P}$ & 2.13 & 0.485 & 0.90 & 41 & & & & & $\begin{array}{c}{[9 \mathrm{c}, 12 \mathrm{i}, 12 \mathrm{w}, 22 \mathrm{~b}, 41 \mathrm{~b}, 41 \mathrm{~d}, 41 \mathrm{~g},} \\
50 \mathrm{i}, 50 \mathrm{~m}, 50 \mathrm{n}, 60]\end{array}$ \\
\hline
\end{tabular}




\begin{tabular}{|c|c|c|c|c|c|c|c|c|c|c|}
\hline 58 & Mo-P & 2.353 & 0.29 & 0.93 & 52 & & & & & $\begin{array}{c}{[12 \mathrm{v}-\mathrm{z}, 15 \mathrm{a}, 44 \mathrm{~b}, 44 \mathrm{~g}, 54 \mathrm{c}, 54 \mathrm{j}} \\
61]\end{array}$ \\
\hline 59 & $\mathrm{Ni}-\mathrm{S}$ & & & & & 1.915 & 0.55 & 0.91 & 43 & {$[25 \mathrm{i}, 281,52 \mathrm{~d}, 62]$} \\
\hline 60 & $\mathrm{Cu}-\mathrm{S}$ & 2.02 & 0.38 & 0.92 & 47 & 1.915 & 0.53 & 0.99 & 106 & [18h, 32ah, 40f, 58a, 63] \\
\hline 61 & V-V & 2.48 & 0.395 & 0.93 & 61 & & & & & {$[49 \mathrm{~d}, 64]$} \\
\hline 62 & $\mathrm{Cr}-\mathrm{Cr}$ & 2.38 & 0.465 & 0.95 & 126 & 1.97 & 0.49 & 0.91 & 38 & {$[40 \mathrm{c}, 48 \mathrm{~g}, 49 \mathrm{~d}, 64 \mathrm{e}-\mathrm{g}, 65]$} \\
\hline 63 & $\begin{array}{l}\mathrm{Mn}- \\
\mathrm{Mn}\end{array}$ & 2.17 & 0.54 & 0.93 & 79 & & & & & $\begin{array}{c}{[9 \mathrm{j}, 12 \mathrm{i}, 12 \mathrm{q}, 12 \mathrm{u}, 49 \mathrm{a}, 49 \mathrm{~b}, 49 \mathrm{~d},} \\
64 \mathrm{e}, 64 \mathrm{f}, 65 \mathrm{i}, 65 \mathrm{w}, 65 \mathrm{ac}, 66]\end{array}$ \\
\hline 64 & $\mathrm{Fe}-\mathrm{Fe}$ & & & & & 1.88 & 0.60 & 0.97 & 41 & $\begin{array}{c}{[12 \mathrm{~h}, 12 \mathrm{i}, 13 \mathrm{~d}, 49 \mathrm{~b}, 49 \mathrm{~d}, 50 \mathrm{j}, 64 \mathrm{e}} \\
\mathrm{g}, 65 \mathrm{a}, 65 \mathrm{i}, 66 \mathrm{f}, 67]\end{array}$ \\
\hline 65 & $\mathrm{Cu}-\mathrm{Cu}$ & & & & & 1.78 & 0.525 & 0.96 & 116 & $\begin{array}{c}{[32 \mathrm{f}, 32 \mathrm{k}, 32 \mathrm{z}, 49 \mathrm{~d}, 53 \mathrm{e}, 58 \mathrm{~b}-\mathrm{d},} \\
59 \mathrm{c}, 63 \mathrm{f}, 68]\end{array}$ \\
\hline 66 & $\mathrm{Nb}-\mathrm{Nb}$ & 2.65 & 0.408 & 0.88 & 41 & & & & & [49d, 64g, 64h, 67c, 69] \\
\hline 67 & $\begin{array}{l}\text { Mo- } \\
\text { Mo }\end{array}$ & 2.58 & 0.38 & 0.91 & 168 & 2.233 & 0.575 & 0.99 & 72 & $\begin{array}{c}{[12 \mathrm{v}, 12 \mathrm{y}, 34 \mathrm{c}, 34 \mathrm{e}, 34 \mathrm{f}, 34 \mathrm{j}, 34 \mathrm{l},} \\
34 \mathrm{o}, 48 \mathrm{~g}, 49 \mathrm{~d}, 54 \mathrm{c}, 54 \mathrm{n}, 54 \mathrm{o}, 61, \\
64 \mathrm{~g}, 65 \mathrm{~m}, 65 \mathrm{n}, 65 \mathrm{p}, 65 \mathrm{v}, 65 \mathrm{z}, \\
67 \mathrm{c}, 69 \mathrm{~b}, 70]\end{array}$ \\
\hline 68 & $\mathrm{Ru}-\mathrm{Ru}$ & & & & & 2.085 & 0.58 & 0.96 & 43 & $\begin{array}{c}34 \mathrm{c}, 49 \mathrm{~d}, 55 \mathrm{a}, 55 \mathrm{c}, 55 \mathrm{~h}, 65 \mathrm{~b}, \\
67 \mathrm{c}, 69 \mathrm{~b}, 71]\end{array}$ \\
\hline 69 & $\mathrm{~W}-\mathrm{W}$ & 2.628 & 0.375 & 0.96 & 81 & 2.27 & 0.65 & 0.99 & 18 & $\begin{array}{c}\text { [12ag, 36c, 48g, 56e, 56h, 64g, } \\
65 \mathrm{n}, 65 \mathrm{v}, 65 \mathrm{z}, 70 \mathrm{c}, 70 \mathrm{e}, 70 \mathrm{k}, 701, \\
70 \mathrm{v}, 72]\end{array}$ \\
\hline 70 & Re-Re & 2.505 & 0.27 & 0.90 & 100 & & & & & [48g, 65n, 65r, 70s, 70v, 73] \\
\hline 71 & $\mathrm{Au}-\mathrm{Au}$ & 2.41 & 0.41 & 0.91 & 49 & 2.225 & 0.49 & 0.97 & 79 & $\begin{array}{c}\text { [42k, } 53 \mathrm{n}, 58 \mathrm{~b}, 58 \mathrm{~d}, 59 \mathrm{c}, 59 \mathrm{~d}, \\
68 \mathrm{~h}, 74]\end{array}$ \\
\hline 72 & U-U & 3.255 & 0.658 & 0.86 & 95 & 2.46 & 0.44 & 0.96 & 25 & {$[37 \mathrm{e}, 37 \mathrm{~g}, 46 \mathrm{r}, 57 \mathrm{~d}, 65 \mathrm{z}, 70 \mathrm{k}, 75]$} \\
\hline
\end{tabular}


Table S2. The results of the $B O$ calculations for the $\mathrm{Re}_{6}$-cluster complexes.

\begin{tabular}{|c|c|c|c|c|c|c|c|c|c|c|c|}
\hline \multirow{2}{*}{$\mathrm{N}$} & \multirow{2}{*}{ Compound } & \multirow{2}{*}{ VEC * } & \multicolumn{3}{|c|}{ Bond length, $\AA$} & \multicolumn{3}{|c|}{ Bond order, v.u. } & \multicolumn{2}{|c|}{$\begin{array}{c}\text { Total electron } \\
\text { count for Re, v.u. }\end{array}$} & \multirow{2}{*}{$\begin{array}{c}\text { Cryst. } \\
\text { Struct. } \\
\text { ref. }\end{array}$} \\
\hline & & & Re-Re & Re-L ${ }^{i}$ & Re-L ${ }^{a}$ & Re-Re & Re-L ${ }^{i}$ & $\mathrm{Re}-\mathrm{L}^{\mathrm{a}}$ & Expect & Calcul & \\
\hline 1 & {$\left[\mathrm{Bu}_{4} \mathrm{~N}\right]\left[\mathrm{Re}_{6} \mathrm{~S}_{8} \mathrm{Cl}_{4}(\mathrm{py})_{2}\right]$} & 23 & 2.596 & 2.401 & \begin{tabular}{l|}
$\mathrm{N}: 2.161$ \\
$\mathrm{Cl}: 2.400$
\end{tabular} & 0.68 & 0.92 & $\begin{array}{l}\text { N: } 0.58 \\
\text { Cl: } 0.60\end{array}$ & 7 & 7.00 & [76] \\
\hline 2 & {$\left[\mathrm{Ph}_{4} \mathrm{P}\right]_{3}\left[\operatorname{Re}_{6} \mathrm{~S}_{8}(\mathrm{CN})_{6}\right]$} & 23 & 2.606 & 2.411 & 2.150 & 0.66 & 0.89 & 0.65 & 7 & 6.87 & [77] \\
\hline 3 & $\mathrm{Cs}_{6} \operatorname{Re}_{6} \mathrm{~S}_{15}$ & 24 & 2.605 & 2.403 & 2.451 & 0.66 & 0.92 & 0.80 & 7 & 7.10 & [78] \\
\hline 4 & {$\left[\mathrm{Bu}_{4} \mathrm{~N}\right]_{4}\left[\mathrm{Re}_{6} \mathrm{~S}_{8} \mathrm{Br}_{6}\right]$} & 24 & 2.592 & 2.395 & 2.595 & 0.69 & 0.94 & 0.68 & 7 & 7.19 & [79] \\
\hline 5 & {$\left[\mathrm{Bu}_{4} \mathrm{~N}\right]_{4}\left[\mathrm{Re}_{6} \mathrm{~S}_{8} \mathrm{I}_{6}\right]$} & 24 & 2.598 & 2.403 & 2.787 & 0.68 & 0.92 & 0.62 & 7 & 7.00 & [79] \\
\hline 6 & {$\left[\operatorname{Re}_{6} \mathrm{~S}_{8}\left(\mathrm{PEt}_{3}\right)_{4}(\mathrm{Br})_{2}\right]$} & 24 & 2.599 & 2.404 & $\begin{array}{c}\text { P: } 2.466 \\
\text { Br: } 2.550\end{array}$ & 0.67 & 0.91 & $\begin{array}{l}\text { P: } 0.98 \\
\text { Br: } 0.76\end{array}$ & 7 & 7.26 & [80] \\
\hline 7 & {$\left[\mathrm{Re}_{6} \mathrm{~S}_{8}\left(\mathrm{PEt}_{3}\right)_{6}\right] \mathrm{Br}_{2} * \mathrm{CH}_{2} \mathrm{Cl}_{2}$} & 24 & 2.614 & 2.405 & 2.483 & 0.64 & 0.91 & 0.94 & 7 & 7.13 & [80] \\
\hline 8 & $\mathrm{Cs}_{3} \mathrm{Na}\left[\mathrm{Re}_{6} \mathrm{~S}_{8}(\mathrm{CN})_{6}\right]$ & 24 & 2.602 & 2.411 & 2.119 & 0.67 & 0.90 & 0.71 & 7 & 6.97 & [81] \\
\hline 9 & {$\left[\mathrm{Ph}_{4} \mathrm{P}\right]_{4}\left[\mathrm{Re}_{6} \mathrm{~S}_{8}(\mathrm{CN})_{6}\right] *\left(\mathrm{CH}_{3} \mathrm{CN}\right)_{2}$} & 24 & 2.608 & 2.404 & 2.166 & 0.65 & 0.91 & 0.62 & 7 & 6.88 & [82] \\
\hline 10 & {$\left[\mathrm{Bu}_{4} \mathrm{~N}\right]_{4}\left[\mathrm{Re}_{6} \mathrm{~S}_{8} \mathrm{Cl}_{6}\right]$} & 24 & 2.603 & 2.404 & 2.450 & 0.66 & 0.91 & 0.55 & 7 & 6.86 & [79] \\
\hline 11 & {$\left[\mathrm{Bu}_{4} \mathrm{~N}\right]_{2}\left[\mathrm{Re}_{6} \mathrm{~S}_{8} \mathrm{Cl}_{4}(\mathrm{cpy})_{2}\right]$} & 24 & 2.593 & 2.400 & $\begin{array}{l}\mathrm{N}: 2.212 \\
\mathrm{Cl}: 2.423\end{array}$ & 0.69 & 0.92 & $\begin{array}{l}\mathrm{N}: 0.53 \\
\mathrm{Cl}: 0.59\end{array}$ & 7 & 7.03 & [76] \\
\hline 12 & {$\left[\mathrm{Bu}_{4} \mathrm{~N}\right]\left[\mathrm{Re}_{6} \mathrm{~S}_{8} \mathrm{Cl}_{3}(\mathrm{py})_{3}\right]$} & 24 & 2.594 & 2.401 & $\begin{array}{l}\mathrm{N}: 2.187 \\
\mathrm{Cl}: 2.424\end{array}$ & 0.69 & 0.92 & $\begin{array}{l}\mathrm{N}: 0.57 \\
\text { Cl: } 0.59\end{array}$ & 7 & 7.02 & [76] \\
\hline 13 & {$\left[\mathrm{Bu}_{4} \mathrm{~N}\right]_{2}\left[\mathrm{Re}_{6} \mathrm{~S}_{8} \mathrm{Cl}_{4}(\mathrm{ppy})_{2}\right]$} & 24 & 2.598 & 2.410 & $\begin{array}{l}\mathrm{N}: 2.216 \\
\mathrm{Cl}: 2.435\end{array}$ & 0.68 & 0.90 & $\begin{array}{l}\mathrm{N}: 0.53 \\
\mathrm{Cl}: 0.57\end{array}$ & 7 & 6.86 & [83] \\
\hline 14 & $\mathrm{Cs}_{6} \mathrm{Re}_{6} \mathrm{Se}_{15}$ & 24 & $\begin{array}{c}2.63 \\
4\end{array}$ & $\begin{array}{c}2.52 \\
3\end{array}$ & 2.574 & 0.59 & 0.94 & 0.82 & 7 & 6.95 & [84] \\
\hline 15 & $\mathrm{Re}_{6} \mathrm{Se}_{8}\left(\mathrm{PEt}_{3}\right)_{4}(\mathrm{I})_{2} * 2 \mathrm{CHCl}_{3}$ & 24 & 2.641 & 2.521 & $\begin{array}{l}\text { P: } 2.481 \\
\text { I: } 2.747\end{array}$ & 0.58 & 0.94 & $\begin{array}{l}\text { P: } 0.95 \\
\text { I: } 0.69\end{array}$ & 7 & 6.94 & [85] \\
\hline 16 & $\mathrm{Re}_{6} \mathrm{Se}_{8}\left(\mathrm{PEt}_{3}\right)_{4}(\mathrm{CN})_{2}$ & 24 & 2.647 & 2.522 & $\begin{array}{l}\text { P: } 2.478 \\
\text { C: } 2.104\end{array}$ & 0.56 & 0.94 & $\begin{array}{l}\text { P: } 0.95 \\
\text { C: } 0.75\end{array}$ & 7 & 6.91 & [86] \\
\hline 17 & {$\left[\operatorname{Re}_{6} \mathrm{Se}_{8}\left(\mathrm{PEt}_{3}\right)_{4}(\mathrm{CO})_{2}\right]\left[\mathrm{SbF}_{6}\right]_{2}$} & 24 & 2.640 & 2.518 & $\begin{array}{l}\text { P: } 2.484 \\
\text { C: } 2.060\end{array}$ & 0.58 & 0.95 & $\begin{array}{l}\text { P: } 0.94 \\
\text { C: } 0.85\end{array}$ & 7 & 7.04 & [87] \\
\hline 18 & $\mathrm{Re}_{6} \mathrm{Se}_{8}\left(\mathrm{PEt}_{3}\right)_{5}(\mathrm{CN})(\mathrm{BPh} 4)$ & 24 & 2.634 & 2.509 & $\begin{array}{l}\text { P: } 2.478 \\
\text { C: } 2.143\end{array}$ & 0.59 & 0.98 & $\begin{array}{l}\text { P: } 0.95 \\
\text { C: } 0.67\end{array}$ & 7 & 7.18 & [86] \\
\hline 19 & $\mathrm{Re}_{6} \mathrm{Te}_{15}$ & 24 & $\begin{array}{c}2.67 \\
6\end{array}$ & 2.692 & 2.710 & 0.51 & 1.02 & 0.98 & 7 & 7.09 & [88] \\
\hline 20 & {$[\mathrm{Mn}(\text { salen })]_{4 \mathrm{n}}\left[\operatorname{Re}_{6} \mathrm{Te}_{8}(\mathrm{CN})_{6}\right]_{\mathrm{n}}$} & 24 & $\begin{array}{c}2.69 \\
3\end{array}$ & $\begin{array}{c}2.69 \\
4\end{array}$ & 2.105 & 0.47 & 1.02 & C: 0.75 & 7 & 6.71 & [89] \\
\hline
\end{tabular}

$* \mathrm{VEC}=$ the total number of valence electrons of the cluster. 
Table S3. Bond valence parameters used in this work.

\begin{tabular}{|c|c|c|}
\hline \multirow{2}{*}{$i$-j pair } & \multicolumn{2}{|c|}{$\begin{array}{c}\text { Bond valence } \\
\text { parameter, } \AA\end{array}$} \\
\cline { 2 - 3 } & $R_{0 i j}$ & $b_{i j}$ \\
\hline Re-Re & 2.495 & 0.265 \\
\hline Re-N & 1.98 & 0.37 \\
\hline Re-C & 2.007 & 0.335 \\
\hline Re-Cl & 2.23 & 0.37 \\
\hline Re-S & 2.37 & 0.37 \\
\hline Re-Br & 2.45 & 0.37 \\
\hline Re-P & 2.46 & 0.37 \\
\hline Re-Se & 2.50 & 0.37 \\
\hline Re-I & 2.61 & 0.37 \\
\hline
\end{tabular}




\section{Electron descriptors calculated by Eqs 1 and 3 (Examples)}

12.1. Electron density at the bond critical points in 4-[[4-(methoxy)-3quinolinyl]thio]-3-thiomethylquinoline (See the fragment of the crystal structure).

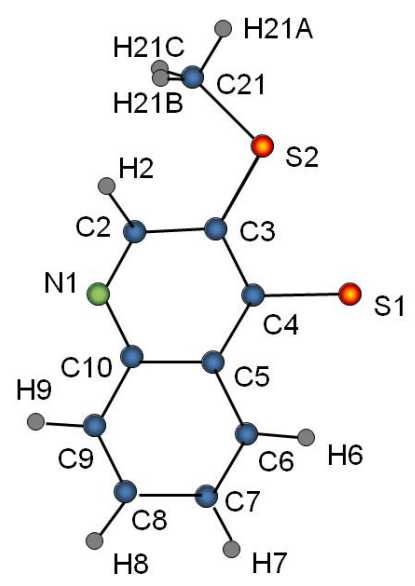

\begin{tabular}{|c|c|c|c|c|c|c|}
\hline \multirow[b]{2}{*}{ Bond } & \multirow{2}{*}{$\begin{array}{c}R_{i j}, \\
\AA\end{array}$} & \multirow{2}{*}{$\begin{array}{c}\rho_{c}, \mathrm{e} / \AA^{3} \\
\text { Eq. } 3 \\
\text { This } \\
\text { work }\end{array}$} & \multicolumn{2}{|c|}{$\begin{array}{l}\rho_{c}, \mathrm{e} / \AA^{3} \\
\text { Ref. }^{[90]}\end{array}$} & \multicolumn{2}{|c|}{$\begin{array}{c}\text { Relative } \\
\text { difference, \% }\end{array}$} \\
\hline & & & $\begin{array}{c}\text { Model } \\
\mathrm{I}^{*}\end{array}$ & $\begin{array}{l}\text { Model } \\
\text { II** }\end{array}$ & $\begin{array}{l}\text { This } \\
\text { work - } \\
\text { Model } \\
\text { I }\end{array}$ & $\begin{array}{c}\text { Model } \\
\text { I- } \\
\text { Model } \\
\text { II }\end{array}$ \\
\hline $\mathrm{S} 1-\mathrm{C} 4$ & 1.782 & 1.25 & 1.28 & 1.01 & 2.3 & 21.1 \\
\hline $\mathrm{S} 1-\mathrm{C} 13$ & 1.770 & 1.28 & 1.27 & 1.03 & 0.8 & 18.9 \\
\hline $\mathrm{S} 2-\mathrm{C} 3$ & 1.758 & 1.31 & 1.30 & 1.05 & 0.8 & 19.2 \\
\hline S2-C21 & 1.808 & 1.20 & 1.26 & 0.98 & 4.8 & 22.2 \\
\hline $\mathrm{C} 2-\mathrm{C} 3$ & 1.428 & 1.98 & 2.00 & 1.41 & 1.0 & 29.5 \\
\hline C3-C4 & 1.390 & 2.14 & 2.12 & 1.50 & 0.9 & 29.2 \\
\hline $\mathrm{C} 4-\mathrm{C} 5$ & 1.426 & 1.99 & 1.96 & 1.41 & 1.5 & 28.1 \\
\hline C5-C6 & 1.421 & 2.01 & 2.00 & 1.43 & 0.5 & 28.5 \\
\hline C6-C7 & 1.376 & 2.20 & 2.23 & 1.53 & 1.3 & 31.4 \\
\hline C7-C8 & 1.416 & 2.03 & 2.07 & 1.44 & 1.9 & 30.4 \\
\hline C8-C9 & 1.378 & 2.20 & 2.18 & 1.53 & 0.9 & 29.8 \\
\hline C9-C10 & 1.417 & 2.03 & 2.06 & 1.43 & 1.5 & 30.6 \\
\hline $\mathrm{N} 1-\mathrm{C} 2$ & 1.317 & 2.38 & 2.53 & 1.86 & 5.9 & 26.5 \\
\hline N1-C10 & 1.367 & 2.14 & 2.19 & 1.71 & 2.3 & 21.9 \\
\hline N11-C12 & 1.317 & 2.38 & 2.52 & 1.85 & 5.6 & 26.6 \\
\hline N11-C20 & 1.374 & 2.11 & 2.13 & 1.69 & 0.9 & 20.7 \\
\hline C21-H21A & 1.068 & 1.93 & 1.73 & 1.29 & 11.6 & 25.4 \\
\hline C21-H21B & 0.986 & 2.34 & 1.84 & 1.45 & 27.2 & 21.2 \\
\hline C21-H21C & 0.995 & 2.29 & 1.85 & 1.44 & 23.8 & 22.2 \\
\hline $\mathrm{C} 2-\mathrm{H} 2$ & 1.081 & 1.88 & 1.87 & 1.24 & 0.5 & 33.7 \\
\hline C6-H6 & 1.072 & 1.92 & 1.85 & 1.26 & 3.8 & 31.9 \\
\hline C7-H7 & 1.080 & 1.88 & 1.76 & 1.24 & 6.8 & 29.5 \\
\hline C8-H8 & 1.079 & 1.89 & 1.72 & 1.24 & 9.9 & 27.9 \\
\hline C9-H9 & 1.083 & 1.87 & 1.75 & 1.24 & 6.9 & 29.1 \\
\hline O1...H22B & 2.020 & 0.13 & & & & 0 \\
\hline H6...H16 & 2.201 & 0.08 & $\begin{array}{l}0.04 \\
0.08\end{array}$ & $\begin{array}{l}0.04 \\
0.03\end{array}$ & $\begin{array}{c}225.0 \\
0\end{array}$ & 62.5 \\
\hline $\mathrm{O} 1 \ldots \mathrm{O} 1$ & 3.178 & 0.06 & 0.04 & 0.03 & 50.0 & 25.0 \\
\hline
\end{tabular}


12.2. Electron density at the bond critical points in $5,8,9,10-T e t r a h y d r o-6 H-1,3-$ dioxolo[4,5-h]pyrrolo[2,1-b]-[3]benzazepine-11-carbonitrile



\begin{tabular}{|l|c|c|c|c|}
\hline \multicolumn{1}{|c|}{ Bond } & $\begin{array}{c}R_{i j}, \\
\AA\end{array}$ & $\begin{array}{c}\rho_{c^{c}} \text { e/ } \AA^{3} \\
\text { Eq. 3 } \\
\text { This work }\end{array}$ & $\begin{array}{c}\rho_{c} \text { e/ } \AA^{3} \\
\text { Ref. }{ }^{391]}\end{array}$ & $\begin{array}{c}\text { Relative } \\
\text { difference, } \\
\%\end{array}$ \\
\hline C19-O20 & 1.427 & 1.73 & 1.75 & 1.1 \\
C19-O18 & 1.435 & 1.70 & 1.65 & 3.0 \\
C16-O20 & 1.373 & 1.95 & 1.92 & 1.6 \\
C15-O18 & 1.378 & 1.93 & 1.90 & 1.6 \\
\hline C15-C16 & 1.385 & 2.17 & 2.19 & 0.9 \\
C17-C16 & 1.376 & 2.21 & 2.18 & $1 . .4$ \\
C14-C15 & 1.375 & 2.21 & 2.15 & 2.8 \\
C14-C13 & 1.422 & 2.01 & 1.99 & 1.0 \\
C17-C12 & 1.411 & 2.05 & 1.96 & 4.6 \\
C13-C12 & 1.411 & 2.05 & 1.97 & 4.1 \\
C12-C11 & 1.503 & 1.70 & 1.70 & 0 \\
C4-C13 & 1.480 & 1.78 & 1.66 & 7.2 \\
C4-C3 & 1.424 & 2.00 & 1.82 & 9.9 \\
C4-C5 & 1.389 & 2.15 & 2.05 & 4.9 \\
C10-C11 & 1.534 & 1.59 & 1.58 & 0.6 \\
C6-C5 & 1.513 & 1.66 & 1.59 & 4.4 \\
C6-C7 & 1.538 & 1.58 & 1.57 & 0.6 \\
C8-C7 & 1.530 & 1.61 & 1.60 & 0.6 \\
\hline C3-N21 & 1.165 & 3.29 & 3.42 & 3.8 \\
C10-N9 & 1.455 & 1.78 & 1.73 & 2.9 \\
C8-N9 & 1.465 & 1.74 & 1.63 & 6.7 \\
C5-N9 & 1.342 & 2.26 & 2.29 & 1.3 \\
\hline
\end{tabular}


12.3. Electron density at the bond critical points in $\mathrm{MeCp}(\mathrm{CO}) 2 \mathrm{Mn}=\mathrm{C}(\mathrm{OEt}) \mathrm{Me}$.

The first and the second rows are related to the experimental and theoretical data, respectively, of the original work.

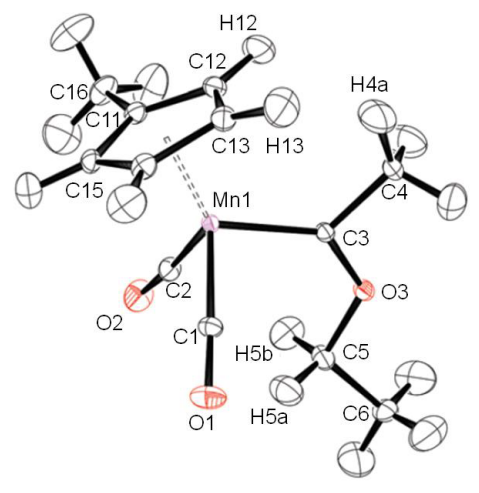

\begin{tabular}{|c|c|c|c|c|}
\hline Bond & $\begin{array}{c}R_{i j} \\
\AA\end{array}$ & $\begin{array}{c}\rho_{c}, \text { e } / \AA^{3} \\
\text { Eq. } 3 \\
\text { This } \\
\text { work }\end{array}$ & $\begin{array}{l}\rho_{c}, \text { e/ } \AA^{3} \\
\text { Ref. }^{[92]}\end{array}$ & $\begin{array}{c}\text { Relative } \\
\text { difference, } \\
\%\end{array}$ \\
\hline \multirow[t]{2}{*}{ Mn1-C1 } & 1.784 & 0.99 & 1.03 & 3.9 \\
\hline & 1.781 & 1.00 & 1.03 & 2.9 \\
\hline \multirow[t]{2}{*}{ Mn1-C2 } & 1.780 & 1.00 & 1.01 & 1.0 \\
\hline & 1.776 & 1.01 & 1.04 & 2.9 \\
\hline \multirow{2}{*}{ Mn1-C3 } & 1.901 & 0.78 & 0.99 & 21.2 \\
\hline & 1.900 & 0.78 & 0.89 & 12.4 \\
\hline \multirow[t]{2}{*}{ Mn1-C11 } & 2.167 & 0.45 & 0.41 & 9.8 \\
\hline & 2.169 & 0.45 & 0.50 & 10.0 \\
\hline Mn1-C12 & 2.153 & 0.46 & 0.50 & 8.0 \\
\hline \multirow[t]{2}{*}{ Mn1-C13 } & 2.160 & 0.46 & 0.41 & 12.2 \\
\hline & 2.151 & 0.47 & 0.49 & 4.1 \\
\hline \multirow[t]{2}{*}{ Mn1-C14 } & 2.160 & 0.46 & 0.40 & 15.0 \\
\hline & 2.145 & 0.47 & 0.52 & 9.6 \\
\hline Mn1-C15 & 2.157 & 0.46 & 0.49 & 6.1 \\
\hline \multirow[t]{2}{*}{$\mathrm{C} 1-\mathrm{O} 1$} & 1.161 & 3.09 & 3.47 & 11.0 \\
\hline & 1.158 & 3.11 & 3.09 & 0.6 \\
\hline \multirow[t]{2}{*}{$\mathrm{C} 2-\mathrm{O} 2$} & 1.164 & 3.07 & 3.49 & 12.0 \\
\hline & 1.160 & 3.10 & 3.07 & 1.0 \\
\hline \multirow[t]{2}{*}{$\mathrm{C} 3-\mathrm{C} 4$} & 1.516 & 1.43 & 1.69 & 15.4 \\
\hline & 1.508 & 1.45 & 1.78 & 18.5 \\
\hline \multirow[t]{2}{*}{$\mathrm{C} 3-\mathrm{O} 3$} & 1.334 & 2.12 & 2.15 & 1.4 \\
\hline & 1.331 & 2.14 & 2.01 & 6.5 \\
\hline \multirow[t]{2}{*}{$\mathrm{C} 5-\mathrm{O} 3$} & 1.442 & 1.68 & 1.64 & 2.4 \\
\hline & 1.436 & 1.70 & 1.63 & 4.3 \\
\hline \multirow[t]{2}{*}{ C5-C6 } & 1.508 & 1.68 & 1.77 & 5.1 \\
\hline & 1.502 & 1.70 & 1.78 & 4.5 \\
\hline \multirow[t]{2}{*}{ C11-C12 } & 1.424 & 2.00 & 2.09 & 4.3 \\
\hline & 1.417 & 2.03 & 2.01 & 1.0 \\
\hline \multirow[t]{2}{*}{ C11-C15 } & 1.430 & 1.97 & 2.07 & 4.8 \\
\hline & 1.425 & 2.00 & 1.98 & 1.0 \\
\hline \multirow{2}{*}{ C11-C16 } & 1.498 & 1.72 & 1.75 & 1.7 \\
\hline & 1.487 & 1.76 & 1.80 & 2.2 \\
\hline \multirow[t]{2}{*}{$\mathrm{C} 12-\mathrm{C} 13$} & 1.427 & 1.99 & 2.01 & 1.0 \\
\hline & 1.418 & 2.02 & 1.99 & 1.5 \\
\hline \multirow{2}{*}{ C13-C14 } & 1.422 & 2.01 & 2.00 & 0.5 \\
\hline & 1.417 & 2.03 & 2.00 & 1.5 \\
\hline \multirow{2}{*}{ C14-C15 } & 1.420 & 2.02 & 2.04 & 1.0 \\
\hline & 1.412 & 2.05 & 2.02 & 1.5 \\
\hline
\end{tabular}




\begin{tabular}{|c|c|c|c|c|}
\hline \multirow[t]{2}{*}{$\mathrm{C} 12-\mathrm{H} 12 \mathrm{a}$} & 1.083 & 1.87 & 1.82 & 2.7 \\
\hline & 1.078 & 1.89 & 1.95 & 3.1 \\
\hline \multirow{2}{*}{ C13-H13a } & 1.083 & 1.87 & 1.86 & 0.5 \\
\hline & 1.077 & 1.89 & 1.95 & 3.1 \\
\hline \multirow[t]{2}{*}{ C14-H14a } & 1.083 & 1.87 & 1.85 & 1.1 \\
\hline & 1.077 & 1.89 & 1.96 & 3.6 \\
\hline \multirow[t]{2}{*}{ C15-H15a } & 1.083 & 1.87 & 1.87 & 0 \\
\hline & 1.078 & 1.89 & 1.95 & 3.1 \\
\hline \multirow[t]{2}{*}{ C16-H16a } & 1.093 & 1.83 & 1.78 & 2.8 \\
\hline & 1.087 & 1.85 & 1.90 & 2.6 \\
\hline \multirow[t]{2}{*}{ C16-H16b } & 1.093 & 1.83 & 1.76 & 4.0 \\
\hline & 1.091 & 1.83 & 1.88 & 2.7 \\
\hline \multirow[t]{2}{*}{ C16-H16c } & 1.093 & 1.83 & 1.74 & 5.2 \\
\hline & 1.088 & 1.85 & 1.91 & 3.1 \\
\hline \multirow[t]{2}{*}{ C1-H5a } & 2.410 & 0.09 & 0.08 & 12.5 \\
\hline & 2.422 & 0.09 & 0.08 & 12.5 \\
\hline \multirow[t]{2}{*}{$\mathrm{C} 2-\mathrm{H} 5 \mathrm{~b}$} & 2.530 & 0.07 & 0.09 & 22.2 \\
\hline & 2.515 & 0.07 & 0.08 & 12.5 \\
\hline O1-H4a_4 & 2.520 & 0.03 & 0.02 & 50.0 \\
\hline $\mathrm{O} 2-\mathrm{H} 5 \mathrm{~b} \_3$ & 2.600 & 0.03 & 0.03 & 0 \\
\hline $\mathrm{O} 2-\mathrm{H} 5 \mathrm{a}^{-} 3$ & 2.515 & 0.03 & 0.02 & 50.0 \\
\hline $\mathrm{H} 4 \mathrm{a}-\mathrm{O} 1{ }^{-} 4$ & 2.520 & 0.03 & 0.02 & 50.0 \\
\hline $\mathrm{H} 5 \mathrm{~b}-\mathrm{O} 2-3$ & 2.600 & 0.03 & 0.03 & 0 \\
\hline $\mathrm{H} 15 \mathrm{a}-\mathrm{O} \overline{2} \_1$ & 2.515 & 0.03 & 0.02 & 50.0 \\
\hline $\mathrm{H} 4 \mathrm{a}-\mathrm{H} 12 \mathrm{a}$ & 2.35 & 0.05 & 0.05 & 0 \\
\hline H4a-H13a & 2.14 & 0.07 & 0.04 & 75.0 \\
\hline H4c-H6a_4 & 2.24 & 0.06 & 0.02 & 200.0 \\
\hline H5a-H12a_4 & 2.558 & 0.03 & 0.03 & 0 \\
\hline H6a-H4c_4 & 2.24 & 0.06 & 0.01 & 500.0 \\
\hline H6b-H15a_1 & 2.347 & 0.05 & 0.03 & 66.7 \\
\hline H6c-H13a_3 & 2.344 & 0.05 & 0.01 & 400.0 \\
\hline H16a-H16b_2 & 2.478 & 0.03 & 0.02 & 50.0 \\
\hline H16b-H16a_2 & 2.478 & 0.03 & 0.02 & 50.0 \\
\hline H13a-H6c_3 & 2.344 & 0.05 & 0.01 & 400.0 \\
\hline H15a-H6b_1 & 2.347 & 0.05 & 0.03 & 66.7 \\
\hline
\end{tabular}


12.4. Bond orders in the $\mathrm{W}_{2}{ }^{6+}$ paddlewheel complex.



\begin{tabular}{|l|c|c|c|c|}
\hline Bond & $\begin{array}{c}R_{i j}, \\
\AA\end{array}$ & $\begin{array}{c}B O, \text { v.u. } \\
\text { Eq. 1 } \\
\text { This } \\
\text { work }\end{array}$ & $\begin{array}{c}\text { Wiberg } B O, \\
\text { v.u. } \\
\text { Ref. }^{[12 a g]}\end{array}$ & $\begin{array}{c}\text { Relative } \\
\text { difference, } \\
\%\end{array}$ \\
\hline W-W & 2.175 & 3.35 & 3.31 & 1.2 \\
W-N & 2.131 & 0.48 & 0.53 & 9.4 \\
\hline
\end{tabular}

12.5. Bond orders in $\mathrm{Mn}_{2} \mathrm{C}_{8} \mathrm{Me}_{6}$.

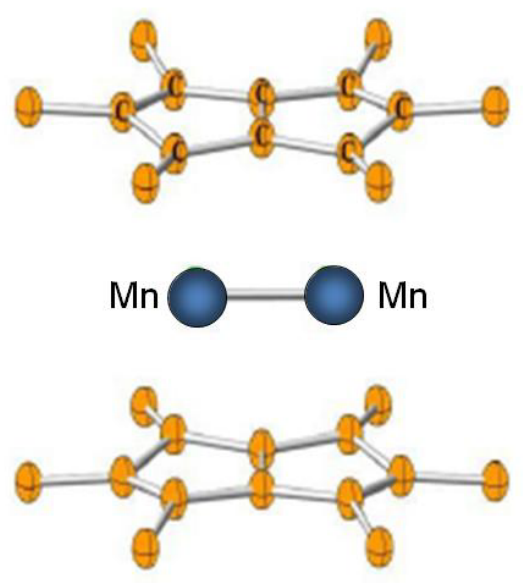

\begin{tabular}{|c|c|c|c|c|c|c|}
\hline & & \multirow{2}{*}{$\begin{array}{c}\text { BO, v.u. } \\
\text { Eq. } 1\end{array}$} & \multicolumn{2}{|c|}{$\begin{array}{c}\text { BO, v.u. } \\
\text { Ref. }\end{array}$} & \multicolumn{2}{|c|}{$\begin{array}{c}\text { Relative } \\
\text { Bifference, \% }\end{array}$} \\
\cline { 5 - 8 } & \multirow{2}{*}{$R_{i j}, \AA \begin{array}{c}\text { This } \\
\text { work }\end{array}$} & DI & Wiberg & $\begin{array}{c}\text { This } \\
\text { work- } \\
\text { Wiberg }\end{array}$ & $\begin{array}{c}\text { Wiberg- } \\
\text { DI }\end{array}$ \\
& & & & & & \\
\hline Mn1-Mn2 & 2.202 & 0.94 & 1.11 & 1.23 & 23.6 & 9.8 \\
Mn1-C1 & 2.053 & 0.47 & 0.50 & 0.64 & 26.6 & 21.9 \\
Mn1-C2 & 2.060 & 0.46 & 0.42 & 0.58 & 20.7 & 27.6 \\
Mn1-C3 & 2.053 & 0.47 & 0.50 & 0.64 & 26.6 & 21.9 \\
Mn1-C4 & 2.161 & 0.33 & 0.29 & 0.40 & 12.1 & 21.2 \\
Mn1-C5 & 2.161 & 0.33 & 0.29 & 0.40 & 12.1 & 21.2 \\
\hline
\end{tabular}




\subsection{Bond orders in the planar uranium oxides.}

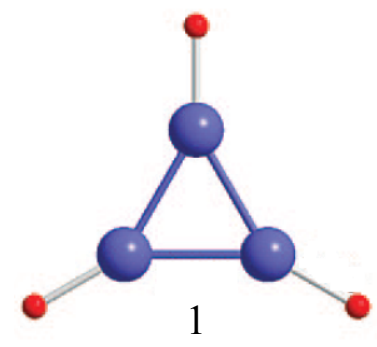

1



Balance of the valence electrons:

The sum of valence electrons for $U$ atom should be equal to 8 :

6 (uranium electrons) +2 (oxygen electrons) Each $U$ is bonded to oxygen atom and two other $U$ atoms. The sum of bond orders for the three bonds should be equal to 8 v.u.

\begin{tabular}{|c|c|c|c|c|c|c|c|}
\hline $\mathrm{N}$ & Compound & Bond & $R_{i j}, \AA$ & $\begin{array}{c}B O, \text { v.u. } \\
\text { Eq. } 1 \\
\text { This work }\end{array}$ & $\begin{array}{l}\text { Discrepancy } \\
\text { in the number } \\
\text { of valence } \\
\text { electrons, v.u. }\end{array}$ & $\begin{array}{l}\text { Wiberg } \\
\text { Bond } \\
\text { Index, v.u. } \\
\text { Ref. }{ }^{[93]}\end{array}$ & $\begin{array}{l}\text { Discrepancy in } \\
\text { the number of } \\
\text { valence } \\
\text { electrons, v.u. }\end{array}$ \\
\hline \multirow[t]{2}{*}{1} & \multirow{2}{*}{ cyclo- $\mathrm{U}_{3} \mathrm{O}_{3}$} & $\begin{array}{l}\text { U-U } \\
\text { U-O }\end{array}$ & $\begin{array}{l}2.539 \\
1.833\end{array}$ & $\begin{array}{l}2.898 \\
1.888\end{array}$ & & $\begin{array}{l}2.735 \\
2.028\end{array}$ & \\
\hline & & $B V S$ & & 7.684 & 0.316 & 7.498 & 0.502 \\
\hline \multirow[t]{2}{*}{2} & \multirow[t]{2}{*}{ cyclo- $\mathrm{U}_{4} \mathrm{O}_{4}$} & $\begin{array}{l}\text { U-U } \\
\text { U-O }\end{array}$ & $\begin{array}{l}2.539 \\
1.830\end{array}$ & $\begin{array}{l}2.898 \\
1.902\end{array}$ & & $\begin{array}{l}2.478 \\
2.059\end{array}$ & \\
\hline & & $B V S$ & & 7.698 & 0.302 & 7.015 & 0.985 \\
\hline
\end{tabular}



aE. Espinosa, E. Molins, C. Lecomte, Chem. Phys. Lett. 1998, 285, 170-173; bE. Espinosa, I. Alkorta, J. Elguero, E. Molins, J. Chem. Phys. 2002, 117, 5529-5542. R. F. W. Bader, Atoms in Molecules: A Quantum Theory, Clarendon Press, 1994. F. D. Hardcastle, I. E. Wachs, J. Raman Spectrosc. 1990, 21, 683-691.

aC. V. J. Skipper, T. K. A. Hoang, D. M. Antonelli, N. Kaltsoyannis, Chem. Eur. J. 2012, 18, 17501760; bM. C. F. Wander, B. R. Bickmore, L. Lind, C. Andros, J. Hunt, H. Checketts, T. Goodell, Am. Mineral. 2015, 100, 160-171; cD. J. Wolstenholme, C. F. Matta, T. S. Cameron, J. Phys. Chem. A 2007, 111, 8803-8813; dJ. Oláh, F. Blockhuys, T. Veszprémi, C. Van Alsenoy, Eur. J. Inorg. Chem. 2006, 2006, 69-77; eP. L. A. Popelier, J. Phys. Chem. A 1998, 102, 1873-1878; fE. A. Zhurova, C. F. Matta, N. Wu, V. V. Zhurov, A. A. Pinkerton, J. Am. Chem. Soc. 2006, 128, 8849-8861; gA. S. Goloveshkin, I. S. Bushmarinov, A. A. Korlyukov, M. I. Buzin, V. I. Zaikovskii, N. D. Lenenko, A. S. Golub, Langmuir 2015, 31, 8953-8960; hC. V. J. Skipper, D. M. Antonelli, N. Kaltsoyannis, J. Phys. Chem. C 2012, 116, 19134-19144; iI. E. Golub, O. A. Filippov, E. I. Gutsul, N. V. Belkova, L. M. Epstein, A. Rossin, M. Peruzzini, E. S. Shubina, Inorg. Chem. 2012, 51, 6486-6497; jD. M. Camaioni, B. Ginovska-Pangovska, G. K. Schenter, S. M. Kathmann, T. Autrey, J. Phys. Chem. A 2012, 116, 7228-7237; kP. R. Varadwaj, A. Varadwaj, G. H. Peslherbe, H. M. Marques, J. Phys. Chem. A 2011, 115, 13180-13190; IL. Rincón, R. Almeida, J. Phys. Chem. A 1998, 102, 9244-9254; mC. F. Matta, N. Castillo, R. J. Boyd, J. Phys. Chem. A 2005, 109, 3669-3681; nN. Mandal, S. M. Pratik, A. Datta, J. Phys. Chem. B 2017, 121, 825834; oP. Panini, D. Chopra, Cryst. Growth Des. 2014, 14, 3155-3168.

[6] aE. Espinosa, I. Alkorta, I. Mata, E. Molins, J. Phys. Chem. A 2005, 109, 6532-6539; bL. Zhang, F. Ying, W. Wu, P. C. Hiberty, S. Shaik, Chem. Eur. J. 2009, 15, 2979-2989; cS. J. Grabowski, Crystals 2015, 6, 3; dN. J. M. Amezaga, S. C. Pamies, N. M. Peruchena, G. L. Sosa, J. Phys. Chem. A 2010, 114, 552-562; eN. N. Chipanina, N. F. Lazareva, T. N. Aksamentova, E. P. Doronina, B. A. Shainyan, J. Phys. Org. Chem. 2014, 27, 892-901; fR. Bianchi, A. Forni, T. Pilati, Chem. Eur. J. 2003, 9, 1631-1638. aM. V. Vener, A. V. Manaev, A. N. Egorova, V. G. Tsirelson, J. Phys. Chem. A 2007, 111, 1155-1162; bR. N. Singh, P. Rawat, J. Mol. Struct. 2013, 1054-1055, 65-75; cM. M. Montero-Campillo, A. M. Lamsabhi, O. Mó, M. Yáñez, J. Mol. Model. 2013, 19, 2759-2766; dA. T. Dubis, P. Stasiewicz, K. Pogorzelec-Glaser, A. Lapiński, J. Phys. Org. Chem. 2015, 28, 652-662; eG. Villani, PCCP 2013, 15, 19242-19252; fP. Li, W. Niu, T. Gao, H. Wang, ChemPhysChem 2014, 15, 3078-3088; gS. B. Novaković, G. A. Bogdanović, B. Fraisse, N. E. Ghermani, N. Bouhmaida, A. Spasojević-de Biré, J. Phys. Chem. A 2007, 111, 13492-13505; hP. Munshi, T. N. Guru Row, Acta Cryst. B. 2006, 62, 612-626; iX. Xu, PhD thesis, Ecole Centrale Paris 2015; jM. C. Daza, J. Dobado, J. M. Molina, J. L. Villaveces, PCCP 2000, 2, 4089-4094; kL. Guillaumes, P. Salvador, S. Simon, J. Phys. Chem. A 2014, $118,1142-$ 1149; IM. Palusiak, S. Simon, M. Solà, J. Org. Chem. 2006, 71, 5241-5248; mJ. Poater, X. Fradera, M. Sola, M. Duran, S. Simon, Chem. Phys. Lett. 2003, 369, 248-255; nM. Palusiak, S. Simon, M. Sola, Chem. Phys. 2007, 342, 43-54; oH. Raissi, A. F. Jalbout, M. A. Nasseria, M. Yoosefian, H. Ghiassi, A. J. Hameed, Int. J. Quantum Chem 2008, 108, 1444-1451; pJ. L. Perez-Lustres, M. Bräuer, M. Mosquera, T. Clark, PCCP 2001, 3, 3569-3579; qI. Majerz, Mol. Phys. 2007, 105, 2305-2314; rH. Karabıyık, R. Sevinçek, H. Petek, M. Aygün, J. Mol. Model. 2011, 17, 1295-1309; sP. R. Mallinson, G. T. Smith, C. C. Wilson, E. Grech, K. Wozniak, J. Am. Chem. Soc. 2003, 125, 4259-4270; tA. Nash, J. Saßmannshausen, L. Bozec, H. L. Birch, N. H. de Leeuw, J. Biomol. Struct. Dyn. 2017, 35, 1127-1137; uG. Saleh, PhD thesis, Milano, Italy 2014; vP. Roversi, F. Merati, R. Destro, M. Barzaghi, Can. J. Chem. 1996, 74, 1145-1161; wL. Bertini, V. Barbieri, P. Fantucci, L. D. Gioia, G. Zampella, Dalton Trans. 2011, 40, 7704-7712; xS. J. Grabowski, W. A. Sokalski, J. Leszczynski, J. Phys. Chem. A 2006, 110, 4772-4779; yY. H. Mariam, R. N. Musin, J. Phys. Chem. A 2008, 112, 134-145; zM. Lukomska, A. J. Rybarczyk-Pirek, M. Jablonski, M. Palusiak, PCCP 2015, 17, 16375-16387; aaM. Vener, E. Levina, A. Astakhov, V. Tsirelson, Chem. Phys. Lett. 2015, 638, 233-236; abV. V. Zhurov, A. A. Pinkerton, Z. Anorg. Allg. Chem. 2013, 639, 1969-1978; acA. Nowroozi, E. Nakhaei, E. Masumian, Struct. Chem. 2014, 25, 1415-1422; adA. Nowroozi, H. Raissi, H. Hajiabadi, P. M. Jahani, Int. J. Quantum Chem 2011, 111, 3040-3047; aeK. Sutter, G. A. Aucar, J. Autschbach, Chem. Eur. J. 2015, 21, 18138-18155. aV. G. Tsirelson, E. V. Bartashevich, A. I. Stash, V. A. Potemkin, Acta Crystallographica Section B: Structural Science 2007, 63, 142-150; bD. Devarajan, PhD thesis, Philipps-Universität Marburg 2011; cJ. S. Al-Otaibi, SpringerPlus 2015, 4, 586; dM. Kubicki, T. Borowiak, G. Dutkiewicz, M. Souhassou, C. Jelsch, C. Lecomte, J. Phys. Chem. B 2002, 106, 3706-3714; eC. Merkens, F. Pan, U. Englert, CrystEngComm 2013, 15, 8153-8158; fV. Legrand, S. Pillet, M. Souhassou, N. Lugan, C. Lecomte, J. Am. Chem. Soc. 2006, 128, 13921-13931; gY.-R. Guo, Q. Wu, S. O. Odoh, G. Schreckenbach, Q.-J. Pan, Inorg. Chem. 2013, 52, 9143-9152; hP. V. Bharatam, P. Iqbal, J. Comput. Chem. 2006, 27, 334343; iG. Rajalakshmi, V. R. Hathwar, P. Kumaradhas, Acta Cryst. B. 2014, 70, 331-341; jJ. Dobado, H. Martínez-García, J. M. Molina, M. R. Sundberg, J. Am. Chem. Soc. 1998, 120, 8461-8471; kJ. M. V. Franco, PhD thesis, Friedrich-Wilhelms-Universität Bonn, Germany 2015; II. Mata, E. Molins, I. 
Alkorta, E. Espinosa, J. Phys. Chem. A 2007, 111, 6425-6433; mI. Rozas, PCCP 2007, 9, 2782-2790; nK. K. Pandey, New J. Chem. 2016, 40, 7831-7839; oM. D. Esrafili, F. Mohammadian-Sabet, Can. J. Chem. 2014, 92, 1151-1156; pN. N. Chipanina, N. F. Lazareva, T. N. Aksamentova, A. Y. Nikonov, B. A. Shainyan, Organometallics 2014, 33, 2641-2652; qM. Liu, Q. Li, S. Scheiner, PCCP 2017, 19, 55505559; rS. J. Grabowski, ChemPhysChem 2014, 15, 876-884; sY. V. Nelyubina, A. A. Korlyukov, K. A. Lyssenko, ChemPhysChem 2015, 16, 676-681; tI. B. Dorofeeva, O. G. Tarakanov, Russ. J. Struc. Chem. 1984, 25, 23-28; uV. Tognetti, L. Joubert, J. Phys. Chem. A 2011, 115, 5505-5515; vM. Szaleniec, A. Drzewiecka-Matuszek, M. Witko, P. Hejduk, J. Mol. Model. 2013, 19, 4487-4501.

aP. Macchi, A. Sironi, in The Quantum Theory of Atoms in Molecules, Wiley-VCH Verlag GmbH \& Co. KGaA, 2007, pp. 343-374; bX. Fradera, M. A. Austen, R. F. Bader, J. Phys. Chem. A 1999, 103, 304-314; cL. F. Veiros, M. J. Calhorda, Dalton Trans. 2011, 40, 11138-11146; dL. H. Dos Santos, A. Lanza, A. M. Barton, J. Brambleby, W. J. Blackmore, P. A. Goddard, F. Xiao, R. C. Williams, T. Lancaster, F. L. Pratt, J. Am. Chem. Soc. 2016, 138, 2280-2291; eC. G. Gianopoulos, B. Zarychta, S. Cenedese, V. V. Zhurov, A. A. Pinkerton, J. Phys. Chem. A 2016, 120, 4059-4070; fJ. Overgaard, H. F. Clausen, J. A. Platts, B. B. Iversen, J. Am. Chem. Soc. 2008, 130, 3834-3843; gW. Scherer, P. Sirsch, D. Shorokhov, M. Tafipolsky, G. S. McGrady, E. Gullo, Chem. Eur. J. 2003, 9, 6057-6070; hI. Vidal, S. Melchor, I. Alkorta, J. Elguero, M. R. Sundberg, J. A. Dobado, Organometallics 2006, 25, 5638-5647; iJ. Hey, D. Leusser, D. Kratzert, H. Fliegl, J. M. Dieterich, R. A. Mata, D. Stalke, PCCP 2013, 15, 20600-20610; jU. Flierler, M. Burzler, D. Leusser, J. Henn, H. Ott, H. Braunschweig, D. Stalke, Angew. Chem. Int. Ed. 2008, 47, 4321-4325; kJ. M. Molina, J. A. Dobado, S. Melchor, J. Mol. Struct. THEOCHEM 2002, 589, 337-347; IJ. M. Cole, PhD thesis, Durham University, England 1997; mY. A. Abramov, L. Brammer, W. T. Klooster, R. M. Bullock, Inorg. Chem. 1998, 37, 6317-6328; nI. Alkorta, I. Rozas, J. Elguero, Struct. Chem. 1998, 9, 243-247; oJ. A. Platts, S. T. Howard, J. Chem. Soc., Perkin Trans. 1997, 2241-2248; pJ. Poater, M. Solà, M. Duran, X. Fradera, J. Phys. Chem. A 2001, 105, 20522063; qD. S. Yufit, J. A. Howard, M. G. Davidson, J. Chem. Soc., Perkin Trans. 2 2000, 249-253; rT. Q. Teodoro, R. L. A. Haiduke, RSC Advances 2014, 4, 39853-39859; sN. Ramanathan, K. Sundararajan, K. Vidya, E. D. Jemmis, Spectrochim. Acta, Part A 2016, 157, 69-78; tM. Zhang, Z. Liang, J. Ling, X. Ni, Z. Shen, Dalton Trans. 2015, 44, 11182-11190.

[10] aG. S. McGrady, P. Sirsch, N. P. Chatterton, A. Ostermann, C. Gatti, S. Altmannshofer, V. Herz, G. Eickerling, W. Scherer, Inorg. Chem. 2009, 48, 1588-1598; bC. Hauf, J. E. Barquera-Lozada, P. Meixner, G. Eickerling, S. Altmannshofer, D. Stalke, T. Zell, D. Schmidt, U. Radius, W. Scherer, Z. Anorg. Allg. Chem. 2013, 639, 1996-2004; cI. Alkorta, I. Rozas, J. Elguero, Int. J. Quantum Chem 2002, 86, 122-129; dW. Scherer, G. Eickerling, M. Tafipolsky, G. S. McGrady, P. Sirsch, N. P. Chatterton, Chem. Commun. 2006, 2986-2988; eA. Jana, D. Leusser, I. Objartel, H. W. Roesky, D. Stalke, Dalton Trans. 2011, 40, 5458-5463; fS. Grabowsky, PhD thesis, Freie Universität Berlin 2010; gM. Dakkouri, V. Novikov, L. Vilkov, J. Mol. Struct. 2010, 978, 234-245; hK. K. Pandey, C. Jones, Organometallics 2013, 32, 3395-3403; iI. Corral, O. Mó, M. Yáñez, New J. Chem. 2003, 27, 1657-1664; jM. Lein, J. A. Harrison, A. J. Nielson, Dalton Trans. 2011, 40, 10731-10741; kW. Scherer, P. Meixner, J. E. Barquera-Lozada, C. Hauf, A. Obenhuber, A. Brück, D. J. Wolstenholme, K. Ruhland, D. Leusser, D. Stalke, Angew. Chem. Int. Ed. 2013, 52, 6092-6096; IS. K. Ignatov, N. H. Rees, S. R. Dubberley, A. G. Razuvaev, P. Mountford, G. I. Nikonov, Chem. Commun. 2004, 952-953; mX. Ning, J. Wang, H. Wei, J. Phys. Chem. A 2016, 120, 4167-4178; nT. Stahl, P. Hrobárik, C. D. F. Königs, Y. Ohki, K. Tatsumi, S. Kemper, M. Kaupp, H. F. Klare, M. Oestreich, Chem. Sci. 2015, 6, 4324-4334; oM. I. Arz, G. Schnakenburg, A. Meyer, O. Schiemann, A. C. Filippou, Chem. Sci. 2016, 7, 4973-4979; pK. Petrov, PhD thesis, Budapest University of Technology and Economics 2012; qM. Hagemann, A. Mix, R. J. Berger, T. Pape, N. W. Mitzel, Inorg. Chem. 2008, 47, 10554-10564; rF. P. Arnold, J. Organomet. Chem. 2001, 617, 647-655; sR. J. Gillespie, P. L. A. Popelier, Chemical Bonding and Molecular Geometry: From Lewis to Electron Densities, Oxford University Press, 2001; tT. Szilvási, PhD thesis, Budapest University of Technology and Economics 2015; uJ. Xu, Y.-H. Ding, Aust. J. Chem. 2014, 67, 740-748; vT. Fukuda, H. Hashimoto, S. Sakaki, H. Tobita, Angew. Chem. 2016, 128, 196-200; wI. Corral, O. Mó, M. Yáñez, Int. J. Mass spectrom. 2003, 227, 401-412; xV. Montiel-Palma, O. Piechaczyk, A. Picot, A. Auffrant, L. Vendier, P. Le Floch, S. Sabo-Etienne, Inorg. Chem. 2008, 47, 8601-8603; yS. F. Vyboishchikov, G. I. Nikonov, Organometallics 2007, 26, 4160-4169; zW. Scherer, P. Meixner, K. Batke, J. E. Barquera-Lozada, K. Ruhland, A. Fischer, G. Eickerling, K. Eichele, Angew. Chem. Int. Ed. 2016, 55, 11673-11677; aaM. Jabłoński, Comput. Theor. Chem. 2016, 1096, 54-65; abQ.Z. Li, H.-Y. Zhuo, H.-B. Li, Z.-B. Liu, W.-Z. Li, J.-B. Cheng, J. Phys. Chem. A 2014, 119, 2217-2224; acD. J. Wolstenholme, P. D. Prince, G. S. McGrady, M. J. Landry, J. W. Steed, Inorg. Chem. 2011, 50, 11222-11227; adM. D. Esrafili, F. Mohammadian-Sabet, J. Mol. Model. 2015, 21, 60; aeA. Y. Khalimon, Z. H. Lin, R. Simionescu, S. F. Vyboishchikov, G. I. Nikonov, Angew. Chem. Int. Ed. 2007, 46, 4530-4533; afY. Horbatenko, PhD thesis, Girona University 2013. 
[11] aB. Bankiewicz, M. Palusiak, Comput. Theor. Chem. 2011, 966, 113-119; bP. Gómez, O. Gálvez, R. Escribano, PCCP 2009, 11, 9710-9719; cM. Jabłoński, M. Palusiak, J. Phys. Chem. A 2012, 116, 23222332; dM. Domagała, P. Matczak, M. Palusiak, Comput. Theor. Chem. 2012, 998, 26-33; eR. Flaig, T. Koritsanszky, B. Dittrich, A. Wagner, P. Luger, J. Am. Chem. Soc. 2002, 124, 3407-3417; fJ. Saßmannshausen, Dalton Trans. 2014, 43, 11195-11201; gP. Lipkowski, S. J. Grabowski, J. Leszczynski, J. Phys. Chem. A 2006, 110, 10296-10302; hX. An, B. Jing, Q. Li, J. Phys. Chem. A 2010, 114, 6438-6443; iJ. R. Mora, J. Lezama, E. Márquez, L. Escalante, T. Córdova, G. Chuchani, J. Phys. Org. Chem. 2011, 24, 229-240; jW. Seifert, S. Schwetlick, J. Reinhold, E. Butter, J. Cryst. Growth 1984, 66, 333-337; kY. G. Wang, C. Matta, N. H. Werstiuk, J. Comput. Chem. 2003, 24, $1720-1729$; IB. Bankiewicz, P. Matczak, M. Palusiak, J. Phys. Chem. A 2012, 116, 452-459; mE. Matito, J. Poater, M. Solá, in The Quantum Theory of Atoms in Molecules, Wiley-VCH Verlag GmbH \& Co. KGaA, 2007, pp. 399-423; nM. Vatanparast, Comput. Theor. Chem. 2014, 1048, 77-83; oD. Dey, S. Bhandary, A. Sirohiwal, V. R. Hathwar, D. Chopra, Chem. Commun. 2016, 52, 7225-7228; pY. Xia, A. S. Dudnik, V. Gevorgyan, Y. Li, J. Am. Chem. Soc. 2008, 130, 6940-6941; qL. Koskinen, S. Jääskeläinen, P. Hirva, M. Haukka, Solid State Sciences 2014, 35, 81-87; rG. V. Baryshnikov, S. V. Bondarchuk, B. F. Minaev, L. V. Kovtun, A. M. Malachov, Bull. Cherkasy Univ., Chem. Sci. 2013, 11-19.

[12] aG. La Penna, S. Furlan, M. Solà, Theor. Chem. Acc. 2011, 130, 27; bC. L. Firme, J. Brazilian Chem. Soc. 2012, 23, 513-521; cT. Papp, L. Kollár, T. Kégl, J. Quantum Chem. 2014, 2014; dP. Macchi, A. Sironi, Coord. Chem. Rev. 2003, 238, 383-412; eP. R. Varadwaj, A. Varadwaj, B.-Y. Jin, PCCP 2014, 16, 17238-17252; fF. Cortés-Guzmán, R. F. Bader, Coord. Chem. Rev. 2005, 249, 633-662; gI. Pápai, J. Mascetti, R. Fournier, J. Phys. Chem. A 1997, 101, 4465-4471; hL. J. Farrugia, H. M. Senn, J. Phys. Chem. $A$ 2010, 114, 13418-13433; iL. J. Farrugia, C. Evans, H. M. Senn, M. M. Hänninen, R. Sillanpää, Organometallics 2012, 31, 2559-2570; jC. F. Matta, R. J. Boyd, in The Quantum Theory of Atoms in Molecules, Wiley-VCH Verlag GmbH \& Co. KGaA, 2007, pp. 1-34; kS. Grabowsky, D. Jayatilaka, R. F. Fink, T. Schirmeister, B. Engels, Z. Anorg. Allg. Chem. 2013, 639, 1905-1921; IN. S. Lambic, R. D. Sommer, E. A. Ison, J. Am. Chem. Soc 2016, 138, 4832-4842; mY. Lee, J. C. Peters, J. Am. Chem. Soc. 2011, 133, 4438-4446; nS. T. Howard, O. Lamarche, J. Phys. Org. Chem. 2003, 16, 133141; oR. B. Viana, A. R. Guimarães, A. R. de Souza, A. B. F. da Silva, J. Mol. Model. 2014, 20, 2074; pC. Gatti, in The Quantum Theory of Atoms in Molecules, Wiley-VCH Verlag GmbH \& Co. KGaA, 2007, pp. 163-206; qL. J. Farrugia, P. R. Mallinson, B. Stewart, Acta Cryst. B. 2003, 59, 234-247; rD. J. Duarte, M. S. Miranda, J. C. E. da Silva, J. F. Liebman, Struct. Chem. 2016, 27, 663-670; sJ. Dobado, J. M. Molina, R. Uggla, M. R. Sundberg, Inorg. Chem. 2000, 39, 2831-2836; tS. Pillet, M. Souhassou, C. Mathonière, C. Lecomte, J. Am. Chem. Soc. 2004, 126, 1219-1228; uR. Bianchi, G. Gervasio, D. Marabello, Inorg. Chem. 2000, 39, 2360-2366; vM. A. Alvarez, M. E. García, D. García-Vivó, S. Menéndez, M. A. Ruiz, Organometallics 2012, 31, 7181-7190; wM. A. Alvarez, I. Amor, M. E. García, D. García-Vivó, M. A. Ruiz, J. Suárez, Organometallics 2010, 29, 4384-4395; xM. E. García, D. García-Vivó, S. Melón, M. A. Ruiz, C. Graiff, A. Tiripicchio, Inorg. Chem. 2009, 48, 9282-9293; yM. A. Alvarez, M. E. García, D. García-Vivó, M. E. Martínez, M. A. Ruiz, Organometallics 2011, 30, 2189-2199; zG. F. Caramori, G. Frenking, Theor. Chem. Acc. 2008, 120, 351-361; aaL. J. Farrugia, C. Evans, J. Phys. Chem. A 2005, 109, 8834-8848; abJ. Malecki, T. Groń, H. Duda, Polyhedron 2012, 31, 319-331; acJ. N. Latosińska, J. Seliger, V. Žagar, D. V. Burchardt, J. Mol. Model. 2012, 18, 11-26; adD. Heift, Z. Benkő, H. Grützmacher, A. R. Jupp, J. M. Goicoechea, Chem. Sci. 2015, 6, 4017-4024; aeO. Sizova, A. Y. Sokolov, L. Skripnikov, Russ. J. Coord. Chem. 2007, 33, 800-808; afN. Deora, PhD thesis, Virginia Tech 2010; agG. M. Chiarella, C. Feng, C. A. Murillo, Q. Zhao, Inorg. Chem. 2016, 55, 9471-9481; ahK. K. Pandey, A. Lledós, J. Organomet. Chem. 2010, 695, 206-214; aiZ. S. Marković, J. M. Dimitrić-Marković, D. Milenković, N. Filipović, Monatsh. Chem. 2011, 142, 145-152; ajV. Franco, J. Manuel, G. Schnakenburg, T. Sasamori, A. Espinosa Ferao, R. Streubel, Chem. Eur. J. 2015, 21, 9650-9655; akF. Teixeira, R. Mosquera, A. Melo, C. Freire, M. N. I. DS Cordeiro, Inorg. Chem. 2016, 55, 3653-3662; alH. Raissi, A. Jalbout, M. Nasseria, M. Yoosefian, H. Ghiassi, A. Hameed, Int. J. Quantum Chem 2008, 108, 1444-1451; amS. P. Thomas, M. S. Pavan, T. G. Row, Chem. Commun. 2014, 50, 49-51.

[13] aD. Bykov, PhD thesis, Bonn University 2013; bV. Barbieri, PhD thesis, Milan University 2012 ; cN. S. Emel'yanova, N. y. Y. Shmatko, N. A. Sanina, S. M. Aldoshin, Comput. Theor. Chem. 2015, 1060, 1-9; dC.-R. Lee, I.-J. Hsu, H.-T. Chen, G.-H. Lee, Y. Wang, C.R. Chim. 2012, 15, 237-249; eV. Shlyapochnikov, M. Tafipolsky, I. Tokmakov, E. Baskir, O. Anikin, Y. A. Strelenko, O. Luk'yanov, V. Tartakovsky, J. Mol. Struct. 2001, 559, 147-166; fS. Berski, A. J. Gordon, Chem. Phys. Lett. 2012, 525, 24-31; gS. Berski, A. J. Gordon, J. Chem. Phys. 2011, 135, 094303; hS. Berski, A. J. Gordon, Z. Latajka, J. Chem. Phys. 2013, 138, 134313; iS. V. Shishkina, A. I. Slabko, S. Berski, Z. Latajka, O. V. Shishkin, J. Chem. Phys. 2013, 139, 124308; jV. G. Tsirelson, A. V. Shishkina, A. I. Stash, S. Parsons, Acta Cryst. B. 2009, 65, 647-658; kA. J. Rybarczyk-Pirek, M. Lukomska-Rogala, S. Wojtulewski, M. Palusiak, Cryst. Growth Des. 2015, 15, 5802-5815; IF. Hill, G. Gibbs, M. Boisen Jr, Phys. Chem. Miner. 
1997, 24, 582-596; mC. Trujillo, G. Sánchez-Sanz, I. Alkorta, J. Elguero, New J. Chem. 2015, 39, 67916802; nD. Chesnut, J. Am. Chem. Soc. 1998, 120, 10504-10510; oM. A. Samsonov, PhD thesis, Nizhny Novgorod State Technical University 2017; pL. K. Harper, A. L. Shoaf, C. A. Bayse, Chem PhysChem 2015, 16, 3886-3892; qÁ. Vega-Vega, A. Largo, P. Redondo, C. Barrientos, ACS Earth Space Chem. 2017, 1, 158-167; rE. A. Zhurova, A. I. Stash, V. G. Tsirelson, V. V. Zhurov, E. V. Bartashevich, V. A. Potemkin, A. A. Pinkerton, J. Am. Chem. Soc. 2006, 128, 14728-14734; sA. Y. Rogachev, P. Burger, PCCP 2012, 14, 1985-2000; tM. Sajid, A. Stute, A. J. P. Cardenas, B. J. Culotta, J. A. Hepperle, T. H. Warren, B. Schirmer, S. Grimme, A. Studer, C. G. Daniliuc, J. Am. Chem. Soc. 2012, 134, 1015610168; uM. J. Chalkley, J. C. Peters, Angew. Chem. 2016, 128, 12174-12177; vI. Nieto-López, L. Hernández-García, J. Bonilla-Cruz, M. Sanchez, J. Mol. Model. 2014, 20, 2149; wB. Wang, Y.-M. Lee, W.-Y. Tcho, S. Tussupbayev, S.-T. Kim, Y. Kim, M. S. Seo, K.-B. Cho, Y. Dede, B. C. Keegan, Nat. Commun. 2017, 8, 14839; xM. Nahali, F. Gobal, Mol. Phys. 2011, 109, 229-237; yM. Sander, A. Raj, O. Inderwildi, M. Kraft, S. Kureti, H. Bockhorn, Carbon 2009, 47, 866-875; zR. Salzmann, M. Wojdelski, M. McMahon, R. H. Havlin, E. Oldfield, J. Am. Chem. Soc. 1998, 120, 1349-1356; aaG. F. Caramori, A. G. Kunitz, D. F. Coimbra, L. C. Garcia, D. E. Fonseca, J. Brazilian Chem. Soc. 2013, 24, 1487-1496; abG. Wu, J. Zhu, X. Mo, R. Wang, V. Terskikh, J. Am. Chem. Soc. 2010, 132, 5143-5155; acM. L. Kuznetsov, V. Y. Kukushkin, Dalton Trans. 2017, 46, 786-802; adO. Sizova, N. Ivanova, O. Lyubimova, V. Sizov, Russ. J. Coord. Chem. 2007, 33, 523-529.

[14] aV. G. Avakyan, V. F. Sidorkin, E. F. Belogolova, S. L. Guselnikov, L. E. Gusel'nikov, Organometallics 2006, 25, 6007-6013; bM. Prencipe, M. Tribaudino, F. Nestola, Phys. Chem. Miner. 2003, 30, 606-614; cR. Bianchi, A. Forni, F. Cámara, R. Oberti, H. Ohashi, Phys. Chem. Miner. 2007, 34, 519-527; dR. Bianchi, A. Forni, R. Oberti, Phys. Chem. Miner. 2005, 32, 638-645; eR. Müller, PhD thesis, Technischen Universität Berlin 2014; fN. W. Mitzel, K. Vojinović, R. Fröhlich, T. Foerster, H. E. Robertson, K. B. Borisenko, D. W. Rankin, J. Am. Chem. Soc. 2005, 127, 13705-13713; gQ. Wei, Q. Li, J. Cheng, W. Li, H.-B. Li, RSC Advances 2016, 6, 79245-79253; hS. U. Ahmad, T. Szilvási, E. Irran, S. Inoue, J. Am. Chem. Soc. 2015, 137, 5828-5836; iN. Kocher, PhD thesis, Bayerischen JuliusMaximilians-Universität Würzburg 2003; jV. F. Sidorkin, E. P. Doronina, Organometallics 2009, 28, 5305-5315; kR. J. Gillespie, S. A. Johnson, Inorg. Chem. 1997, 36, 3031-3039; IM. Nechaev, T. Aksamentova, M. Voronkov, N. Chipanina, O. Trofimova, Y. I. Bolgova, V. Turchaninov, Russ. J. Gen. Chem. 2009, 79, 1086-1089; mN. N. Chipanina, N. F. Lazareva, T. N. Aksamentova, A. Y. Nikonov, B. A. Shainyan, Organometallics 2014, 33, 2641-2652; nN. N. Chipanina, N. F. Lazareva, L. P. Oznobikhina, I. M. Lazarev, B. A. Shainyan, Comput. Theor. Chem. 2015, 1070, 162-173; oJ. Dillen, J. Phys. Chem. A 2004, 108, 4971-4977; pM. K. Hazrati, N. L. Hadipour, Phys. Lett. A 2016, 380, 937941; qY.-t. Liu, X. Wang, X.-y. Liu, X.-p. Li, Y.-q. Ji, Chem. Res. Chin. Univ. 2013, 29, 351-354; rJ. D. Epping, S. Yao, M. Karni, Y. Apeloig, M. Driess, J. Am. Chem. Soc. 2010, 132, 5443-5455; sD. CortésArriagada, A. Toro-Labbé, RSC Advances 2016, 6, 28500-28511; tC. M. Marchand, U. Pidun, G. Frenking, H. Grützmacher, J. Am. Chem. Soc. 1997, 119, 11078-11085; uM. Liu, Q. Li, J. Cheng, W. Li, H.-B. Li, J. Chem. Phys. 2016, 145, 224310; vJ.-F. Boily, J. Phys. Chem. A 2002, 106, 4718-4724; wI. Alkorta, I. Rozas, J. Elguero, J. Phys. Chem. A 2001, 105, 743-749; xR. Rodriguez, D. Gau, T. Troadec, N. Saffon-Merceron, V. Branchadell, A. Baceiredo, T. Kato, Angew. Chem. 2013, 125, 9150-9153; yY. Xiong, S. Yao, R. Müller, M. Kaupp, M. Driess, Nat. Chem. 2010, 2, 577-580; zS. S. Sen, Angew. Chem. Int. Ed. 2014, 53, 8820-8822; aaR. Rodriguez, D. Gau, J. Saouli, A. Baceiredo, N. Saffon-Merceron, V. Branchadell, T. Kato, Angew. Chem. 2017, 129, 3993-3997; abM. E. Alikhani, J. Chem. Soc., Faraday Trans. 1997, 93, 3305-3308; acE. P. Doronina, V. F. Sidorkin, N. F. Lazareva, J. Phys. Chem. A 2015, 119, 3663-3673; adP. Suman, S. Janardan, M. Y. Lone, P. Jha, K. Vijayakrishna, A. Sivaramakrishna, Polyhedron 2015, 99, 266-274; aeI. Beletskii, A. Grebenyuk, V. Klimenko, Theor. Exp. Chem. 1995, 30, 187-190; afY. I. Gorlov, V. Zaets, M. Terets, A. Chuiko, Theor. Exp. Chem. 1991, 27, 17-19; agY. I. Gorlov, V. Zaets, A. Chuiko, Theor. Exp. Chem. 1989, 24, 516-526.

[15] aM. Alonso, M. A. Alvarez, M. E. García, D. García-Vivó, M. A. Ruiz, Dalton Trans. 2014, 43, 1607416083; bJ. Pérez, E. Pérez, R. Carrascosa, A. Espinosa, L. García, J. L. Serrano, J. Mol. Struct. 2010, 968, 52-58; cJ. M. V. Franco, T. Sasamori, G. Schnakenburg, A. E. Ferao, R. Streubel, Chem. Commun. 2015, 51, 3878-3881; dG. Balázs, J. C. Green, D. M. P. Mingos, Eur. J. Inorg. Chem. 2007, 2007, 2443-2453; eM. Gall, M. Breza, J. Mol. Struct. THEOCHEM 2009, 894, 32-35; fE. Aubert, PhD thesis, Université Henri Poincaré - Nancy I 2003; gN. Y. Acelas, D. López, F. Mondragón, W. Tiznado, E. Flórez, J. Mol. Model. 2013, 19, 2057-2067; hC. F. Matta, A. A. Arabi, T. A. Keith, J. Phys. Chem. A 2007, 111, 8864-8872; iA. Mermer, T. Lis, P. Starynowicz, RSC Advances 2015, 5, 96623-96638; jA. Hernandez-Laguna, C. Sainz-Diaz, Y. Smeyers, J. De Paz, E. Galvez-Ruano, J. Phys. Chem. 1994, 98, 1109-1116; kD. Hou, Z. Yang, S. Pan, J. Alloys Compd. 2017, 706, 589-595; IM. A. Austen, PhD thesis, Dalhousie University 2003; mI. Alkorta, J. Elguero, S. J. Grabowski, PCCP 2015, 17, 3261-3272; nA. A. Restrepo-Cossio, H. Cano, F. Mari, C. A. Gonzalez, Heteroat. Chem 1997, 8, 557-569; oM. Esseffar, A. Luna, O. Mo, M. Yanez, J. Phys. Chem. 1994, 98, 8679-8686; pD. Mandal, K. Sen, A. K. Das, J. 
Phys. Chem. A 2012, 116, 8382-8396; qA. T. Carvalho, A. C. O'Donoghue, D. R. Hodgson, S. C. Kamerlin, Org. Biom. Chem. 2015, 13, 5391-5398.

[16] aA. Poater Teixidor, PhD thesis, Universitat de Girona 2006; bC. J. Cramer, W. B. Tolman, K. H. Theopold, A. L. Rheingold, PNAS 2003, 100, 3635-3640; cM. F. Qayyum, R. Sarangi, K. Fujisawa, T. D. P. Stack, K. D. Karlin, K. O. Hodgson, B. Hedman, E. I. Solomon, J. Am. Chem. Soc. 2013, 135, 17417-17431; dC. Du, H. Lin, B. Lin, Z. Ma, T. Hou, J. Tang, Y. Li, J. Mater. Chem. A 2015, 3, 2311323119; eA. F. Cozzolino, D. Tofan, C. C. Cummins, M. Temprado, T. D. Palluccio, E. V. RybakAkimova, S. Majumdar, X. Cai, B. Captain, C. D. Hoff, J. Am. Chem. Soc. 2012, 134, 18249-18252; fC. Rovira, K. Kunc, J. Hutter, P. Ballone, M. Parrinello, J. Phys. Chem. A 1997, 101, 8914-8925; gV. Luana, A. Costales, P. Mori-Sanchez, A. M. Pendás, J. Phys. Chem. B 2003, 107, 4912-4921; hG. V. Gibbs, R. Downs, D. F. Cox, N. L. Ross, M. Boisen, K. M. Rosso, J. Phys. Chem. A 2008, 112, 36933699; iG. Gibbs, M. Boisen, K. Rosso, D. Teter, M. Bukowinski, J. Phys. Chem. B 2000, 104, 1053410542; jG. Gibbs, O. Tamada, M. Boisen, F. Hill, Am. Mineral. 1999, 84, 435-446; kA. Pakiari, K. Eskandari, J. Mol. Struct. THEOCHEM 2007, 806, 1-7; IC. Sharma, A. K. Singh, J. Joy, E. D. Jemmis, S. K. Awasthi, RSC Advances 2016, 6, 91689-91693; mV. R. Hathwar, M. K. Thomsen, M. A. H. Mamakhel, М. Ø. Filsø, J. Overgaard, B. B. Iversen, J. Phys. Chem. A 2016, 120, 7510-7518; nX. Fradera, M. Solà, J. Comput. Chem. 2002, 23, 1347-1356; oY. Yang, J. Phys. Chem. A 2012, 116, 1015010159; pD. E. Trujillo-González, M. C. Ramírez-Romero, J. I. Rodríguez, E. A. Uribe, Chem. Phys. Lett. 2016, 649, 103-110; qE. A. Zhurova, A. Martin, A. A. Pinkerton, J. Am. Chem. Soc. 2002, 124, 8741-8750; rY. V. Nelyubina, K. A. Lyssenko, Chem. Eur. J. 2012, 18, 12633-12636.

[17] aS. Kundu, C. Mohapatra, P. P. Samuel, J. Kretsch, M. Walawalkar, R. Herbst-Irmer, D. Stalke, S. De, D. Koley, H. W. Roesky, Chem. Commun. 2017, 53, 192-195; bQ. Tang, Q. Li, Comput. Theor. Chem. 2014, 1050, 51-57; cH. Xu, J. Cheng, X. Yang, Z. Liu, X. Bo, Q. Li, RSC Advances 2017, 7, 21713-21720; dM. D. Esrafili, F. Mohammadian-Sabet, Mol. Phys. 2016, 114, 83-91; eM. D. Esrafili, F. Mohammadian-Sabet, Mol. Phys. 2016, 114, 1528-1538; fM. Liu, L. Yang, Q. Li, W. Li, J. Cheng, B. Xiao, X. Yu, J. Mol. Model. 2016, 22, 192; gM. Marín-Luna, I. Alkorta, J. Elguero, J. Organomet. Chem. 2015, 794, 206-215; hM. Marín-Luna, I. Alkorta, J. Elguero, Theor. Chem. Acc. 2017, 136, 41; iS. J. Grabowski, Crystals 2017, 7, 43; jY. Li, H. Zhu, D. M. Andrada, G. Frenking, H. W. Roesky, Chem. Commun. 2014, 50, 4628-4630; kT. Müller, Y. Apeloig, I. Hemme, U. Klingebiel, M. Noltemeyer, J. Organomet. Chem. 1995, 494, 133-142; IM. Novak, L. Dostál, M. Alonso, F. De Proft, A. Rưžička, A. Lyčka, R. Jambor, Chem. Eur. J. 2014, 20, 2542-2550; mY. Xiong, S. Yao, S. Inoue, E. Irran, M. Driess, Angew. Chem. Int. Ed. 2012, 51, 10074-10077; nU. Burckhardt, G. L. Casty, T. D. Tilley, T. K. Woo, U. Rothlisberger, Organometallics 2000, 19, 3830-3841; oY. Yang, J. Phys. Chem. A 2010, 114, 13257-13267; pD. Dhara, T. Vijayakanth, M. K. Barman, K. P. K. Naik, N. Chrysochos, C. B. Yildiz, R. Boomishankar, C. Schulzke, V. Chandrasekhar, A. Jana, Chem. Commun. 2017, 53, 85928595; qT. Ochiai, D. Franz, S. Inoue, Chem. Soc. Rev. 2016, 45, 6327-6344; rS. Inoue, K. Leszczyńska, Angew. Chem. 2012, 124, 8717-8721; sP. Jutzi, Chem. Eur. J. 2014, 20, 9192-9207; tV. G. Avakyan, E. P. Doronina, S. L. Guselnikov, V. F. Sidorkin, J. Organomet. Chem. 2011, 696, 1969-1974; uM. S. Gordon, M. T. Carroll, J. H. Jensen, L. P. Davis, L. W. Burggraf, R. M. Guidry, Organometallics 1991, 10, 2657-2660; vG. Csonka, P. Hencsei, J. Organomet. Chem. 1993, 446, 99-106; wG. Csonka, P. Hencsei, J. Mol. Struct. THEOCHEM 1993, 283, 251-259; xV. F. Sidorkin, E. F. Belogolova, E. P. Doronina, PCCP 2015, 17, 26225-26237; yV. Romanovs, V. Sidorkin, E. Belogolova, V. Jouikov, Dalton Trans. 2017; zG. W. Fester, J. r. Wagler, E. Brendler, U. Böhme, D. Gerlach, E. Kroke, J. Am. Chem. Soc. 2009, 131, 6855-6864.

[18] aJ. Cioslowski, S. T. Mixon, J. Am. Chem. Soc. 1991, 113, 4142-4145; bY. S. Won, Y. S. Kim, T. J. Anderson, L. L. Reitfort, I. Ghiviriga, L. McElwee-White, J. Am. Chem. Soc. 2006, 128, 13781-13788; cC. Cabezas, C. Barrientos, A. Largo, J.-C. Guillemin, J. Alonso, PCCP 2016, 18, 28538-28547; dA. A. Melekhova, A. S. Novikov, N. A. Bokach, M. S. Avdonceva, V. Y. Kukushkin, Inorg. Chim. Acta 2016, 450, 140-145; eZ. Setifi, K. V. Domasevitch, F. Setifi, P. Mach, S. W. Ng, V. Petř́iček, M. Dušek, Acta Cryst. C. 2013, 69, 1351-1356; fD. Kratzert, PhD thesis, Göttingen 2013; gD. E. Hibbs, C. J. Austin-Woods, J. A. Platts, J. Overgaard, P. Turner, Chem. Eur. J. 2003, 9, 1075-1084; hJ. A. Dobado, R. Uggla, M. R. Sundberg, J. Molina, Dalton Trans. 1999, 489-496; iE. Espinosa, E. Molins, C. Lecomte, Phys. Rev. B. 1997, 56, 1820; jL. Álvarez-Rodríguez, J. Brugos, J. A. Cabeza, P. GarcíaÁlvarez, E. Pérez-Carreño, D. Polo, Chem. Commun. 2017, 53, 893-896; kX.-H. Li, R.-Z. Zhang, H.-L. Cui, Y.-L. Yong, Chem. Heterocycl. Compd. 2015, 51, 153-158; IC. Bo, J. M. Poblet, M. Bénard, Chem. Phys. Lett. 1990, 169, 89-96; mH. Jansen, F. B. Läng, J. C. Slootweg, A. W. Ehlers, M. Lutz, K. Lammertsma, H. Grützmacher, Angew. Chem. 2010, 122, 5617-5620; nZ. Azizi, M. Ghambarian, M. A. Rezaei, M. Ghashghaee, Aust. J. Chem. 2015, 68, 1438-1445; oP. J. Byrne, PhD thesis, University of St Andrews 2009; pM. Liu, Q. Li, W. Li, J. Cheng, Struct. Chem. 2017, 28, 823-831; qB. Freitag, C. A. Fischer, J. Penafiel, G. Ballmann, H. Elsen, C. Färber, D. F. Piesik, S. Harder, Dalton Trans. 2017, 46, 11192-11200 ; rA. Bauzá, T. J. Mooibroek, A. Frontera, Chemical Record 2016, 16, 473-487; sA. 
Ariafard, M. D. Asli, H. Aghabozorg, H. Aghabozorg, M. Monajjemi, J. Mol. Struct. THEOCHEM 2003, 636, 49-56; tJ. M. V. Franco, G. Schnakenburg, A. E. Ferao, R. Streubel, Dalton Trans. 2016, 45, 13951-13956; uG. von Frantzius, A. E. Ferao, R. Streubel, Chem. Sci. 2013, 4, 4309-4322; vD. Mani, E. Arunan, PCCP 2013, 15, 14377-14383; wS. J. Grabowski, PCCP 2014, 16, 1824-1834; xM. D. Esrafili, N. Mohammadirad, M. Solimannejad, Chem. Phys. Lett. 2015, 628, 16-20.

[19] aC. L. Firme, S. E. Galembeck, O. Antunes, P. M. Esteves, J. Brazilian Chem. Soc. 2007, 18, 13971404; bK. Albahily, PhD thesis, Ottawa-Carleton Chemistry Institute 2011; cC. Lacaze-Dufaure, T. Mineva, N. Russo, J. Comput. Chem. 2001, 22, 1557-1564; dT. Lu, F. Chen, J. Phys. Chem. A 2013, 117, 3100-3108; eM. Tafipolsky, W. Scherer, K. Öfele, G. Artus, B. Pedersen, W. A. Herrmann, G. S. McGrady, J. Am. Chem. Soc. 2002, 124, 5865-5880; fL. J. Farrugia, C. Evans, D. Lentz, M. Roemer, J. Am. Chem. Soc. 2008, 131, 1251-1268; gA. I. Baranov, M. Kohout, J. Comput. Chem. 2011, 32, 20642076; hV. César, L. C. Misal Castro, T. Dombray, J.-B. Sortais, C. Darcel, S. p. Labat, K. Miqueu, J.M. Sotiropoulos, R. m. Brousses, N. Lugan, Organometallics 2013, 32, 4643-4655; iS. Scheins, M. Messerschmidt, M. Gembicky, M. Pitak, A. Volkov, P. Coppens, B. G. Harvey, G. C. Turpin, A. M. Arif, R. D. Ernst, J. Am. Chem. Soc. 2009, 131, 6154-6160; jS. K. Brayshaw, E. L. Sceats, J. C. Green, A. S. Weller, PNAS 2007, 104, 6921-6926; kC. Gatti, Z. Kristallogr. 2005, 220, 399-457; lH. Nuss, N. Claiser, S. Pillet, N. Lugan, E. Despagnet-Ayoub, M. Etienne, C. Lecomte, Dalton Trans. 2012, 41, 6598-6601; mR. Destro, R. Soave, M. Barzaghi, L. Lo Presti, Chem. Eur. J. 2005, 11, 4621-4634; nC. L. Firme, O. Antunes, P. M. Esteves, J. Brazilian Chem. Soc. 2009, 20, 543-548; oC. L. Firme, O. Antunes, P. M. Esteves, Chem. Phys. Lett. 2009, 468, 129-133; pP. Popelier, G. Logothetis, J. Organomet. Chem. 1998, 555, 101-111; qJ. Henn, D. Leusser, D. Stalke, J. Comput. Chem. 2007, 28, 2317-2324; rF. Feixas, E. Matito, J. Poater, M. Sola, J. Phys. Chem. A 2011, 115, 13104-13113; sK. Remya, C. H. Suresh, PCCP 2015, 17, $27035-27044$.

[20] aM. Ray, Y. Nakao, H. Sato, H. Sakaba, S. Sakaki, Organometallics 2008, 28, 65-73; bW. Scherer, P. Sirsch, M. Grosche, M. Spiegler, S. A. Mason, M. G. Gardiner, Chem. Commun. 2001, 2072-2073; cH. Ott, PhD thesis, Georg-August-Universität Göttingen 2009; dL. Olsson, D. Cremer, Chem. Phys. Lett. 1993, 215, 433-443; eD. Stalke, in The Chemical Bond I, Springer, 2016, pp. 57-88; fC. Mohapatra, S. Kundu, A. N. Paesch, R. Herbst-Irmer, D. Stalke, D. M. Andrada, G. Frenking, H. W. Roesky, J. Am. Chem. Soc. 2016, 138, 10429-10432; gE. D. Jemmis, R. Saradha, A. Saieswari, J. Elambalassery, Indian J. Chem. 2003, 42, 2382-2391; hC. B. Yıldı, A. Azizoglu, Struct. Chem. 2012, 23, 1777-1784; iL. C. Pavelka, M. A. Hanson, V. N. Staroverov, K. M. Baines, Can. J. Chem. 2014, 93, 134-142; jM. Bendikov, Y. Apeloig, S. Bukalov, I. Garbuzova, L. Leites, J. Phys. Chem. A 2002, 106, 4880-4885; kX. F. Duan, L. W. Burggraf, L. Huang, Molecules 2013, 18, 8591-8606; ID. Lutters, C. Severin, M. Schmidtmann, T. Müller, J. Am. Chem. Soc 2016, 138, 6061-6067; mY. Wang, M. Chen, Y. Xie, P. Wei, H. F. Schaefer III, G. H. Robinson, J. Am. Chem. Soc. 2015, 137, 8396-8399; nP. V. Bharatam, R. Moudgil, D. Kaur, Organometallics 2002, 21, 3683-3690; oJ.-H. Sheu, M.-D. Su, Organometallics 2012, 31, 3101-3112; pJ.-j. Sui, J. Xu, Y.-h. Ding, Dalton Trans. 2016, 45, 56-60; qA. E. Ketvirtis, D. K. Bohme, A. C. Hopkinson, J. Phys. Chem. A 1999, 103, 11161-11171; rI. Billas, C. Massobrio, M. Boero, M. Parrinello, W. Branz, F. Tast, N. Malinowski, M. Heinebrodt, T. Martin, J. Chem. Phys. 1999, 111, 6787-6796; sJ. M. Rintelman, M. S. Gordon, J. Chem. Phys. 2001, 115, 1795-1803; tB. Niepötter, PhD thesis, Georg-August University School of Science, Göttingen 2015; uC. Eisenhut, T. Szilvási, G. Dübek, N. C. Breit, S. Inoue, Inorg. Chem. 2017, 56, 10061-10069; vS. Roy, K. C. Mondal, T. Mondal, D. Koley, B. Dittrich, H. W. Roesky, Dalton Trans. 2015, 44, 19942-19947; wD. Lutters, A. Merk, M. Schmidtmann, T. Müller, Inorg. Chem. 2016, 55, 9026-9032; xV. Y. Lee, O. A. Gapurenko, Y. Ito, T. Meguro, H. Sugasawa, A. Sekiguchi, R. M. Minyaev, V. I. Minkin, R. H. Herber, H. Gornitzka, Organometallics 2016, 35, 346-356; yG. Martinez-Guajardo, Z. Gómez-Saldoval, D. F. Jana, P. Calaminici, C. Corminboeuf, G. Merino, PCCP 2011, 13, 20615-20619; zP. Jutzi, K. Leszczyńska, B. Neumann, W. W. Schoeller, H. G. Stammler, Angew. Chem. Int. Ed. 2009, 48, 2596-2599; aaV. G. Avakyan, S. L. Guselnikov, V. F. Sidorkin, E. P. Doronina, L. E. Gusel'nikov, Organometallics 2009, 28, 978-989; abP. Ghana, M. I. Arz, U. Das, G. Schnakenburg, A. C. Filippou, Angew. Chem. Int. Ed. 2015, 54, 9980-9985; acF. Shojaie, Comput. Theor. Chem. 2017, 1114, 55-64; adD. Gau, T. Kato, N. Saffon-Merceron, A. De Cózar, F. P. Cossío, A. Baceiredo, Angew. Chem. 2010, 122, 6735-6738; aeD. Gau, R. Rodriguez, T. Kato, N. Saffon-Merceron, A. de Cózar, F. P. Cossío, A. Baceiredo, Angew. Chem. 2011, 123, 1124-1128; afM. Marín-Luna, I. Alkorta, J. Elguero, J. Phys. Chem. A 2016, 120, 648-656; agS.-W. Tang, F.-D. Wang, Y.-H. Li, F. Wang, S.-B. Yang, H. Sun, Y.-F. Chang, R.-S. Wang, J. Mol. Model. 2013, 19, 5579-5586.

[21] aJ.-W. Zou, M. Huang, G.-X. Hu, Y.-J. Jiang, RSC Advances 2017, 7, 10295-10305; bS. Berski, Z. Ciunik, K. Drabent, Z. Latajka, J. Panek, J. Phys. Chem. B 2004, 108, 12327-12332; cJ. Joy, E. D. Jemmis, K. Vidya, Faraday Discuss. 2015, 177, 33-50; dV. Oliveira, E. Kraka, D. Cremer, Inorg. Chem. 2017, 56, 488-502; eH. Xu, J. Cheng, Q. Li, W. Li, Mol. Phys. 2016, 114, 3643-3649; fD. C. 
Georgiou, P. Butler, E. C. Browne, D. J. Wilson, J. L. Dutton, Aust. J. Chem. 2013, 66, 1179-1188; gJ. J. Queralt, J. Andrés, M. Canle, J. H. Cobas, J. A. Santaballa, J. R. Sambrano, Chem. Phys. 2002, 280, 1-14; hA. Walczak, B. Brycki, M. Kaczmarek, O. K. Poleshchuk, M. Ostafin, B. Nogaj, Chem. Phys. 2006, 325, 429-436; iA. Petelski, D. J. Duarte, S. Pamies, N. Peruchena, G. Sosa, Theor. Chem. Acc. 2016, 135, 65; jR. D. Parra, Comput. Theor. Chem. 2012, 998, 183-192; kE. Bartashevich, E. Troitskaya, Á. M. Pendás, V. Tsirelson, Comput. Theor. Chem. 2015, 1053, 229-237; IJ. Andrés, X. L. Armesto, M. Canle, M. V. García, D. R. Ramos, J. A. Santaballa, Org. Biom. Chem. 2003, 1, 43234328; mM. D. Esrafili, P. Mousavian, Chem. Phys. Lett. 2017, 678, 275-282.

[22] aV. Luana, A. M. Pendas, A. Costales, G. A. Carriedo, F. J. García-Alonso, J. Phys. Chem. A 2001, 105, 5280-5291; bK. K. Pandey, P. Tiwari, P. Patidar, J. Phys. Chem. A 2012, 116, 11753-11762; cK. K. Pandey, P. Tiwari, P. Patidar, J. Organomet. Chem. 2013, 740, 135-140; dK. K. Pandey, P. Tiwari, P. Patidar, S. K. Patidar, R. Vishwakarma, P. K. Bariya, J. Organomet. Chem. 2014, 751, 781-787; eK. K. Pandey, R. Vishwakarma, J. Organomet. Chem. 2016, 813, 84-94; fI. Alkorta, J. Elguero, J. E. Del Bene, J. Phys. Chem. A 2013, 117, 10497-10503; gA. B. Chaplin, J. A. Harrison, P. J. Dyson, Inorg. Chem. 2005, 44, 8407-8417; hJ. E. Del Bene, I. Alkorta, J. Elguero, J. Phys. Chem. A 2015, 119, 1170111710; iZ. Benkő, R. Streubel, L. Nyulászi, Dalton Trans. 2006, 4321-4327; jÁ. Sánchez-González, S. Melchor, J. A. Dobado, B. Silvi, J. Andrés, J. Phys. Chem. A 2011, 115, 8316-8326; kJ. E. Del Bene, I. Alkorta, G. Sánchez-Sanz, J. Elguero, J. Phys. Chem. A 2012, 116, 9205-9213; IV. Jancik, F. Cortés-Guzmán, R. Herbst-Irmer, D. Matínez-Otero, Chem. Eur. J. 2017, 23, 6964-6968; mM. Calichman, A. Derecskei-Kovacs, C. W. Allen, Inorg. Chem. 2007, 46, 2011-2016; nK. Gholivand, H. R. Mahzouni, M. D. Esrafili, Dalton Trans. 2012, 41, 1597-1608; oT. L. Chan, Z. Xie, Chem. Commun. 2016, 52, 7280-7283; pJ. E. Del Bene, I. Alkorta, J. Elguero, G. Sánchez-Sanz, J. Phys. Chem. A 2017, 121, 1362-1370; qL. Ascherl, A. Nordheider, K. S. A. Arachchige, D. B. Cordes, K. Karaghiosoff, M. Bühl, A. M. Slawin, J. D. Woollins, Chem. Commun. 2014, 50, 6214-6216; rM. Demange, L. Boubekeur, A. Auffrant, N. Mézailles, L. Ricard, X. Le Goff, P. Le Floch, New J. Chem. 2006, 30, 1745-1754; sL. F. Ji, A. Y. Li, Z. Z. Li, Z. X. Ge, J. Mol. Model. 2015, $22,1$.

[23] aM. W. Wong, K. B. Wiberg, J. Am. Chem. Soc. 1992, 114, 7527-7535; bK. Haupa, A. Bil, Z. Mielke, J. Phys. Chem. A 2015, 119, 10724-10734; cD. Setiawan, E. Kraka, D. Cremer, J. Phys. Chem. A 2015, 119, 9541-9556; dB. L. Tran, R. Thompson, S. Ghosh, X. Gao, C.-H. Chen, M.-H. Baik, D. J. Mindiola, Chem. Commun. 2013, 49, 2768-2770; eK. K. Pandey, J. Coord. Chem. 1990, 22, 307-313; fT. Pasinszki, G. Bazsó, M. Krebsz, G. Tarczay, PCCP 2009, 11, 9458-9467; gA. J. Bridgeman, G. Cavigliasso, L. R. Ireland, J. Rothery, Dalton Trans. 2001, 2095-2108; hS. Feth, PhD thesis, Virginia 1996; iC. M. Breneman, L. W. Weber, Can. J. Chem. 1996, 74, 1271-1282; jL. Lo Presti, A. M. Orlando, L. Loconte, R. Destro, E. Ortoleva, R. Soave, C. Gatti, Cryst. Growth Des. 2014, 14, 4418-4429; kM. D. Esrafili, F. Mohammadian-Sabet, Mol. Phys. 2015, 113, 95-103; IL. M. Azofra, I. Alkorta, S. Scheiner, J. Phys. Chem. A 2015, 119, 535-541; mM. Macernis, J. Chem. 2017, 2017.

[24] aM. F. Lucas, M. C. Michelini, N. Russo, E. Sicilia, J. Chem. Theory Comput. 2008, 4, 397-403; bS. M. Bachrach, J. Phys. Chem. 1989, 93, 7780-7784; cS. M. Bachrach, J. Comput. Chem. 1989, 10, 392-406; dD. Heift, Z. Benkő, H. Grützmacher, Dalton Trans. 2014, 43, 831-840; eA. Doddi, D. Bockfeld, T. Bannenberg, P. G. Jones, M. Tamm, Angew. Chem. 2014, 126, 13786-13790; fL. Liu, D. A. Ruiz, F. Dahcheh, G. Bertrand, Chem. Commun. 2015, 51, 12732-12735; gC. C. Chong, B. Rao, R. Ganguly, Y. Li, R. Kinjo, Inorg. Chem. 2017; hM. Yáñez, J. Phys. Org. Chem. 1998, 11, 678-683; iR. A. Rajagopalan, PhD thesis, University of Regina 2014; jS. M. Bachrach, J. Mol. Struct. THEOCHEM 1992, 255, 207-219; kL. Nyulaszi, T. Veszpremi, J. Reffy, J. Phys. Chem. 1995, 99, 10142-10146; IG. v. Frantzius, A. E. Ferao, R. Streubel, Chem. Sci. 2013, 4, 4309-4322; mS. Samiee, N. Kooti, H. Motamedi, R. W. Gable, F. A. Bagherjeri, Polyhedron 2015, 98, 120-130; nH. Joo, PhD thesis, Auburn University, Alabama 2005.

[25] aP. R. Schreiner, H. P. Reisenauer, J. Romanski, G. Mloston, Angew. Chem. Int. Ed. 2009, 48, 81338136; bH. S. Rzepa, J. Chem. Theory Comput. 2010, 7, 97-102; cC. Guo, N. Hou, C. Wang, J. Mol. Model. 2016, 22, 1-6; dC. Guo, C. Wang, Y.-h. Ding, Struct. Chem. 2014, 25, 1023-1031; eC. Guo, Z. H. Cui, Y. H. Ding, Int. J. Quantum Chem 2013, 113, 2213-2219; fA. M. Masdeu, A. Ruiz, S. Castillon, C. Claver, P. B. Hitchcock, P. A. Chaloner, C. Bo, J. M. Poblet, P. Sarasa, Dalton Trans. 1993, 2689-2696; gA. Vashchenko, A. Abramov, Y. L. Frolov, J. Mol. Struct. THEOCHEM 2002, 594, 107-111; hB. Fresch, F. Remacle, J. Phys. Chem. C 2014, 118, 9790-9800; iC.-R. Lee, L.-Y. Tan, Y. Wang, J. Phys. Chem. Solids 2001, 62, 1613-1628; jI. Sovago, PhD thesis, University of Glasgow 2013; kM. V. Roux, M. Temprado, P. Jiménez, R. Notario, J. S. Chickos, A. F. L. Santos, M. A. Ribeiro da Silva, J. Phys. Chem. A 2007, 111, 11084-11092.

[26] aR. Gaba, M. Bhandari, R. Kakkar, Adv. Mat. Lett. 2013, 4, 769-778; bT. A. Manz, A. E. Fenwick, K. Phomphrai, I. P. Rothwell, K. T. Thomson, Dalton Trans. 2005, 668-674; cV. P. Indrakanti, PhD thesis, The Pennsylvania State University 2009; dE. A. Zhurova, V. G. Tsirelson, Acta Cryst. B. 2002, 58, 567-575; eH. M. I. Pritchard, PhD thesis, University of London 2005; fM. Calatayud, P. Mori- 
Sánchez, A. Beltrán, A. M. Pendás, E. Francisco, J. Andrés, J. Recio, Phys. Rev. B. 2001, 64, 184113; gN. Seriani, J. Phys.: Condens. Matter 2010, 22, 255502; hK. M. Rosso, J. R. Rustad, G. Gibbs, J. Phys. Chem. A 2002, 106, 8133-8138; iM. Srebro, J. Autschbach, Chem. Eur. J. 2013, 19, 12018-12033; jA. K. Phukan, A. K. Guha, Inorg. Chem. 2010, 49, 9884-9890; kA. Robertazzi, J. A. Platts, J. Biol. Inorg. Chem. 2005, 10, 854-866; IM. Zegke, PhD thesis, University of Edinburgh 2015; mD. Duarte Ruíz, Master's thesis, Universidad EAFIT 2015; nS. Dargouthi, S. Boughdiri, B. Tangour, Acta Chim. Slov. 2015, 62, 445-451; oY. Gong, M. Zhou, S. X. Tian, J. Yang, J. Phys. Chem. A 2007, 111, 6127-6130; pA. Marzouk, B. Madebène, M. E. Alikhani, J. Phys. Chem. A 2013, 117, 4462-4471; qW. Jauch, M. Reehuis, Acta Crystallogr. Sect. A: Found. Crystallogr. 2005, 61, 411-417; rR. A. Evarestov, in Quantum Chemistry of Solids, Springer, 2012, pp. 357-488.

[27] aA. J. Bridgeman, G. Cavigliasso, Faraday Discuss. 2003, 124, 239-258; bP. Hejduk, M. Witko, K. Hermann, Top. Catal. 2009, 52, 1105-1115; cJ. K. Bartley, J. S. J. Hargreaves, G. J. Hutchings, J. L. Rico, S. H. Taylor, R. P. K. Wells, D. J. Willock, in Encyclopedia of Catalysis, John Wiley \& Sons, Inc., 2002; dK. Alexopoulos, P. Hejduk, M. Witko, M.-F. Reyniers, G. B. Marin, J. Phys. Chem. C 2010, 114, 3115-3130; eS. Brandán, C. Socolsky, A. B. Altabef, Z. Anorg. Allg. Chem. 2009, 635, 582-592; fC. Das, P. Adak, S. Mondal, R. Sekiya, R. Kuroda, S. I. Gorelsky, S. K. Chattopadhyay, Inorg. Chem. 2014, 53, 11426-11437; gA. Egorova, V. Tsirel'son, Russ. J. Inorg. Chem. 2006, 51, 941-948; hD. Masuzaki, T. Nagata, F. Mafune, J. Phys. Chem. A 2017, 121, 2079-2085.

[28] aM. E. O'Reilly, PhD thesis, University of Florida 2013; bS. A. Brandán, in A Structural and Vibrational Investigation into Chromylazide, Acetate, Perchlorate, and Thiocyanate Compounds, Springer, 2013, pp. 21-56; cM. Sundberg, R. Uggla, R. Sillanpää, K. Zborowski, A. Sánchez-González, J. Matikainen, S. Kaltia, T. Hase, Open Chem. 2010, 8, 486-493; dS. Veliah, K.-h. Xiang, R. Pandey, J. Recio, J. M. Newsam, J. Phys. Chem. B 1998, 102, 1126-1135; eS. A. Brandán, A Structural and Vibrational Study of the Chromyl Chlorosulfate, Fluorosulfate, and Nitrate Compounds, Springer Netherlands, 2012; fM. Torrent Sucarrat, P. Gili Trujillo, M. Duran i Portas, M. Solà i Puig, J. Chem. Phys. 1996, 104, 9499-9510; gC.-C. Wang, T.-H. Tang, Y. Wang, J. Phys. Chem. A 2000, 104, 95669572; hR. J. Gillespie, I. Bytheway, T.-H. Tang, R. F. Bader, Inorg. Chem. 1996, 35, 3954-3963; iZ. Sun, H. F. Schaefer, Y. Xie, Y. Liu, R. Zhong, Theor. Chem. Acc. 2015, 134, 43; jS. A. Brandán, J. Mol. Struct. THEOCHEM 2009, 908, 19-25; kA. B. Altabef, S. Brandán, J. Mol. Struct. 2010, 981, 146-152; IY. Wang, J. Chin. Chem. Soc. 2014, 61, 27-38; mZ. Sun, H. F. Schaefer, Y. Xie, Y. Liu, R. Zhong, J. Comput. Chem. 2014, 35, 998-1009; nA. D. P. Alexiou, C. C. Decandio, S. D. N. Almeida, M. J. P. Ferreira, P. Romoff, R. C. Rocha, J. Coord. Chem. 2017, 1-13.

[29] aM. Jaccob, A. Ansari, B. Pandey, G. Rajaraman, Dalton Trans. 2013, 42, 16518-16526; bP. R. Varadwaj, A. Varadwaj, H. M. Marques, J. Phys. Chem. A 2011, 115, 5592-5601; cD. RutkowskaZbik, R. Tokarz-Sobieraj, M. Witko, J. Chem. Theory Comput. 2007, 3, 914-920; dJ. He, Z.-G. Xu, Y.X. Zeng, X. Xu, L. Yu, Q. Wang, H.-Y. Liu, Acta Phys.-Chim. Sin. 2012, 28, 1658-1664; eA. S. Jepsen, M. Roberson, R. G. Hazell, Chem. Commun. 1998, 1599-1600; fG. V. Gibbs, R. Downs, D. F. Cox, K. M. Rosso, N. L. Ross, A. Kirfel, T. Lippmann, W. Morgenroth, T. D. Crawford, J. Phys. Chem. A 2008, 112, 8811-8823; gR. D. Poulsen, A. Bentien, T. Graber, B. Iversen, Acta Crystallogr. Sect. A: Found. Crystallogr. 2004, 60, 382-389; hC. Lecomte, E. Aubert, V. Legrand, F. Porcher, S. Pillet, B. Guillot, C. Jelsch, Z. Kristallogr. 2005, 220, 373-384; iW. Jauch, M. Reehuis, Phys. Rev. B. 2003, 67, 184420; jQ. Chen, L. Ni, Y.-Y. Hu, J. Yao, W.-L. Zhang, Chin. J. Inorg. Chem. 2014, 30, 1481-1488; kM. J. Javan, Z. A. Tehrani, A. Fattahi, M. M. Hashemi, J. Phys. Org. Chem. 2012, 25, 1198-1209; IH. Kavousi, H. Raissi, A. Rezaeifard, M. Jafarpour, J. Porphyrins Phthalocyanines 2015, 19, 1130-1139; mH. Kavousi, A. Rezaeifard, H. Raissi, M. Jafarpour, J. Porphyrins Phthalocyanines 2015, 19, 651662; nR. A. P. Ribeiro, S. R. de Lazaro, C. Gatti, RSC Advances 2016, 6, 101216-101225.

[30] aP. Chernev, I. Zaharieva, H. Dau, M. Haumann, J. Biol. Chem. 2011, 286, 5368-5374; bW. Yi, L. Yuan, Y. Kun, H. Zhengwen, T. Jing, G. Hong, W. Yong, J. Biol. Inorg. Chem. 2015, 20, 1123-1134; cC. Gómez-Casado, F. Roth-Walter, E. Jensen-Jarolim, A. Díaz-Perales, L. F. Pacios, J. Mol. Graphics Modell. 2013, 45, 111-121; dJ. Overgaard, D. E. Hibbs, E. Rentschler, G. A. Timco, F. K. Larsen, Inorg. Chem. 2003, 42, 7593-7601; eJ. Overgaard, F. K. Larsen, B. Schiøtt, B. B. Iversen, J. Am. Chem. Soc. 2003, 125, 11088-11099; fJ. Overgaard, F. K. Larsen, G. A. Timco, B. B. Iversen, Dalton Trans. 2009, 664-671; gV. Berryman, PhD thesis, Dalhousie University 2015; hX. Yang, Dalton Trans. 2013, 42, 11987-11991; iJ. Murgich, H. J. Franco, J. Phys. Chem. A 2009, 113, 5205-5211; jF. A. La Porta, P. H. Ramos, E. C. de Resende, M. C. Guerreiro, J. O. Giacoppo, T. C. Ramalho, J. R. Sambrano, J. Andrés, E. Longo, Inorg. Chim. Acta 2014, 416, 200-206; kZ. Tang, Y. Wang, P. Zhang, J. Coord. Chem. 2017, 70, 417-430; IY. Ling, C. Mills, R. Weber, L. Yang, Y. Zhang, J. Am. Chem. Soc. 2010, 132, 1583-1591; mS. Kavitha, P. Deepa, M. Karthika, R. Kanakaraju, Polyhedron 2016, 115, 193-203; nB. Shin, K. D. Sutherlin, T. Ohta, T. Ogura, E. I. Solomon, J. Cho, Inorg. Chem. 2016, 55, 1239112399; oJ. Ortega-Castro, J. Frau, R. Casasnovas, D. Fernández, J. Donoso, F. Muñoz, J. Phys. Chem. 
A 2012, 116, 2961-2971; pK. K. Pandey, R. Vishwakarma, S. K. Patidar, P. K. Bariya, Comput. Theor. Chem. 2015, 1051, 137-143.

[31] aJ. Overgaard, J. P. Walsh, V. R. Hathwar, M. R. Jørgensen, C. Hoffman, J. A. Platts, R. Piltz, R. E. Winpenny, Inorg. Chem. 2014, 53, 11531-11539; bP. R. Varadwaj, H. M. Marques, PCCP 2010, 12, 2126-2138; cI. Georgieva, N. Trendafilova, Bulg. Chem. Commun. 2014, 46, 196-202; dJ. J. Lee, G. H. Lee, Y. Wang, Chem. Eur. J. 2002, 8, 1821-1832; eL.-C. Wu, T.-C. Weng, I.-J. Hsu, Y.-H. Liu, G.-H. Lee, J.-F. Lee, Y. Wang, Inorg. Chem. 2013, 52, 11023-11033; fC.-R. Lee, C.-C. Wang, K.-C. Chen, G.H. Lee, Y. Wang, J. Phys. Chem. A 1999, 103, 156-165; gR.-Z. Li, J. Liang, X.-L. Xu, H.-G. Xu, W.-J. Zheng, Chem. Phys. Lett. 2013, 575, 12-17; hH. F. Clausen, PhD thesis, Aarhus University, Denmark 2009; iN. Ghadimi, C. B. Perry, M. A. Fernandes, P. P. Govender, H. M. Marques, Inorg. Chim. Acta 2015, 436, 29-38; jQ.-W. Wang, M. Sun, Y.-N. Wang, X.-F. Qi, X.-M. Li, B. Liu, Chin. J. Struct. Chem. 2015, 34, 393-400; kC. F. Zipp, J. P. Michael, M. A. Fernandes, S. Mathura, C. B. Perry, I. Navizet, P. P. Govender, H. M. Marques, Inorg. Chem. 2014, 53, 4418-4429; IV. Velasco, D. Aguilà, L. Barrios, I. Borilovic, O. Roubeau, J. Ribas-Ariño, M. Fumanal, S. Teat, G. Aromí, Chem. Sci. 2015, 6, 123-131; mJ. Overgaard, M. P. Waller, R. Piltz, J. A. Platts, P. Emseis, P. Leverett, P. A. Williams, D. E. Hibbs, J. Phys. Chem. A 2007, 111, 10123-10133; nW. Jauch, M. Reehuis, Phys. Rev. B. 2004, 70, 195121; oN. Ghadimi, C. B. Perry, P. P. Govender, H. M. Marques, Inorg. Chim. Acta 2016, 450, 269-278; pY.-Z. Zheng, W. Xue, M.-L. Tong, X.-M. Chen, S.-L. Zheng, Inorg. Chem. 2008, 47, 11202-11211; qD. Rutkowska-Zbik, M. Witko, G. Stochel, Theor. Chem. Acc. 2008, 120, 411-419.

[32] aF. Hueso-Ureña, S. B. Jiménez-Pulido, M. P. Fernández-Liencres, M. Fernández-Gómez, M. N. Moreno-Carretero, Dalton Trans. 2008, 6461-6466; bJ. Poater, M. Sola, A. Rimola, L. RodriguezSantiago, M. Sodupe, J. Phys. Chem. A 2004, 108, 6072-6078; cJ. Overgaard, I. Turel, D. E. Hibbs, Dalton Trans. 2007, 2171-2178; dN. Bouhmaida, M. A. Méndez-Rojas, A. n. Pérez-Benítez, G. Merino, B. Fraisse, N. E. Ghermani, Inorg. Chem. 2010, 49, 6443-6452; eW. Jauch, M. Reehuis, Acta Cryst. B. 2014, 70, 983-988; fF. Bertolotti, A. Forni, G. Gervasio, D. Marabello, E. Diana, Polyhedron 2012, 42, 118-127; gZ. Yousefi, H. Eshtiagh-Hosseini, A. Salimi, J. Soleimannejad, Acta Cryst. C 2015, 71, 386393; hA. Baryshnikova, B. Minaev, G. Baryshnikov, W.-H. Sun, Russ. J. Inorg. Chem. 2016, 61, 588593; iH.-Y. Zhao, F.-Y. Bai, Y.-H. Xing, Z.-P. Li, Y.-Z. Cao, X.-Q. Zeng, M.-F. Ge, J. Coord. Chem. 2010, 63, 435-447; jG. Zhang, X. Zhao, D. Chen, J. Phys. Chem. A 2013, 117, 10944-10950; kS. Pillet, M. Souhassou, C. Lecomte, P. Rabu, M. Drillon, C. Massobrio, Phys. Rev. B. 2006, 73, 115116 ; IY. V. Nelyubina, A. A. Korlyukov, I. V. Fedyanin, K. A. Lyssenko, Inorg. Chem. 2013, 52, 14355-14363; mH. H. Monfared, M. Vahedpour, M. M. Yeganeh, M. Ghorbanloo, P. Mayer, C. Janiak, Dalton Trans. 2011, 40, 1286-1294; nT. Lippmann, P. Blaha, N. H. Andersen, H. F. Poulsen, T. Wolf, J. R. Schneider, K.-H. Schwarz, Acta Crystallogr. Sect. A: Found. Crystallogr. 2003, 59, 437-451; oT. Lippmann, J. R. Schneider, Acta Crystallogr. Sect. A: Found. Crystallogr. 2000, 56, 575-584; pM. A. A. Azzellini, M. P. Abbott, A. Machado, M. T. M. Miranda, L. C. Garcia, G. F. Caramori, M. B. Gonçalves, H. M. Petrilli, A. Ferreira, J. Brazilian Chem. Soc. 2010, 21, 1303-1317; qM. Ghambarian, M. Ghashghaee, Z. Azizi, Physical Chemistry Research 2017, 5, 135-152; rR. Golbedaghi, S. Azimi, A. Molaei, M. Hatami, B. Notash, J. Mol. Struct. 2017; sF. Zapata, A. Caballero, A. Espinosa, A. Tárraga, P. Molina, Dalton Trans. 2010, 39, 5429-5431; tL. J. Farrugia, D. S. Middlemiss, R. Sillanpää, P. Seppälä, J. Phys. Chem. A 2008, 112, 9050-9067; uA. Fonari, E. S. Leonova, M. Y. Antipin, Polyhedron 2011, 30, 17101717; vM. Gryl, Acta Cryst. B. 2015, 71, 392-405; wA. Pakiari, Z. Jamshidi, J. Phys. Chem. A 2008, 112, 7969-7975; xR. Kakkar, R. Grover, P. Gahlot, Polyhedron 2006, 25, 759-766; yF. Turias, M. Solà, L. Falivene, L. Cavallo, A. Poater, Struct. Chem. 2016, 1, 409-417; zN. Lu, Y. Bu, Can. J. Chem. 2017; aaJ. S. Woertink, P. J. Smeets, M. H. Groothaert, M. A. Vance, B. F. Sels, R. A. Schoonheydt, E. I. Solomon, PNAS 2009, 106, 18908-18913; abS. Ghosh, S. I. Gorelsky, S. DeBeer George, J. M. Chan, I. Cabrito, D. M. Dooley, J. J. Moura, I. Moura, E. I. Solomon, J. Am. Chem. Soc. 2007, 129, 3955-3965; acY. I. Bolgova, E. Belogolova, V. Belyaeva, E. Timofeeva, O. Trofimova, Russ. Chem. Bull. 2017, 4, 1039-1046; adS. L. Birtles, PhD thesis, Witwatersrand University, Johannesburg, South Africa 2011; aeI. Bytheway, B. N. Figgis, A. N. Sobolev, Dalton Trans. 2001, 3285-3294; afM. M. Heravi, T. Hosseinnejad, N. Nazari, Can. J. Chem. 2017, 95, 530-536; agA. Naeimi, S. Saeednia, M. Yoosefian, H. A. Rudbari, V. M. Nardo, J. Chem. Sci 2015, 126, 1321-1328; ahY. Zhang, E. Oldfield, J. Am. Chem. Soc. 2008, 130, 3814-3823; aiC.-H. Guo, H.-S. Wu, X.-M. Zhang, J.-Y. Song, X. Zhang, J. Phys. Chem. $A$ 2009, 113, 6710-6723; ajA. Poater, J. Phys. Chem. A 2009, 113, 9030-9040; akJ. Kožíšek, N. K. Hansen, H. Fuess, Acta Cryst. B. 2002, 58, 463-470; alL. Kucková, L. Bučinský, J. Kožíšek, J. Mol. Struct. 2017, 1135, 186-196; amY. Yu, W. Shen, J. Zhang, R. He, M. Li, J. Mol. Model. 2008, 14, 237247.

[33] aM. Castillo, E. Romano, H. Lanús, S. Díaz, A. B. Altabef, S. Brandán, J. Mol. Struct. 2011, 994, 202208; bM. B. Pinto, A. L. Soares, A. Mella Orellana, H. A. Duarte, H. A. De Abreu, J. Phys. Chem. A 2017, 121, 2399-2409; cX. L. Fernández, PhD thesis, Universitat Rovira i Virgili 2004; dJ. R. 
Sambrano, L. Gracia, J. Andrés, S. Berski, A. Beltrán, The Journal of Physical Chemistry A 2004, 108, 10850-10860; eE. Drozdz, A. Kolezynski, RSC Advances 2017, 7, 28898-28908.

[34] aY. Ha, A. L. Tenderholt, R. H. Holm, B. Hedman, K. O. Hodgson, E. I. Solomon, J. Am. Chem. Soc. 2014, 136, 9094-9105; bD. Zhou, D. Ma, Y. Wang, X. Liu, X. Bao, Chem. Phys. Lett. 2003, 373, 46-51; cN. Fritsch, PhD thesis, Friedrich-Alexander-Universität Erlangen-Nürnberg 2014; dT.-a. Okamura, Y. Ushijima, Y. Omi, K. Onitsuka, Inorg. Chem. 2012, 52, 381-394; eJ. F. Van der Maelen, J. A. Cabeza, Inorg. Chem. 2012, 51, 7384-7391; fD. W. Brogden, Y. Turov, M. Nippe, G. Li Manni, E. A. Hillard, R. Clérac, L. Gagliardi, J. F. Berry, Inorg. Chem. 2014, 53, 4777-4790; gB. Griffe, G. Agrifoglio, J. L. Brito, F. Ruette, Catal. Today 2005, 107-108, 388-396; hJ. Hein, PhD thesis, Technische Universität München 2015; iS. Berski, F. R. Sensato, V. Polo, J. Andres, V. Safont, J. Phys. Chem. A 2010, 115, 514-522; jA. Sierraalta, Chem. Phys. Lett. 1994, 227, 557-560; kP. Macchi, A. Schultz, F. Larsen, B. Iversen, J. Phys. Chem. A 2001, 105, 9231-9242; IP. Abramov, A. Y. Laricheva, E. Peresypkina, I. Mirzaeva, N. Moroz, M. Sokolov, Inorg. Chim. Acta 2012, 383, 7-12; mA. J. Bridgeman, G. Cavigliasso, Dalton Trans. 2001, 3556-3563; nM. M. Cosper, F. Neese, A. V. Astashkin, M. D. Carducci, A. M. Raitsimring, J. H. Enemark, Inorg. Chem. 2005, 44, 1290-1301; oT. A. Bazhenova, K. A. Lyssenko, D. A. Kuznetsov, N. V. Kovaleva, Y. V. Manakin, T. A. Savinykh, A. F. Shestakov, Polyhedron 2014, 76, 108-116.

[35] aF. Miradji, F. Virot, S. Souvi, L. Cantrel, F. Louis, V. Vallet, J. Phys. Chem. A 2016, 120, 606-614; bL. Bučinský, G. E. Büchel, R. Ponec, P. Rapta, M. Breza, J. Kožíšek, M. Gall, S. Biskupič, M. Fronc, K. Schiessl, Eur. J. Inorg. Chem. 2013, 2013, 2505-2519; cA. A. Adeniyi, P. A. Ajibade, J. Mol. Model. 2013, 19, 1325-1338; dM. R. Sundberg, R. Ponec, Inorg. Chim. Acta 2006, 359, 899-906; eT. Zábojníková, R. Cajzl, J. Kljun, Z. Chval, I. Turel, J. V. Burda, J. Comput. Chem. 2016, 37, 17661780.

[36] aA. Neuhaus, A. Veldkamp, G. Frenking, Inorg. Chem. 1994, 33, 5278-5286; bU. Pidun, G. Frenking, Organometallics 1995, 14, 5325-5336; cG. M. Chiarella, F. A. Cotton, C. A. Murillo, Q. Zhao, Inorg. Chem. 2014, 53, 2288-2295; dM. Bortoluzzi, C. Evangelisti, F. Marchetti, G. Pampaloni, F. Piccinelli, S. Zacchini, Dalton Trans. 2016, 45, 15342-15349.

[37] aL.-Z. Zhu, C.-Z. Wang, L. Mei, L. Wang, Y.-H. Liu, Z.-T. Zhu, Y.-L. Zhao, Z.-F. Chai, W.-Q. Shi, CrystEngComm 2015, 17, 3031-3040; bV. r. Vallet, U. Wahlgren, I. Grenthe, J. Phys. Chem. A 2012, 116, 12373-12380; cJ.-C. Tourneux, J.-C. Berthet, T. Cantat, P. Thuéry, N. Mézailles, M. Ephritikhine, J. Am. Chem. Soc. 2011, 133, 6162-6165; dS. O. Odoh, G. Schreckenbach, Inorg. Chem. 2012, 52, 245-257; eP. L. Arnold, G. M. Jones, S. O. Odoh, G. Schreckenbach, N. Magnani, J. B. Love, Nat. Chem. 2012, 4, 221-227; fF. Chen, C.-z. Wang, Z.-j. Li, J.-h. Lan, Y.-q. Ji, Z.-f. Chai, Inorg. Chem. 2015, 54, 3829-3834; gB. Teyar, L. Belkhiri, K. Costuas, A. Boucekkine, K. Meyer, Inorg. Chem. 2016, 55, 2870-2881; hA. J. Lewis, H. Yin, P. J. Carroll, E. J. Schelter, Dalton Trans. 2014, 43, 10844-10851; iP. L. Arnold, E. Hollis, G. S. Nichol, J. B. Love, J.-C. Griveau, R. Caciuffo, N. Magnani, L. Maron, L. Castro, A. Yahia, J. Am. Chem. Soc. 2013, 135, 3841-3854; jD. E. Smiles, G. Wu, P. Hrobárik, T. W. Hayton, J. Am. Chem. Soc. 2016, 138, 814-825; kG. A. Shamov, G. Schreckenbach, T. N. Vo, Chem. Eur. J. 2007, 13, 4932-4947; ID. E. Smiles, G. Wu, N. Kaltsoyannis, T. W. Hayton, Chem. Sci. 2015, 6, 3891-3899; mM. Šulka, L. Cantrel, V. Vallet, J. Phys. Chem. A 2014, 118, 10073-10080; nY. Qiu, H. Hu, G. Chen, J. Li, Sci. China Chem. 2014, 57, 426-434; oC.-L. Xiao, C.-Z. Wang, L. Mei, X.-R. Zhang, N. Wall, Y.-L. Zhao, Z.-F. Chai, W.-Q. Shi, Dalton Trans. 2015, 44, 14376-14387; pA. F. F. de Lucena, PhD thesis, Universidade de Lisboa 2015; qD. D. Schnaars, A. J. Gaunt, T. W. Hayton, M. B. Jones, I. Kirker, N. Kaltsoyannis, I. May, S. D. Reilly, B. L. Scott, G. Wu, Inorg. Chem. 2012, 51, 85578566; rG. M. Jones, PhD thesis, Edinburgh University 2013; sQ.-Y. Wu, F.-W. Zhai, Y. Liu, L.-Y. Yuan, Z.-F. Chai, W.-Q. Shi, Dalton Trans. 2016, 45, 14988-14997; tA. V. Vologzhanina, A. V. Savchenkov, A. O. Dmitrienko, A. A. Korlyukov, I. S. Bushmarinov, D. V. Pushkin, L. B. Serezhkina, J. Phys. Chem. A 2014, 118, 9745-9752; uV. V. Zhurov, E. A. Zhurova, A. I. Stash, A. A. Pinkerton, J. Phys. Chem. A 2011, 115, 13016-13023; vQ. Huang, PhD thesis, UCL (University College London) 2016; wB. Kosog, PhD thesis, Friedrich-Alexander-Universität Erlangen-Nürnberg 2012; xK.-Q. Hu, L.-Z. Zhu, C.-Z. Wang, L. Mei, Y.-H. Liu, Z.-Q. Gao, Z.-F. Chai, W.-Q. Shi, Cryst. Growth Des. 2016, 16, 4886-4896; yA. Prestianni, L. Joubert, A. Chagnes, G. Cote, C. Adamo, PCCP 2011, 13, 1937119377; zN. Jiang, W. E. Schwarz, J. Li, Inorg. Chem. 2015, 54, 7171-7180; aaD. Rios, M. del Carmen Michelini, A. F. Lucena, J. Marçalo, J. K. Gibson, J. Am. Chem. Soc. 2012, 134, 15488-15496; abP. Miró, J. Ling, J. Qiu, P. C. Burns, L. Gagliardi, C. J. Cramer, Inorg. Chem. 2012, 51, 8784-8790; acB. Zhang, X. Guo, S. Xie, X. Liu, C. Ling, H. Ma, M. Yu, J. Li, RSC Advances 2016, 6, 81995-82005; adX. Guo, X.-G. Xiong, C. Li, H. Gong, P. Huai, J. Hu, C. Jin, L. Huang, G. Wu, Inorg. Chim. Acta 2016, 441, 117-125; aeH. Xiao, H.-S. Hu, W. H. E. Schwarz, J. Li, J. Phys. Chem. A 2010, 114, 8837-8844.

[38] aI. Kirker, PhD thesis, UCL (University College London) 2013; bG. Gopakumar, B. Sreenivasulu, A. Suresh, C. Brahmmananda Rao, N. Sivaraman, M. Joseph, A. Anoop, J. Phys. Chem. A 2016, 120, 
4201-4210; cP. Li, W. Niu, T. Gao, RSC Advances 2017, 7, 4291-4296; dN. Kaltsoyannis, Dalton Trans. 2016, 45, 3158-3162.

[39] aC. W. Spicer, C. F. Lovitt, G. S. Girolami, Organometallics 2012, 31, 4894-4903; bM. Zhang, K. Cheng, H. Yan, Q. Wei, B. Zheng, Sci. Rep. 2016, 6; cD. J. Wolstenholme, K. T. Traboulsee, A. Decken, G. S. McGrady, Organometallics 2010, 29, 5769-5772; dJ. Zhu, E.-L. Zins, M. E. Alikhani, J. Mol. Model. 2016, 22, 294; eX. Wang, Y. Wang, S. Li, Y. Zhang, P. Ma, J. Phys. Chem. A 2016, 120, 5457-5463; fJ. R. De Lile, T. Heine, S. Zhou, Comput. Mater. Sci. 2017, 129, $24-36$.

[40] aA. C. Filippou, S. Schneider, G. Schnakenburg, Angew. Chem. Int. Ed. 2003, 42, 4486-4489; bC. J. Czerwinski, E. O. Fetisov, I. P. Gloriozov, Y. F. Oprunenko, Dalton Trans. 2013, 42, 10487-10494; cL.C. Wu, M. K. Thomsen, S. R. Madsen, M. Schmoekel, M. R. Jørgensen, M.-C. Cheng, S.-M. Peng, Y.S. Chen, J. Overgaard, B. B. Iversen, Inorg. Chem. 2014, 53, 12489-12498; dE. P. Beaumier, B. S. Billow, A. K. Singh, S. M. Biros, A. L. Odom, Chem. Sci. 2016, 7, 2532-2536; eM. Palusiak, J. Organomet. Chem. 2007, 692, 3866-3873; fJ. Liu, H. Liu, Y. Li, H. Wang, J. Biol. Phys. 2014, 40, 313323; gD. B. DuPré, J. Phys. Chem. A 2009, 113, 1559-1563.

[41] aG. Vanko, A. Bordage, M. Papai, K. Haldrup, P. Glatzel, A. M. March, G. Doumy, A. Britz, A. Galler, T. Assefa, J. Phys. Chem. C 2015, 119, 5888-5902; bM. P. Mehn, S. D. Brown, T. K. Paine, W. W. Brennessel, C. J. Cramer, J. C. Peters, L. Que Jr, Dalton Trans. 2006, 1347-1351; cS. A. Sulway, PhD thesis, Manchester University 2012; dD. Gallego, S. Inoue, B. Blom, M. Driess, Organometallics 2014, 33, 6885-6897; eM.-E. Moret, J. C. Peters, J. Am. Chem. Soc. 2011, 133, 18118-18121; fS. Mebs, B. Braun, R. Kositzki, C. Limberg, M. Haumann, Inorg. Chem. 2015, 54, 11606-11624; gK. L. Fillman, J. A. Przyojski, M. H. Al-Afyouni, Z. J. Tonzetich, M. L. Neidig, Chem. Sci. 2015, 6, 1178-1188; hS. Pillet, V. Legrand, H.-P. Weber, M. Souhassou, J.-F. Létard, P. Guionneau, C. Lecomte, Z. Kristallogr. 2008, 223, 235-249; iR. Ghiasi, M. Daghighi Asl, S. Azmayesh, P. Makkipour, IJNC 2014, 1, 85-96; jS. Ouanounou, W.-C. Shih, T.-G. Ong, G. P. Yap, I. Korobkov, S. Gorelsky, D. Richeson, Dalton Trans. 2016, 45, 14327-14334; kN. Sanina, G. Kozub, T. Kondrat'eva, G. Shilov, D. Korchagin, N. Emel'yanova, O. K. Poleshchuk, A. Chernyak, A. Kulikov, F. Mushenok, J. Mol. Struct. 2013, 1041, 183-189; IM. Ansari, N. Vyas, A. Ansari, G. Rajaraman, Dalton Trans. 2015, 44, 15232-15243; mK. Mierzwicki, S. Berski, Z. Latajka, Chem. Phys. Lett. 2011, 507, 29-36; nX. Sun, J. Du, J. Theor. Comput. Chem. 2017, 16, 1750036; oN. L. Fry, PhD thesis, University of California, Santa Cruz 2012; pY. Luo, Y. Li, H. Yu, J. Zhao, Y. Chen, Z. Hou, J. Qu, Organometallics 2011, 31, 335-344.

[42] aJ. A. Przyojski, M. L. Kiewit, K. L. Fillman, H. D. Arman, Z. J. Tonzetich, Inorg. Chem. 2015, 54, 9637-9645; bA. S. Eisenberg, I. V. Likhtina, V. S. Znamenskiy, R. L. Birke, J. Phys. Chem. A 2012, 116, 6851-6869; cT. Jurca, S. Ouanounou, W.-C. Shih, T.-G. Ong, G. P. A. Yap, I. Korobkov, S. Gorelsky, D. Richeson, Dalton Trans. 2016, 45, 14327-14334; dS. Cong, L. K. Yan, S. Z. Wen, W. Guan, Z. M. Su, Theor. Chem. Acc. 2011, 130, 1043-1053; eS. J. Bonyhady, J. M. Goldberg, N. Wedgwood, T. R. Dugan, A. G. Eklund, W. W. Brennessel, P. L. Holland, Inorg. Chem. 2015, 54, 51485150; fL. Zhang, Y. Liu, L. Deng, J. Am. Chem. Soc. 2014, 136, 15525-15528; gP. P. Govender, I. Navizet, C. B. Perry, H. M. Marques, J. Phys. Chem. A 2013, 117, 3057-3068; hY. Jing, X. Chen, X. Yang, Organometallics 2015, 34, 5716-5722; iS. Mebs, J. Henn, B. Dittrich, C. Paulmann, P. Luger, J. Phys. Chem. A 2009, 113, 8366-8378; jS. Mathura, D. Sannasy, A. S. de Sousa, C. B. Perry, I. Navizet, H. M. Marques, J. Inorg. Biochem. 2013, 123, 66-79; kT. Zhu, P. Ning, L. Tang, K. Li, S. Bao, X. Jin, X. Song, X. Zhang, S. Han, AIP Advances 2017, 7, 025012.

[43] aC. J. C. Richter, PhD thesis, Swiss Federal Institute of Technology (ETH), Zurich 2006; bR.-Y. Huang, H. Xu, S.-Y. Ye, G.-H. Wu, X.-Q. Zhao, Y. Wang, G.-X. Liu, J. Mol. Struct. 2013, 1036, 235243; cT. C. Davenport, PhD thesis, University of California, Berkeley 2012; dZ. Mahdavifar, N. Abbasi, Physica E 2014, 56, 268-276; eA. V. Vologzhanina, A. A. Korlyukov, V. V. Avdeeva, I. N. Polyakova, E. A. Malinina, N. T. Kuznetsov, J. Phys. Chem. A 2013, 117, 13138-13150; fA. V. Vologzhanina, S. V. Kats, L. V. Penkova, V. A. Pavlenko, N. N. Efimov, V. V. Minin, I. L. Eremenko, Acta Cryst. B. 2015, 71, 543-554; gF. Hueso-Urena, S. B. Jimenez-Pulido, M. P. Fernandez-Liencres, M. Fernandez-Gomez, M. N. Moreno-Carretero, Dalton Trans. 2008, 6461-6466; hA. Casitas, A. Poater, M. Sola, S. S. Stahl, M. Costas, X. Ribas, Dalton Trans. 2010, 39, 10458-10463; iA. Luna, B. Amekraz, J. Tortajada, Chem. Phys. Lett. 1997, 266, 31-37; jD. E. P. Fonseca, PhD thesis, Universidade Federal de Santa Catarina,Florianópolis 2013; kK. Kisowska, S. Berski, Z. Latajka, J. Comput. Chem. 2008, 29, 2677-2692; IL. Guo, W. Dong, S. Zhang, RSC advances 2014, 4, 41956-41967; mM. Wang, R.-Y. Huang, Z.-Q. Wang, G.-H. Wu, Inorg. Chem. Commun. 2015, 56, 41-44.

[44] aL. Yan, M. Jin, P. Song, Z. Su, J. Phys. Chem. B 2010, 114, 3754-3758; bJ. J. Curley, N. A. Piro, C. C. Cummins, Inorg. Chem. 2009, 48, 9599-9601; cG. H. Timmer, J. F. Berry, Chem. Sci. 2012, 3, 30383052; dA. Poater, X. Solans-Monfort, E. Clot, C. Copéret, O. Eisenstein, Dalton Trans. 2006, 30773087; eA. Eizawa, K. Arashiba, H. Tanaka, S. Kuriyama, Y. Matsuo, K. Nakajima, K. Yoshizawa, Y. Nishibayashi, Nat. Commun. 2017, 8, 14874; fG. C. Fortman, PhD thesis, Miami University 2009; gT. Wagener, G. Frenking, Inorg. Chem. 1998, 37, 1805-1811. 
[45] aP. Spuhler, M. Lein, G. Frenking, Z. Anorg. Allg. Chem. 2003, 629, 803-815; bS. Baskaran, P. Balu, C. Sivasankar, J. Chem. Sci. 2015, 127, 83-94; cR. Ghiasi, M. Nemati, A. H. Hakimioun, J. Chil. Chem. Soc. 2016, 61, 2921-2928; dJ. Song, M. B. Hall, J. Am. Chem. Soc. 1993, 115, 327-336.

[46] aT. W. Hayton, Chem. Commun. 2013, 49, 2956-2973; bA. R. Fox, C. C. Cummins, J. Am. Chem. Soc. 2009, 131, 5716-5717; cB. Vlaisavljevich, L. Gagliardi, X. Wang, B. Liang, L. Andrews, I. Infante, Inorg. Chem. 2010, 49, 9230-9235; dD. M. King, F. Tuna, E. J. McInnes, J. McMaster, W. Lewis, A. J. Blake, S. T. Liddle, Nat. Chem. 2013, 5, 482-488; eB. M. Gardner, S. T. Liddle, Eur. J. Inorg. Chem. 2013, 2013, 3753-3770; fQ.-Y. Wu, C.-Z. Wang, J.-H. Lan, C.-L. Xiao, X.-K. Wang, Y.-L. Zhao, Z.-F. Chai, W.-Q. Shi, Inorg. Chem. 2014, 53, 9607-9614; gA. Hervé, Y. Bouzidi, J.-C. Berthet, L. Belkhiri, P. Thuéry, A. Boucekkine, M. Ephritikhine, Inorg. Chem. 2014, 53, 6995-7013; hA. Hervé, Y. Bouzidi, J.-C. Berthet, L. Belkhiri, P. Thuéry, A. Boucekkine, M. Ephritikhine, Inorg. Chem. 2015, 54, 24742490; iK. K. Pandey, Inorg. Chem. Commun. 2013, 37, 4-6; jB. Vlaisavljevich, L. Andrews, X. Wang, Y. Gong, G. P. Kushto, B. E. Bursten, J. Am. Chem. Soc. 2016, 138, 893-905; kM. Gregson, E. Lu, D. P. Mills, F. Tuna, E. J. L. McInnes, C. Hennig, A. C. Scheinost, J. McMaster, W. Lewis, A. J. Blake, A. Kerridge, S. T. Liddle, Nat. Commun. 2017, 8, 14137; IB. M. Gardner, S. T. Liddle, Chem. Commun. 2015, 51, 10589-10607; mA. Elkechai, F. Kias, F. Talbi, A. Boucekkine, J. Mol. Model. 2014, 20, 2294; nP. Di Pietro, A. Kerridge, Inorg. Chem. 2015, 55, 573-583; oP. Di Pietro, A. Kerridge, PCCP 2016, 18, 16830-16839; pP. Di Pietro, A. Kerridge, PCCP 2017, 19, 7546-7559; qZ. Huang, L. Sun, Y. Yuan, Y. Li, X. Wang, Inorg. Chem. 2016; rN. Qu, D.-M. Su, Q.-Y. Wu, W.-Q. Shi, Q.-J. Pan, Comput. Theor. Chem. 2017, 1108, 29-39; sD. M. King, J. McMaster, F. Tuna, E. J. McInnes, W. Lewis, A. J. Blake, S. T. Liddle, J. Am. Chem. Soc. 2014, 136, 5619-5622; tX. Guo, L. Huang, C. Li, J. Hu, G. Wu, P. Huai, PCCP 2015, 17, 14662-14673.

[47] aD. Tiana, E. Francisco, M. Blanco, P. Macchi, A. Sironi, A. Martín Pendás, J. Chem. Theory Comput. 2010, 6, 1064-1074; bC. L. Firme, D. d. L. Pontes, O. A. Antunes, Chem. Phys. Lett. 2010, 499, 193-198; cO. J. Cooper, D. P. Mills, J. McMaster, F. Tuna, E. McInnes, W. Lewis, A. J. Blake, S. T. Liddle, Chem. Eur. J. 2013, 19, 7071-7083; dB. C. Bailey, H. Fan, J. C. Huffman, M.-H. Baik, D. J. Mindiola, J. Am. Chem. Soc. 2007, 129, 8781-8793; eM. Kamitani, B. Pinter, K. Searles, M. G. Crestani, A. Hickey, B. C. Manor, P. J. Carroll, D. J. Mindiola, J. Am. Chem. Soc. 2015, 137, 11872-11875; fJ. G. Andino, H. Fan, A. R. Fout, B. C. Bailey, M.-H. Baik, D. J. Mindiola, J. Organomet. Chem. 2011, 696, 4138-4146; gM. Lein, J. A. Harrison, A. J. Nielson, Dalton Trans. 2013, 42, 10939-10951; hR. F. Bader, C. F. Matta, Inorg. Chem. 2001, 40, 5603-5611; iH.-F. Li, X.-Y. Kuang, H.-Q. Wang, Dalton Trans. 2011, 40, 4578-4589; jJ. Pilme, B. Silvi, M. E. Alikhani, J. Phys. Chem. A 2003, 107, 4506-4514; kS. Roy, U. Rosenthal, E. D. Jemmis, Acc. Chem. Res. 2014, 47, 2917-2930; IG. V. Oshovsky, B. Hessen, J. N. Reek, B. d. Bruin, Organometallics 2011, 30, 6067-6070; mI. Vidal, S. Melchor, J. A. Dobado, J. Phys. Chem. A 2008, 112, 3414-3423.

[48] aG. Frenking, M. Solà, S. F. Vyboishchikov, J. Organomet. Chem. 2005, 690, 6178-6204; bB. S. Billow, R. D. Bemowski, S. A. DiFranco, R. J. Staples, A. L. Odom, Organometallics 2015, 34, 4567-4573; cC. Makanjee, Master's thesis, Rhodes University 2012; dP. P. Samuel, R. Neufeld, K. C. Mondal, H. W. Roesky, R. Herbst-Irmer, D. Stalke, S. Demeshko, F. Meyer, V. C. Rojisha, S. De, Chem. Sci. 2015, 6, 3148-3153; eC.-C. Wang, Y. Wang, H.-J. Liu, K.-J. Lin, L.-K. Chou, K.-S. Chan, J. Phys. Chem. A 1997, 101, 8887-8901; fH. Li, H. Feng, W. Sun, Y. Xie, R. B. King, H. F. Schaefer III, New J. Chem. 2011, 35, 1718-1729; gB. Boucher, J.-F. Halet, M. Kohout, Comput. Theor. Chem. 2015, 1068, 134-141; hR. Ghiasi, J. Theor. Comput. Chem. 2015, 14, 1550022; iI. Fernández, M. J. Mancheño, R. Vicente, L. A. López, M. A. Sierra, Chem. Eur. J. 2008, 14, 11222-11230; jU. Flierler, PhD thesis, Niedersächsische Staats-und Universitätsbibliothek Göttingen 2009.

[49] aC. Gatti, D. Lasi, Faraday Discuss. 2007, 135, 55-78; bK. Götz, M. Kaupp, H. Braunschweig, D. Stalke, Chem. Eur. J. 2009, 15, 623-632; cJ. F. Van der Maelen, J. A. Cabeza, Theor. Chem. Acc. 2016, 135, 1-11; dX. Yan, X. Li, Z. Sun, Q. Li, L. Meng, New J. Chem. 2016, 40, 1988-1996; eJ. Brugos, J. A. Cabeza, P. Garcia-Alvarez, E. Perez-Carreno, J. F. Van der Maelen, Dalton Trans. 2017, 46, 40094017; fD. A. Valyaev, R. Brousses, N. Lugan, I. Fernández, M. A. Sierra, Chem. Eur. J. 2011, 17, 66026605; gY. Ortin, N. Lugan, S. Pillet, M. Souhassou, C. Lecomte, K. Costuas, J.-Y. Saillard, Inorg. Chem. 2005, 44, 9607-9609.

[50] aY. Chen, PhD thesis, Philipps-Universität Marburg 2000; bT. R. Kégl, L. Kollár, T. Kégl, J. Quantum Chem. 2014, 2014; cJ. Uddin, C. Boehme, G. Frenking, Organometallics 2000, 19, 571-582; dP. Parameswaran, G. Frenking, Chem. Eur. J. 2009, 15, 8807-8816; eA. I. Konovalov, PhD thesis, Institut Català d'Investigació Química (ICIQ) 2015; fA. Rossin, PhD thesis, Cardiff University 2004; gB. Rohrmoser, G. Eickerling, M. Presnitz, W. Scherer, V. Eyert, R.-D. Hoffmann, U. C. Rodewald, C. Vogt, R. Pöttgen, J. Am. Chem. Soc. 2007, 129, 9356-9365; hS. A. Decker, PhD thesis, University of Alberta 2000; iM. A. Alvarez, M. E. García, D. García-Vivó, A. Ramos, M. A. Ruiz, Inorg. Chem. 2012, 51, 3698-3706; jH. Chevreau, C. Martinsky, A. Sevin, C. Minot, B. Silvi, New J. Chem. 2003, 27, 1049-1053; kL. J. Farrugia, C. Evans, M. Tegel, J. Phys. Chem. A 2006, 110, 7952-7961; IP. Macchi, L. 
Garlaschelli, A. Sironi, J. Am. Chem. Soc. 2002, 124, 14173-14184; mH. Braunschweig, Q. Ye, A. Vargas, K. Radacki, A. Damme, Angew. Chem. Int. Ed. 2013, 52, 10657-10660; nE. P. Malar, Theor. Chem. Acc. 2005, 114, 213-221.

[51] aJ. Overgaard, J. A. Platts, B. B. Iversen, Acta Cryst. B. 2009, 65, 715-723; bL. J. Farrugia, C. Evans, C.R. Chim. 2005, 8, 1566-1583; cC. B. Perry, N. Shin, M. A. Fernandes, H. M. Marques, Dalton Trans. 2013, 42, 7555-7561; dT. Kégl, F. Ungváry, J. Organomet. Chem. 2007, 692, 1825-1833; eS. P. George, PhD thesis, California University, San Diego 2014.

[52] aT. Kégl, R. Ponec, L. Kollár, J. Phys. Chem. A 2011, 115, 12463-12473; bR. Ghiasi, A. H. Hakimyoon, J. Mex. Chem. Soc. 2012, 56, 100-104; cG. Eickerling, R. Mastalerz, V. Herz, W. Scherer, H.-J. Himmel, M. Reiher, J. Chem. Theory Comput. 2007, 3, 2182-2197; dF. Koch, H. Schubert, P. Sirsch, A. Berkefeld, Dalton Trans. 2015, 44, 13315-13324; eW. Scherer, G. Eickerling, D. Shorokhov, E. Gullo, G. S. McGrady, P. Sirsch, New J. Chem. 2006, 30, 309-312; fJ. Du, X. Sun, J. Chen, G. Jiang, RSC Advances 2014, 4, 44786-44794; gR. Ghiasi, S. Hashemian, O. Irajee, J. Korean Chem. Soc. 2011, 55; hW. Scherer, C. Hauf, M. Presnitz, E. W. Scheidt, G. Eickerling, V. Eyert, R. D. Hoffmann, U. C. Rodewald, A. Hammerschmidt, C. Vogt, Angew. Chem. Int. Ed. 2010, 49, 1578-1582; iW. H. Harman, T. P. Lin, J. C. Peters, Angew. Chem. Int. Ed. 2014, 53, 1081-1086; jP. Macchi, D. M. Proserpio, A. Sironi, J. Am. Chem. Soc. 1998, 120, 1447-1455; kN. Hebben, H. J. Himmel, G. Eickerling, C. Herrmann, M. Reiher, V. Herz, M. Presnitz, W. Scherer, Chem. Eur. J. 2007, 13, 10078-10087; IL. S. Jongbloed, PhD thesis, Amsterdam University 2016.

[53] aC. Boehme, G. Frenking, Organometallics 1998, 17, 5801-5809; bM. Boehme, G. Frenking, M. T. Reetz, Organometallics 1994, 13, 4237-4245; cP. Balu, V. Kannappan, R. Kumar, Advances in Chemistry 2016, 2016; dA. Poater, M. Duran, P. Jaque, A. Toro-Labbe, M. Sola, J. Phys. Chem. B 2006, 110, 6526-6536; eC. M. Wyss, PhD thesis, Georgia Institute of Technology 2015; fG. Wu, Y.-W. Li, H.-W. Xiang, Y.-Y. Xu, Y.-H. Sun, H. Jiao, J. Mol. Struct. THEOCHEM 2003, 637, 101-107; gC. Calle, A. Schweiger, G. Mitrikas, Inorg. Chem. 2007, 46, 1847-1855; hQ. Meng, M. Li, J. Mol. Struct. THEOCHEM 2006, 765, 13-20; iM. E. Alikhani, L. Manceron, J. Mol. Spectrosc. 2015, 310, 32-38; jZ. Huang, Y. Yuan, L. Sun, X. Wang, Y. Li, RSC Advances 2016, 6, 84016-84024; kG. Sánchez-Sanz, I. Alkorta, J. Elguero, M. Yáñez, O. Mó, PCCP 2012, 14, 11468-11477; IK. Freitag, H. Banh, C. Gemel, R. W. Seidel, S. Kahlal, J.-Y. Saillard, R. A. Fischer, Chem. Commun. 2014, 50, 8681-8684; mC. Geng, J. Li, T. Weiske, M. Schlangen, S. Shaik, H. Schwarz, J. Am. Chem. Soc. 2017, 139, 1684-1689; nJ. R. Shakirova, E. V. Grachova, V. V. Gurzhiy, I. O. Koshevoy, A. S. Melnikov, O. V. Sizova, S. P. Tunik, A. Laguna, Dalton Trans. 2012, 41, 2941-2949; oX.-B. Wang, Y.-L. Wang, J. Yang, X.-P. Xing, J. Li, L.-S. Wang, J. Am. Chem. Soc. 2009, 131, 16368-16370; pP. I. Dem 'yanov, R. M. Gschwind, Organometallics 2006, 25, 5709-5723; qK. Lammertsma, A. W. Ehlers, M. L. McKee, J. Am. Chem. Soc. 2003, 125, 14750-14759; rY. Liu, Z. Luo, J. Z. Zhang, F. Xia, J. Phys. Chem. A 2016, 120, 64856492; sA. C. Tsipis, D. N. Gkarmpounis, J. Comput. Chem. 2012, 33, 2318-2331; tA. C. Tsipis, Organometallics 2012, 31, 7206-7212; uN. Grzegorzek, M. Pawlicki, L. Szterenberg, L. LatosGrażyński, J. Am. Chem. Soc. 2009, 131, 7224-7225; vU. Jayarathne, T. J. Mazzacano, S. Bagherzadeh, N. P. Mankad, Organometallics 2013, 32, 3986-3992; wN. Grzegorzek, E. b. Nojman, L. Szterenberg, L. Latos-Grażyński, Inorg. Chem. 2013, 52, 2599-2606; xR. Grande-Aztatzi, J. M. Mercero, E. Matito, G. Frenking, J. M. Ugalde, PCCP 2017, 19, 9669-9675; yG. Huang, Y. Xia, ACS Catalysis 2015, 5, 859-868.

[54] aX. Liu, D. R. Salahub, Can. J. Chem. 2015, 94, 282-292; bH.-S. Hu, Y.-H. Qiu, X.-G. Xiong, W. H. E. Schwarz, J. Li, Chem. Sci. 2012, 3, 2786-2796; cM. E. García, D. García-Vivó, M. A. Ruiz, S. Alvarez, G. Aullón, Organometallics 2007, 26, 5912-5921; dN. Curado, M. Carrasco, E. Álvarez, C. Maya, R. Peloso, A. Rodríguez, J. López-Serrano, E. Carmona, J. Am. Chem. Soc. 2015, 137, 12378-12387; eT. R. Cundari, M. S. Gordon, Organometallics 1992, 11, 55-63; fR. Stegmann, A. Neuhaus, G. Frenking, J. Am. Chem. Soc. 1993, 115, 11930-11938; gM. Carrasco, M. Faust, R. Peloso, A. Rodríguez, J. LópezSerrano, E. Álvarez, C. Maya, P. P. Power, E. Carmona, Chem. Commun. 2012, 48, 3954-3956; hT. A. N. Nguyen, T. P. L. Huynh, V. T. Pham, J. Vietnam. Environ. 2014, 6, 142-148; iR. Ramalakshmi, K. Maheswari, D. Sharmila, A. Paul, T. Roisnel, J.-F. Halet, S. Ghosh, Dalton Trans. 2016, 45, 1631716324; jV. Caliman, P. B. Hitchcock, J. F. Nixon, L. Nyulaszi, N. Sakarya, Chem. Commun. 1997, 1305-1306; kM. a. Alonso, M. A. Alvarez, M. E. García, D. García-Vivó, M. A. Ruiz, Inorg. Chem. 2010, 49, 8962-8976; IK. K. Pandey, M. Lein, G. Frenking, J. Am. Chem. Soc. 2003, 125, 1660-1668; mM. Ray, PhD thesis, Kyoto University 2009; nM. Alvarez, M. E. García, D. García-Vivó, S. Menéndez, M. A. Ruiz, Chem. Eur. J. 2016, 22, 8763-8767; oM. A. Alvarez, M. Casado-Ruano, M. E. García, D. García-Vivó, M. A. Ruiz, Inorg. Chem. 2017, 56, 11336-11351.

[55] aJ. A. Cabeza, J. F. Van der Maelen, S. García-Granda, Organometallics 2009, 28, 3666-3672; bJ. Malecki, A. Maroń, M. Serda, M. Dolezal, J. Polański, Polyhedron 2012, 41, 104-114; cA. May, N. Ouddai, J. Struct. Chem. 2012, 53, 220-227; dR. Ghiasi, M. Manochehri, N. Yadegari, Russ. J. Phys. Chem. A 2013, 87, 1506-1514; eG. Occhipinti, H.-R. Bjørsvik, V. R. Jensen, J. Am. Chem. Soc. 2006, 
128, 6952-6964; fP. R. Remya, C. H. Suresh, Dalton Trans. 2015, 44, 17660-17672; gA. O. Borissova, M. Y. Antipin, D. S. Perekalin, K. A. Lyssenko, CrystEngComm 2008, 10, 827-832; hG. Gervasio, R. Bianchi, D. Marabello, Chem. Phys. Lett. 2005, 407, 18-22.

[56] aS. F. Vyboishchikov, G. Frenking, Chem. Eur. J. 1998, 4, 1428-1438; bR. Stegmann, G. Frenking, Organometallics 1995, 14, 5308-5315; cP. R. Remya, C. H. Suresh, Dalton Trans. 2016, 45, 1769-1778; dS. F. Vyboishchikov, G. Frenking, Chem. Eur. J. 1998, 4, 1439-1448; eE. D. Jemmis, K. T. Giju, Organometallics 1997, 16, 1425-1429; fG. Frenking, U. Pidun, Dalton Trans. 1997, 1653-1662; gM. A. Alvarez, M. E. García, D. García-Vivó, M. A. Ruiz, M. F. Vega, Dalton Trans. 2014, 43, 16044-16055; hM. A. Alvarez, M. E. García, D. García-Vivó, M. A. Ruiz, M. F. Vega, Organometallics 2010, 29, $512-$ 515; iX. Sun, J. Du, Eur. Phys. J. D 2014, 68, 211; jT. Chen, X.-H. Zhang, C. Wang, S. Chen, Z. Wu, L. Li, K. R. Sorasaenee, J. B. Diminnie, H. Pan, I. A. Guzei, Organometallics 2005, 24, 1214-1224; kA. C. Filippou, A. I. Philippopoulos, G. Schnakenburg, Organometallics 2003, 22, 3339-3341.

[57] aL. Petit, L. Joubert, P. Maldivi, C. Adamo, J. Am. Chem. Soc. 2006, 128, 2190-2191; bJ. T. Lyon, H.S. Hu, L. Andrews, J. Li, PNAS 2007, 104, 18919-18924; cZ. R. Turner, PhD thesis, Edinburgh University 2011; dC.-Z. Wang, J. K. Gibson, J.-H. Lan, Q.-Y. Wu, Y.-L. Zhao, J. Li, Z.-F. Chai, W.-Q. Shi, Dalton Trans. 2015, 44, 17045-17053; eE. Di Santo, M. d. C. Michelini, N. Russo, Organometallics 2009, 28, 3716-3726; fI. Kirker, N. Kaltsoyannis, Dalton Trans. 2011, 40, 124-131; gP. L. Arnold, Z. R. Turner, N. Kaltsoyannis, P. Pelekanaki, R. M. Bellabarba, R. P. Tooze, Chem. Eur. J. 2010, 16, 96239629; hA. H. Greif, P. Hrobárik, J. Autschbach, M. Kaupp, PCCP 2016, 18, 30462-30474; iM. F. Zalazar, V. M. Rayón, A. Largo, J. Chem. Phys. 2013, 138, 114307.

[58] aA. Oppermann, R. Dick, C. Wehrhahn, U. Flörke, S. Herres-Pawlis, G. Henkel, Eur. J. Inorg. Chem. 2016, 2016, 3744-3755; bS. A. Sadjadi, PhD thesis, Hong Kong University 2013; cS. Dinda, A. G. Samuelson, Chem. Eur. J. 2012, 18, 3032-3042; dP. I. Dem'yanov, P. M. Polestshuk, V. V. Kostin, Chem. Eur. J. 2017, 23, 3257-3261; eX. Li, J. Cai, Mol. Phys. 2017, 115, 1544-1554.

[59] aH. F. Dos Santos, D. Paschoal, J. V. Burda, Chem. Phys. Lett. 2012, 548, 64-70; bX. Li, J. Comput. Chem. 2014, 35, 923-931; cP. Jerabek, PhD thesis, Philipps-Universität Marburg 2015; dT. B. Anisimova, M. A. Kinzhalov, M. F. C. G. da Silva, A. S. Novikov, V. Y. Kukushkin, A. J. Pombeiro, K. V. Luzyanin, New J. Chem. 2017, 41, 3246-3250; eW.-L. Li, Y. Li, C.-Q. Xu, X.-B. Wang, E. Vorpagel, J. Li, Inorg. Chem. 2015, 54, 11157-11167; fH.-T. Liu, X.-G. Xiong, P. D. Dau, Y.-L. Wang, J. Li, L.-S. Wang, Chem. Sci. 2011, 2, 2101-2108.

[60] aY. Sunada, S. Ishida, F. Hirakawa, Y. Shiota, K. Yoshizawa, S. Kanegawa, O. Sato, H. Nagashima, T. Iwamoto, Chem. Sci. 2016, 7, 191-198; bJ. L. Kneebone, V. E. Fleischauer, S. L. Daifuku, A. A. Shaps, J. M. Bailey, T. E. Iannuzzi, M. L. Neidig, Inorg. Chem. 2015, 55, 272-282; cB. Stadelmann, J. Bender, D. Förster, W. Frey, M. Nieger, D. Gudat, Dalton Trans. 2015, 44, 6023-6031; dS. Cenedese, PhD thesis, Milan University 2011.

[61] aM. Feliz, R. Llusar, J. Andrés, S. Berski, B. Silvi, New J. Chem. 2002, 26, 844-850; bM. A. Alvarez, M. E. García, D. García-Vivó, R. Lozano, A. Ramos, M. A. Ruiz, Inorg. Chem. 2014, 53, 11261-11273; cM. Fleischmann, PhD thesis, Universität Regensburg 2016.

[62] aG. Gibbs, R. T. Downs, C. Prewitt, K. M. Rosso, N. Ross, D. F. Cox, J. Phys. Chem. B 2005, 109, 21788-21795; bS. A. Vedha, R. V. Solomon, P. Venuvanalingam, PCCP 2014, 16, 10698-10707; cY. Aray, D. Vega, J. Rodriguez, A. B. Vidal, M. E. Grillo, S. Coll, J. Phys. Chem. B 2009, 113, 3058-3070; dD. C. Onwudiwe, M. M. Kabanda, E. E. Ebenso, E. Hosten, J. Chem. Sci. 2016, 128, 1081-1093.

[63] aA. Morales-García, A. L. Soares Jr, E. C. Dos Santos, H. A. de Abreu, H. I. A. Duarte, J. Phys. Chem. $A$ 2014, 118, 5823-5831; bR. Ghiasi, J. Sulfur. Chem. 2012, 33, 93-100; cK. M. Rosso, M. Hochella, Surf. Sci. 1999, 423, 364-374; dX. Zhang, X. Li, Y. Zeng, S. Zheng, L. Meng, Dalton Trans. 2015, 44, 1283-1291; eJ. Andrés, S. Berski, M. Feliz, R. Llusar, F. Sensato, B. Silvi, C.R. Chim. 2005, 8, 14001412; fB. Ni, J. R. Kramer, N. H. Werstiuk, J. Phys. Chem. A 2003, 107, 2890-2897; gA. Pakiari, Z. Jamshidi, J. Phys. Chem. A 2010, 114, 9212-9221.

[64] aT. Li, PhD thesis, The Ohio State University 2016; bS. I. Gorelsky, J. Chem. Theory Comput. 2012, 8, 908-914; cH. S. Kang, J. Phys. Chem. A 2005, 109, 9292-9298; dS. Ilango, B. Vidjayacoumar, S. Gambarotta, S. I. Gorelsky, Inorg. Chem. 2008, 47, 3265-3273; eH. Wang, Z. Sun, Y. Xie, R. B. King, H. F. Schaefer III, Inorg. Chem. 2011, 50, 9256-9265; fH. Wang, H. Wang, R. B. King, H. F. Schaefer, J. Comput. Chem. 2016, 37, 250-260; gF. R. Wagner, A. I. Baranov, Y. Grin, M. Kohout, Z. Anorg. Allg. Chem. 2013, 639, 2025-2035; hS. G. Kozlova, M. R. Ryzhikov, V. A. Slepkov, S. P. Gabuda, Polyhedron 2014, 70, 59-64.

[65] aL. J. Farrugia, P. Macchi, in Electron Density and Chemical Bonding I, Springer, 2010, pp. 127-158; bL.-C. Wu, C.-W. Hsu, Y.-C. Chuang, G.-H. Lee, Y.-C. Tsai, Y. Wang, J. Phys. Chem. A 2011, 115, 12602-12615; cF.-Y. Li, L. Chen, C.-Y. Mou, S.-M. Peng, Y. Wang, Int. J. Mol. Sci. 2002, 3, 38-55; dG. La Macchia, G. Li Manni, T. Todorova, M. Brynda, F. Aquilante, B. Roos, L. Gagliardi, Inorg. Chem. 2010, 49, 5216-5222; eK. A. Kreisel, G. P. Yap, O. Dmitrenko, C. R. Landis, K. H. Theopold, J. Am. Chem. Soc. 2007, 129, 14162-14163; fG. La Macchia, F. Aquilante, V. Veryazov, B. O. Roos, L. 
Gagliardi, Inorg. Chem. 2008, 47, 11455-11457; gG. La Macchia, PhD thesis, Geneva University 2010; hA. Noor, G. Glatz, R. Müller, M. Kaupp, S. Demeshko, R. Kempe, Nat. Chem. 2009, 1, 322-325; iH. Wang, D. Die, H. Wang, Y. Xie, R. B. King, H. F. Schaefer, Theor. Chem. Acc. 2014, 133, 1459; jY. L. Huang, D. Y. Lu, H. C. Yu, J. S. K. Yu, C. W. Hsu, T. S. Kuo, G. H. Lee, Y. Wang, Y. C. Tsai, Angew. Chem. Int. Ed. 2012, 51, 7781-7785; kA. Mitschler, B. Rees, R. Wiest, M. Benard, J. Am. Chem. Soc. 1982, 104, 7501-7509; IB. O. Roos, A. C. Borin, L. Gagliardi, Angew. Chem. 2007, 119, 1491-1494; mY. I. Kurokawa, Y. Nakao, S. Sakaki, J. Phys. Chem. A 2009, 113, 3202-3209; nF. Ferrante, L. Gagliardi, B. E. Bursten, A. P. Sattelberger, Inorg. Chem. 2005, 44, 8476-8480; oR. J. Eisenhart, R. K. Carlson, K. M. Boyle, L. Gagliardi, C. C. Lu, Inorg. Chim. Acta 2015, 424, 336-344; pP. Atha, I. Hillier, M. Guest, Mol. Phys. 1982, 46, 437-448; qP. Macchi, D. Donghi, A. Sironi, J. Am. Chem. Soc. 2005, 127, 16494-16504; rL. Gagliardi, in The Chemical Bond: Chemical Bonding Across the Periodic Table, Wiley-VCH Verlag GmbH \& Co. KGaA, 2014, pp. 253-268; sR. J. Eisenhart, P. A. Rudd, N. Planas, D. W. Boyce, R. K. Carlson, W. B. Tolman, E. Bill, L. Gagliardi, C. C. Lu, Inorg. Chem. 2015, 54, 7579-7592; tM. Brynda, L. Gagliardi, P. O. Widmark, P. P. Power, B. O. Roos, Angew. Chem. Int. Ed. 2006, 45, 3804-3807; uA. Noor, T. Bauer, T. K. Todorova, B. Weber, L. Gagliardi, R. Kempe, Chem. Eur. J. 2013, 19, 9825-9832; vG. Merino, K. J. Donald, J. S. D'Acchioli, R. Hoffmann, J. Am. Chem. Soc. 2007, 129, 15295-15302; wN. Bouchakri, PhD thesis, Universite de Constantine 1 2014; xZ. Sun, H. F. Schaefer III, Y. Xie, Y. Liu, R. Zhong, Mol. Phys. 2013, 111, 2523-2535; yM. Brynda, L. Gagliardi, B. O. Roos, Chem. Phys. Lett. 2009, 471, 1-10; zG. Li Manni, A. L. Dzubak, A. Mulla, D. W. Brogden, J. F. Berry, L. Gagliardi, Chem. Eur. J. 2012, 18, 1737-1749; aaK. Albahily, V. Fomitcheva, S. Gambarotta, I. Korobkov, M. Murugesu, S. I. Gorelsky, J. Am. Chem. Soc. 2011, 133, 6380-6387; abC. Schwarzmaier, PhD thesis, Universität Regensburg 2014; acS. Gong, Q. Luo, X. Feng, Q.-s. Li, Y. Xie, R. B. King, H. F. Schaefer III, PCCP 2015, 17, 20100-20113.

[66] aL. Dahl, R. Rundle, Acta Cryst. 1963, 16, 419-426; bH. Li, H. Feng, W. Sun, Q. Fan, Y. Xie, R. B. King, H. F. Schaefer III, Mol. Phys. 2012, 110, 1637-1650; cX. Chen, Q. Du, R. Jin, H. Feng, Y. Xie, R. Bruce King, New J. Chem. 2011, 35, 1117-1127; dT. Gribanova, O. Gapurenko, R. Minyaev, V. Minkin, Russ. Chem. Bull. 2015, 64, 540-550; eL. Xu, Q.-s. Li, Y. Xie, R. B. King, H. F. Schaefer, Inorg. Chim. Acta 2010, 363, 3538-3549; fL. Fohlmeister, S. Liu, C. Schulten, B. Moubaraki, A. Stasch, J. D. Cashion, K. S. Murray, L. Gagliardi, C. Jones, Angew. Chem. Int. Ed. 2012, 51, 8294-8298; gZ. Sun, H. Wang, Y. Xie, R. B. King, H. F. Schaefer Iii, Dalton Trans. 2010, 39, 10702-10711.

[67] aC. M. Zall, D. Zherebetskyy, A. L. Dzubak, E. Bill, L. Gagliardi, C. C. Lu, Inorg. Chem. 2011, 51, 728-736; bG. La Macchia, L. Gagliardi, P. P. Power, M. Brynda, J. Am. Chem. Soc. 2008, 130, 51045114; cC. Gatti, Phys. Scr. 2013, 87, 048102; dR. Ponec, G. Lendvay, J. Chaves, J. Comput. Chem. 2008, 29, 1387-1398; eH. Wang, Y. Xie, R. B. King, H. F. Schaefer, J. Am. Chem. Soc. 2006, 128, 11376-11384; fS. Gong, C. Wang, Q.-S. Li, Y. Xie, R. B. King, J. Coord. Chem. 2012, 65, 2459-2477; gJ. Deng, Q.-s. Li, Y. Xie, R. B. King, H. Schaefer III, New J. Chem. 2013, 37, 2902-2910; hX. Gao, S. Gong, N. Li, R. B. King, New J. Chem. 2015, 39, 4939-4947; iL. Zhou, G. Li, Q.-S. Li, Y. Xie, R. B. King, Can. J. Chem. 2014, 92, 750-757; jS. T. Liddle, Molecular Metal-Metal Bonds: Compounds, Synthesis, Properties, Wiley, 2015; kY. Zeng, S. Wang, H. Feng, Y. Xie, R. B. King, Int. J. Mol. Sci. 2011, 12, 2216-2231; IH. Lei, J.-D. Guo, J. C. Fettinger, S. Nagase, P. P. Power, J. Am. Chem. Soc. 2010, 132, 17399-17401; mG. Li, L. Liu, J. Wang, Q.-s. Li, Y. Xie, R. B. King, Transition Met. Chem. 2013, 38, 617-625; nL. Bertini, C. Greco, L. De Gioia, P. Fantucci, J. Phys. Chem. A 2009, 113, 56575670; oA. D. Phillips, A. Ienco, J. Reinhold, H. C. Böttcher, C. Mealli, Chem. Eur. J. 2006, 12, 46914701; pJ. Zhang, J. Liu, Russ. J. Phys. Chem. A 2016, 90, 2564-2570; qP. Mori-Sánchez, A. Costales, M. Blanco, A. M. Pendás, V. Luaña, Departamento de Química Física y Analítica. Universidad de Oviedo, 1999; rJ. C. Silva, H. A. De Abreu, H. A. Duarte, RSC Advances 2015, 5, 2013-2023; sC. E. Hoyer, G. L. Manni, D. G. Truhlar, L. Gagliardi, J. Chem. Phys. 2014, 141, 204309.

[68] aN. K. Shee, R. Verma, D. Kumar, D. Datta, Comput. Theor. Chem. 2015, 1061, 1-5; bC.-N. Lin, W.-T. Jhu, M. Shieh, Chem. Commun. 2014, 50, 1134-1136; cS. Torelli, M. Orio, J. Pécaut, H. Jamet, L. Le Pape, S. Ménage, Angew. Chem. 2010, 122, 8425-8428; dJ. Zhou, W.-N. Wang, K.-N. Fan, Chem. Phys. Lett. 2006, 424, 247-251; eS. I. Gorelsky, X. Xie, Y. Chen, J. A. Fee, E. I. Solomon, J. Am. Chem. Soc. 2006, 128, 16452-16453; fJ. M. Goodwin, P.-C. Chiang, M. Brynda, K. Penkina, M. M. Olmstead, T. E. Patten, Dalton Trans. 2007, 3086-3092; gF. Lu, X. Li, Y. Zeng, X. Zhang, L. Meng, New J. Chem. 2014, 38, 5786-5792; hS. Pan, R. Saha, S. Mandal, P. K. Chattaraj, PCCP 2016, 18, 11661-11676.

[69] aM. Tayebani, K. Feghali, S. Gambarotta, G. P. Yap, Inorg. Chem. 2001, 40, 1399-1401; bR. Llusar, A. Beltrán, J. Andrés, F. Fuster, B. Silvi, J. Phys. Chem. A 2001, 105, 9460-9466; cL. Tang, Q. Luo, Q.s. Li, Y. Xie, R. B. King, H. F. Schaefer III, J. Chem. Theory Comput. 2012, 8, 862-874; dX. Feng, Y. Xie, R. B. King, J. Organomet. Chem. 2011, 696, 2702-2710; eS.-J. Lu, H.-G. Xu, X.-L. Xu, W.-J. Zheng, J. Phys. Chem. C 2017, 121, 11851-11861; fD.-S. Yang, M. Z. Zgierski, A. Bérces, P. A. Hackett, A. Martinez, D. R. Salahub, Chem. Phys. Lett. 1997, 277, 71-78. 
[70] aM. A. Alvarez, D. García-Vivó, M. E. García, M. E. Martínez, A. Ramos, M. A. Ruiz, Organometallics 2008, 27, 1973-1975; bM. A. Alvarez, M. E. García, D. García-Vivó, S. Menéndez, M. A. Ruiz, Organometallics 2012, 32, 218-231; cR. Ponec, G. Yuzhakov, R. Carbó-Dorca, J. Comput. Chem. 2003, 24, 1829-1838; dS. C. Liu, W. L. Ke, J. S. K. Yu, T. S. Kuo, Y. C. Tsai, Angew. Chem. 2012, 124, 6500-6503; eI. Y. Chang, J. S. K. Yu, Chem. Eur. J. 2012, 18, 9189-9193; fY. Chen, S. Sakaki, Dalton Trans. 2014, 43, 11478-11492; gH. Orita, K. Uchida, N. Itoh, J. Mol. Catal. A: Chem. 2003, 195, 173-180; hM. Carrasco, N. Curado, C. Maya, R. Peloso, A. Rodríguez, E. Ruiz, S. Alvarez, E. Carmona, Angew. Chem. Int. Ed. 2013, 52, 3227-3231; iM.-H. Baik, R. A. Friesner, G. Parkin, Polyhedron 2004, 23, 2879-2900; jY. Zhao, Y. Xue, W. Xu, J. H. Su, B. Wu, X. J. Yang, Eur. J. Inorg. Chem. 2016, 2016, 5411-5417; kA. R. Mountain, N. Kaltsoyannis, Dalton Trans. 2013, 42, 13477-13486; IN. Takagi, A. Krapp, G. Frenking, Inorg. Chem. 2011, 50, 819-826; mW. Zou, Y. Liu, W. Liu, T. Wang, J. E. Boggs, J. Phys. Chem. A 2009, 114, 646-651; nY. Zhao, X. Feng, Y. Xie, R. B. King, H. Schaefer III, J. Phys. Chem. A 2012, 116, 5698-5706; oM. C. Neary, G. Parkin, Inorg. Chem. 2017, 56, 1511-1523; pB. Mondal, M. Bhattacharyya, B. Varghese, S. Ghosh, Dalton Trans. 2016, 45, 1099911007; qP. Petrović, J. P. Djukic, A. Hansen, C. Bannwarth, S. Grimme, A. Maharromov, K. Mahmudov, M. Kopylovich, Non-covalent stabilization in transition metal coordination and organometallic complexes, Wiley-VCH: Weinheim, Germany, 2016; rK. K. Chakrahari, R. Ramalakshmi, D. Sharmila, S. Ghosh, J. Chem. Sci. 2014, 126, 1597-1603; sX. Xu, Q. Peng, M.-X. Xie, Chin. J. Struct. Chem. 2012, 31, 1287-1294; tM. R. Haque, S. Ghosh, G. Hogarth, M. G. Richmond, S. E. Kabir, Inorg. Chim. Acta 2015, 434, 150-157; uY. Nishimoto, PhD thesis, 2015; vL. Jonasson, PhD thesis, UCL (University College London) 2009; wJ. Andrés, V. S. Safont, L. Gracia, R. Llusar, E. Longo, in Recent Advances in Complex Functional Materials: From Design to Application (Eds.: E. Longo, F. d. A. La Porta), Springer International Publishing, Cham, 2017, pp. 427-454; xS. K. Bose, K. Geetharani, S. Sahoo, K. H. K. Reddy, B. Varghese, E. D. Jemmis, S. Ghosh, Inorg. Chem. 2011, 50, 9414-9422.

[71] aL. J. Farrugia, H. M. Senn, J. Phys. Chem. A 2011, 116, 738-746; bM. Niskanen, P. Hirva, M. Haukka, J. Mol. Model. 2012, 18, 1961-1968; cM. Niskanen, P. Hirva, M. Haukka, J. Chem. Theory Comput. 2009, 5, 1084-1090; dM. Niskanen, P. Hirva, M. Haukka, PCCP 2010, 12, 9777-9782; eE. P. Fowe, B. Therrien, G. Süss-Fink, C. Daul, Inorg. Chem. 2008, 47, 42-48; fM. Shieh, M. H. Hsu, W. S. Sheu, L. F. Jang, S. F. Lin, Y. Y. Chu, C. Y. Miu, Y. W. Lai, H. L. Liu, J. L. Her, Chem. Eur. J. 2007, 13, 6605-6616; gO. V. Sizova, A. Y. Sokolov, L. V. Skripnikov, V. I. Baranovski, Polyhedron 2007, 26, 4680-4690.

[72] M. A. Alvarez, M. E. García, D. Garcia-Vivo, S. Melon, M. A. Ruiz, A. Toyos, Inorg. Chem. 2014, 53, 4739-4750.

[73] aF. Poineau, L. Gagliardi, P. M. Forster, A. P. Sattelberger, K. R. Czerwinski, Dalton Trans. 2009, 5954-5959; bL. Gagliardi, B. O. Roos, Inorg. Chem. 2003, 42, 1599-1603; cR. Ponec, L. s. Bučinský, C. Gatti, J. Chem. Theory Comput. 2010, 6, 3113-3121; dX. Gao, N. Li, R. B. King, H. F. Schaefer, J. Mol. Model. 2015, 21, 7; eR. D. Adams, Y. O. Wong, Q. Zhang, Organometallics 2013, 32, 7540-7546; fY. O. Wong, 2014; gM. Costas, J. M. Poblet, M.-M. Rohmer, M. Benard, Inorg. Chem. 1995, 34, 176-183; hB. Xu, Q.-S. Li, Y. Xie, R. B. King, J. Mol. Model. 2016, 22, 157.

[74] aS. Khan, S. Pal, N. Kathewad, I. Purushothaman, S. De, P. Parameswaran, Chem. Commun. 2016, 52, 3880-3882; bD. I. Bezuidenhout, B. van der Westhuizen, A. J. Rosenthal, M. Wörle, D. C. Liles, I. Fernández, Dalton Trans. 2014, 43, 398-401; cX.-R. You, W.-J. Tian, D.-Z. Li, Y.-J. Wang, R. Li, L.-Y. Feng, H.-J. Zhai, PCCP 2016, 18, 13423-13431; dD. Bhattacharjee, B. K. Mishra, A. K. Chakrabartty, R. C. Deka, Comput. Theor. Chem. 2014, 1034, 61-72; eL. Belpassi, F. Tarantelli, A. Sgamellotti, H. M. Quiney, J. Phys. Chem. A 2006, 110, 4543-4554; fX. Li, D. Zhang, Eur. Phys. J. D 2013, 67; gD. Bhattacharjee, B. K. Mishra, R. C. Deka, J. Mater. Sci. - Mater. Electron. 2015, 50, 4586-4599; hK. L. Vikse, A. Zavras, T. H. Thomas, A. Ariafard, G. N. Khairallah, A. J. Canty, B. F. Yates, R. A. O'Hair, Organometallics 2015, 34, 3255-3263; iD.-Z. Li, S.-D. Li, J. Cluster Sci. 2011, 22, 331-341.

[75] aD.-M. Su, X.-J. Zheng, G. Schreckenbach, Q.-J. Pan, Organometallics 2015, 34, 5225-5232; bB. O. Roos, L. Gagliardi, Inorg. Chem. 2006, 45, 803-807; cG. La Macchia, M. Brynda, L. Gagliardi, Angew. Chem. Int. Ed. 2006, 45, 6210-6213.

[76] T. Yoshimura, K. Umakoshi, Y. Sasaki, A. G. Sykes, Inorg. Chem. 1999, 38, 5557-5564.

[77] N. Naumov, E. Ostanina, A. Virovets, M. Schmidtman, A. Müller, V. Fedorov, Russ. Chem. Bull. 2002, 51, 866-871.

[78] W. Bronger, T. Schuster, Z. Anorg. Allg. Chem. 1990, 587, 74-79.

[79] J. R. Long, L. S. McCarty, R. Holm, J. Am. Chem. Soc. 1996, 118, 4603-4616.

[80] M. W. Willer, J. R. Long, C. C. McLauchlan, R. Holm, Inorg. Chem. 1998, 37, 328-333.

[81] L. G. Beauvais, M. P. Shores, J. R. Long, Chem. Mater. 1998, 10, 3783-3786.

[82] S. A. Baudron, A. Deluzet, K. Boubekeur, P. Batail, Chem. Commun. 2002, 2124-2125. 
[83] T. Yoshimura, C. Suo, K. Tsuge, S. Ishizaka, K. Nozaki, Y. Sasaki, N. Kitamura, A. Shinohara, Inorg. Chem. 2010, 49, 531-540.

[84] W. Bronger, C. Koppe, D. Schmitz, Z. Anorg. Allg. Chem. 1997, 623, 239-242.

[85] Z. Zheng, J. R. Long, R. Holm, J. Am. Chem. Soc. 1997, 119, 2163-2171.

[86] T. G. Gray, R. Holm, Inorg. Chem. 2002, 41, 4211-4216.

[87] P. J. Orto, G. S. Nichol, N. Okumura, D. H. Evans, R. Arratia-Pérez, R. Ramirez-Tagle, R. Wang, Z. Zheng, Dalton Trans. 2008, 4247-4253.

[88] F. Klaiber, W. Petter, F. Hulliger, J. Solid State Chem. 1983, 46, 112-120.

[89] Y. Kim, S.-M. Park, W. Nam, S.-J. Kim, Chem. Commun. 2001, 1470-1471.

[90] M. Pyziak, J. Pyziak, M. Hoffmann, M. Kubicki, Crystal Growth \& Design 2015, $15,5223-5232$.

[91] M. Pizzonero, L. Keller, F. Dumas, M. Ourevitch, G. Morgant, A. Spasojević-de Biré, G. Bogdanović, N. E. Ghermani, J. d'Angelo, J. Org. Chem. 2004, 69, 4336-4350.

[92] N. Lugan, I. Fernandez, R. Brousses, D. A. Valyaev, G. Lavigne, N. A. Ustynyuk, Dalton Trans. 2013, 42, 898-901.

[93] A. C. Tsipis, C. E. Kefalidis, C. A. Tsipis, J. Am. Chem. Soc. 2008, 130, 9144-9155. 